\title{
Dynamics Modeling and Performance Evaluation of an Autonomous Underwater Vehicle
}

\author{
Jason P. Evans \\ Department of Mechanical Engineering \\ McGill University \\ Montreal, Quebec, Canada \\ August, 2003
}

\begin{abstract}
A thesis submitted to McGill University
in partial fulfillment of the requirements for the degree of

Master of Engineering
\end{abstract}

(C) Jason Evans

McGill University

All rights reserved. This thesis may not be reproduced in whole or in part, by photocopy or other means, without the permission of the author. 


\begin{abstract}
In recent years, autonomous underwater vehicles (AUVs) have had an increasingly pervasive role in underwater research and exploration. Development and testing of an AUV is a lengthy and costly undertaking. Not only does one risk the loss of the vehicle due to various computer errors, but also the cost of a support crew, equipment, and facilities can be prohibitively expensive. Thus, computer modeling of the vehicle is important to the AUV designer.
\end{abstract}

This thesis describes the creation of a dynamics model of an autonomous underwater vehicle. Motion equations are integrated to obtain the position and velocity of the vehicle. External forces acting on the vehicle, such as hull and control plane hydrodynamic forces, are predicted for the full $360^{\circ}$ angle of attack range. This enables the simulation of high angle of attack situations. An accurate through-body thruster model is also incorporated into the simulation. The vehicle model is validated using experimental turning diameters of the ARCS vehicle.

The model is used to simulate two configurations of the C-SCOUT AUV: the Baseline Configuration and the Fully-Actuated Configuration. The simulation is used as a predictive design tool to evaluate and compare the performance of the two configurations before they have been fully developed. Results show the Fully Actuated Configuration to be less stable than the Baseline Configuration, due to the destabilizing effect of the forward control planes. The use of the through-body thrusters on the Fully 
Actuated Configuration reduce stability if the vehicle is in motion, especially if high thruster speeds are used at low forward velocities.

Two oceanographic sampling missions and a set of controllers are devised to help determine the relative benefits of each configuration. We find that the Baseline Configuration is suitable to missions where the vehicle maintains a forward speed. The Fully Actuated Configuration is found to be suitable to missions where it can use its through-body thrusters to change direction once it has stopped. 


\section{RÉSUMÉ}

Au cours des dernières années, les véhicules sous-marins autonomes (VSMA) ont joué un rôle croissant dans l'exploration et la recherche sous-marine. Le développement et la mise à l'essai d'un VSMA forment un processus long et coûteux. En cas d'erreur informatique, les risques et pertes ne se limitent pas seulement au véhicule, mais aussi à le personnel de soutien, l'équipement et l'infrastructure. Par conséquent, la simulation virtuelle du véhicule est importante pour le concepteur d’un VSMA.

Cette thèse décrit la création d'un model dynamique d'un VSMA. Les équations de mouvement y ont été intégrées pour obtenir la position ainsi que la vélocité du véhicule. Le model permet de prédire les forces externes agissant sur le véhicule, comme la coque et la force hydrodynamique des barres de plongée couvrant les 360 degrés de l'angle d’attaque. Ceci permet de simuler des situations où l'angle d'attaque est élevé. Un model exact du propulseur-tunnel a aussi été incorporé dans cette simulation. Ce model du véhicule a été validé en utilisant les diamètres de virage expérimentaux du véhicule ARCS.

Le model est utilisé pour simuler deux configurations du VSMA C-SCOUT: la Configuration de Base et la Configuration Actionnée. Cette simulation est utilisée en tant qu'outil de conception pour évaluer et comparer la performance de deux configurations avant qu'elles n'aient été complètement développées. Les résultats montrent que la Configuration Actionnée est moins stable que la Configuration de Base, ceci étant dû à des effets déstabilisants de la barre de plongée avant. L'utilisation des 
propulseurs-tunnels dans la Configuration Actionnée réduit la stabilité du véhicule si celui-ci est en mouvement, surtout si les propulseurs sont utilisés à grande force alors que le VSMA avance à basse vélocité.

Deux missions océanographiques d'échantillonnage et un ensemble de contrôleurs ont été définis pour évaluer les avantages relatifs de chaque configuration. Nous trouvons que la Configuration de Base est appropriée pour des missions où le véhicule maintient une trajectoire vers l'avant. La Configuration Actionnée semble être appropriée pour les missions où le véhicule peut utiliser ses propulseurs-tunnels pour changer de direction une fois qu'il est arrêté. 


\section{ACKNOWLEDGements}

I would like to express my sincerest gratitude to all the people who have helped and supported me throughout my education. Thanks to the support of my friends, family, and fellow students, I have gained the determination and drive to make it through the tough times. I would especially like to thank Nyssa, who has sacrificed so much by moving halfway across the country to be with me and provided much needed love and support.

I am very grateful for the superb guidance and support of my supervisor, Professor Meyer Nahon. He was always available for advice and encouragement, and never too busy to make time for his students.

I would like to thank all those at Memorial University and the Institute for Marine Dynamics who have provided invaluable support and direction in the course of my thesis. I would especially like to thank Dr. Neil Bose and Dr. Chris Williams, who have provided excellent leadership in the C-SCOUT project.

Financial support was provided by the Natural Sciences and Engineering Research Council of Canada (NSERC) through the Strategic Research Grant entitled, 'Ocean Environmental Engineering Using Autonomous Underwater Vehicles'. 


\section{Table of Contents}

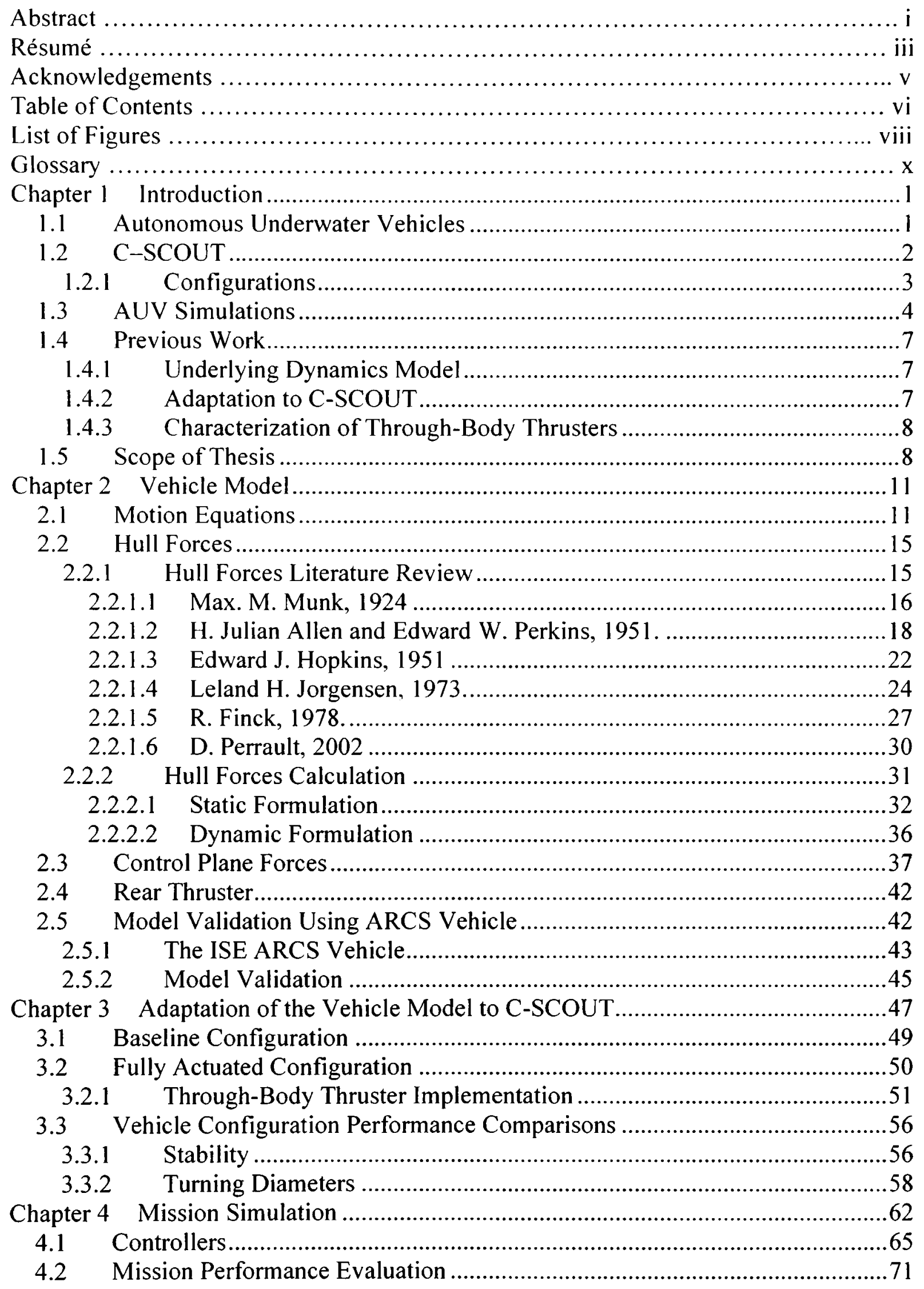


Chapter 5 Conclusions and Recommendations .............................................. 78

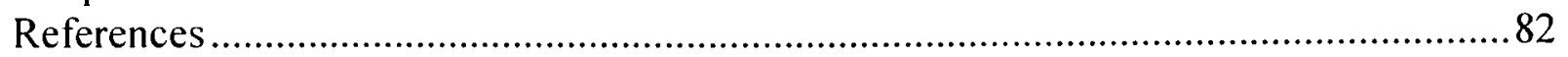

Appendix A - Derivation of Dynamic Formulation of Hull Forces..........................85 


\section{LiST OF Figures}

Figure 1.1 - AUVs Incorporating Through-Body Thrusters .....................................2

Figure 1.2 - C-SCOUT Baseline and Fully Actuated Configurations..............................4

Figure 2.1 - Vehicle Motions.............................................................................. 11

Figure 2.2 - Resultant Moment Along One of the 'Main Axis' ................................... 17

Figure 2.3 - Definition of Allen and Perkins' Polar Angle, $\theta$...................................... 19

Figure 2.4 - Illustration of Lift and Drag on Hull .................................................. 21

Figure 2.5 - Illustration of Normal and Axial Forces on Hull .....................................25

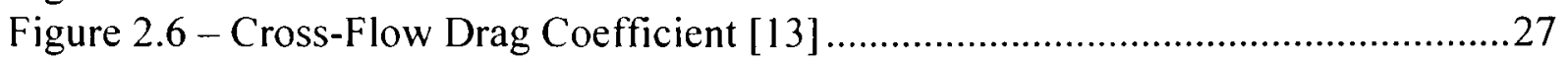

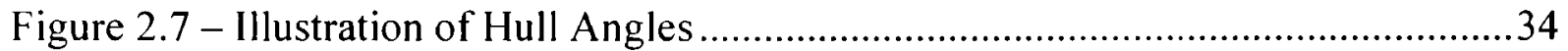

Figure 2.8 - Cross Flow Drag Proportionality Factor..................................................35

Figure 2.9 - Individual Panel on Discritized Hull ................................................... 37

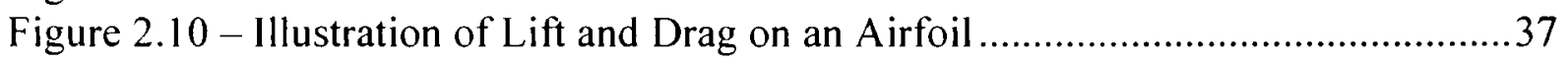

Figure 2.11 - Lift Coefficient Data Over $360^{\circ}$ Range for NACA 0012 [20] and NACA

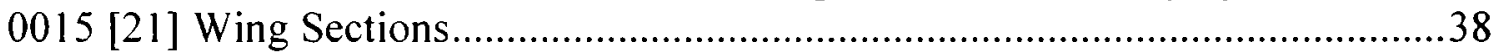

Figure 2.12 - Drag Coefficient Data Over $360^{\circ}$ Range for NACA 0012 [20] and NACA

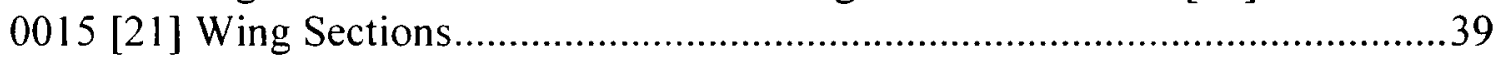

Figure 2.13 - Side View of the ISE ARCS Vehicle .............................................43

Figure 3.1 - C-SCOUT Basic Hull Drag Data...........................................................49

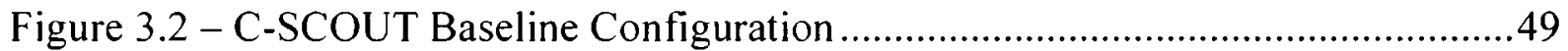

Figure 3.3 - C-SCOUT Fully Actuated Configuration .............................................50

Figure 3.4 - Tunnel Configuration................................................................... 51

Figure 3.5 - Simulink Through-Body Thruster Model [8] .......................................54

Figure 3.6 - Turning Diameters for C-SCOUT Baseline and Fully Actuated

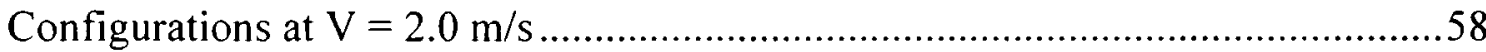

Figure 3.7 - Angle of Attack Increase on the Rear Control Planes in a Turn to the Right

(Top View) .......................................................................................59

Figure 3.8 - Turning Diameter for Fully Actuated Configuration with Front Through-

Body Thrusters at 200 RPM.................................................................61

Figure 3.9 - Turning Diameter for Fully Actuated Configuration with Front Through-

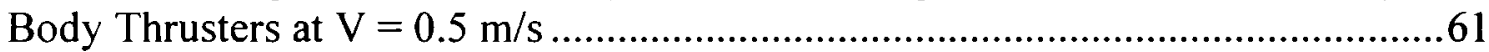

Figure 4.1 - Mission Sampling Volume and Waypoints .........................................63

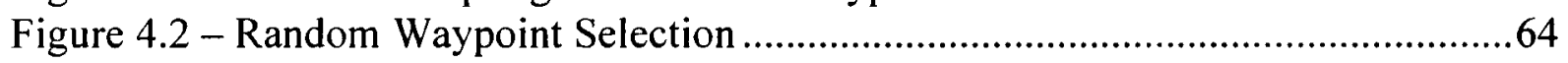

Figure 4.3 - Controller Frame Transformation Angles .............................................67

Figure 4.4 - Target Point for the Cross-Track Controller............................................67

Figure 4.5 - C-SCOUT Baseline Configuration: Continuous Sampling Mission, Inertial

X-Y-Z Plot .......................................................................................... 72

Figure 4.6 - C-SCOUT Fully Actuated Configuration: Continuous Sampling Mission,

Inertial X-Y-Z Plot ........................................................................................ 73

Figure 4.7 - C-SCOUT Baseline Configuration: Discrete Sampling Mission, Inertial X-

Y-Z Plot.

Figure 4.8 - C-SCOUT Fully Actuated Configuration: Discrete Sampling Mission,

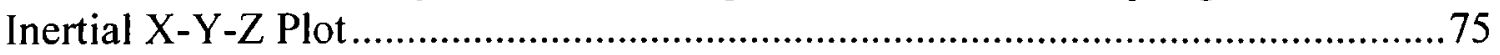

Figure 4.9 - C-SCOUT Baseline Configuration: Randomly Generated Waypoints, Inertial X-Y-Z Plot. 
Figure 4.10 - C-SCOUT Fully Actuated Configuration: Randomly Generated Waypoints, Inertial X-Y-Z Plot. 


\section{GLOSSARY}

$\begin{array}{ll}\alpha & \text { Angle of attack } \\ \alpha_{p} & \text { Angle of attack on propeller blade of through-body thruster } \\ B & \text { Sideslip angle } \\ \delta & \text { Control plane deflection } \\ \varepsilon & \text { Distance along the hull longitudinal axis from the nose } \\ \varphi & \text { Hull roll angle } \\ \varphi_{p} & \text { Pitch angle of propeller blade on through-body thruster } \\ \Phi & \text { Velocity vector angle in the body-fixed Y-Z plane } \\ \gamma & \text { Through-body thruster empirically determined added mass coefficient } \\ \eta & \text { Ratio of the drag coefficient of a circular cylinder of finite length to } \\ \psi & \text { that of a circular cylinder of infinite length } \\ \psi & \text { Yaw angle } \\ \psi_{w a x} & \text { Waypoint yaw angle error } \\ \rho & \text { Density of fluid } \\ \theta & \text { Pitch angle } \\ \theta_{p} & \text { Hydrodynamic pitch angle of through-body thruster } \\ \theta_{w a y} & \text { Waypoint pitch angle error } \\ \tau & \text { Control forces vector } \\ \omega & \text { Angular velocity vector } \\ \omega_{p} & \text { Angular speed of through-body thruster } \\ A & \text { Reference area for coefficient evaluation } \\ A_{C P} & \text { Planform area of control plane } \\ A_{D} & \text { Through-body thruster duct area } \\ A_{p} & \text { Plan-form area } \\ A_{p 0} & \text { Plan-form area from nose to station } x_{0} \\ a & \text { y-intercept of though-body thruster axial thrust/RPM graph } \\ \mathbf{a}_{\mathrm{cm}} & \text { Acceleration vector of the center of mass of the vehicle } \\ a_{e} & \text { Effective aspect ratio of the control plane } \\ b & \text { slope of though-body thruster axial thrust/RPM graph } \\ b_{C P} & \text { Control plane span } \\ B & \text { Buoyancy force } \\ C_{A} & \text { Axial coefficient } \\ C_{d} & \text { 2-D section drag coeffcient } \\ C_{D} & \text { Drag coefficient } \\ C_{D c} & \text { Drag coefficient that would be experienced by a circular cylinder } \\ & \text { section based upon the diameter and the cross component of the } \\ C_{D F} & \text { velocity } \\ \Delta C_{D F} & \text { Fore-drag coefficient } \\ C_{l} & \text { Incremental drag coefficient }\left(C_{D}-\left(C_{D}\right)_{\alpha=0}\right) \\ C_{L} & \text { 2-D section lift coefficient } \\ C_{M} & \text { Lift coefficient } \\ C_{N} & \text { Pitching-moment coefficient, in terms of reference area } A \text { and } \\ & \text { reference length } l \\ \text { Normal coefficient } \\ \end{array}$




\begin{tabular}{|c|c|}
\hline$D$ & Drag force \\
\hline$D_{p}$ & Drag force on propeller of through-body thruster \\
\hline$d$ & Body cross-section diameter \\
\hline$d_{w p}$ & Distance from the vehicle center of mass to the next waypoint \\
\hline $\mathbf{F}$ & Net force vector applied to the vehicle \\
\hline$F$ & Magnitude of normal force applied on the vehicle. \\
\hline$F_{a}$ & Axial thrust of the through-body thruster \\
\hline$F_{c l}$ & Control force along body-fixed axis $i$ \\
\hline$F_{h i}$ & Hydrodynamic force along body-fixed axis $i$ \\
\hline$F_{p}$ & Hydrodynamic resistance on the through-body thruster \\
\hline $\mathbf{F}_{\mathrm{CP}}$ & Control plane forces vector \\
\hline $\mathbf{F}_{\text {Hull }}$ & Hull forces vector \\
\hline $\mathbf{F}_{\mathrm{TT}}$ & Through-body thruster force vector \\
\hline$f_{C D}$ & $\begin{array}{l}\text { Modification ratio to transform 2-D drag coefficient to a 3-D drag } \\
\text { coefficient }\end{array}$ \\
\hline$f_{N}$ & Normal force per unit length (perpendicular to the body axis) \\
\hline$f$ & Body fineness ratio \\
\hline$g$ & Acceleration due to gravity \\
\hline h & Vector of forces acting on vehicle not including control forces \\
\hline $\mathbf{I}_{\mathrm{cm}}$ & Inertia tensor of vehicle about center of mass \\
\hline$I_{n}$ & Moments and products of inertia along body-fixed axis $i$ \\
\hline$K_{h}$ & Proportional control horizontal gain \\
\hline$K_{v}$ & Proportional control vertical gain \\
\hline$K_{a}$ & Proportional control roll gain \\
\hline$K_{T u}$ & Rear thruster velocity gain \\
\hline$K_{T x}$ & Rear thruster distance gain \\
\hline$k^{\prime}$ & $\begin{array}{l}\text { Ratio of the apparent moment of inertia to the moment of inertia of the } \\
\text { displaced air }\end{array}$ \\
\hline$k_{i}$ & Apparent mass in the $\mathrm{i}^{\text {th }}$ main axes direction \\
\hline$L$ & Lift force \\
\hline$L_{p}$ & Lift force on propeller of through-body thruster \\
\hline$l$ & Length of the body \\
\hline$l_{D}$ & Through-body thruster duct length \\
\hline$l_{0}$ & Distance from the nose to the center of mass \\
\hline$m$ & Mass of vehicle \\
\hline$M$ & Resultant moment \\
\hline $\mathbf{M}$ & Generalized mass matrix of vehicle including added mass \\
\hline $\mathbf{M}_{\mathrm{cm}}$ & Net moment vector about the vehicle's center of mass \\
\hline $\mathbf{M}_{\mathbf{C P}}$ & Moment vector about the center of mass due to the control plane forces \\
\hline $\mathbf{M}_{\text {Hull }}$ & Moment vector about the center of mass due to the hull forces \\
\hline $\mathbf{M}_{\mathrm{TT}}$ & $\begin{array}{l}\text { Moment vector about the center of mass due to the through-body } \\
\text { thrusters }\end{array}$ \\
\hline$M_{c i}$ & Control moment about axis $i$ \\
\hline$M_{h i}$ & Hydrodynamic moment about axis $i$ \\
\hline$n$ & Number of panels of the discritized hull \\
\hline$p, q, r$ & Angular velocity about the body-fixed axes \\
\hline$p_{i}, q_{i}, r_{i}$ & Angular velocity about the body-fixed axes of segment $i$ \\
\hline
\end{tabular}




\begin{tabular}{|c|c|}
\hline$\dot{\mathbf{q}}$ & $\begin{array}{l}\text { Translational and rotational velocity vector of vehicle in body-fixed } \\
\text { frame }\end{array}$ \\
\hline$q_{0}$ & Stream dynamic pressure \\
\hline$R_{D}$ & Radius of propeller of through-body thruster \\
\hline$R_{i}$ & Body radius at position $i$ \\
\hline $\mathbf{r}_{\mathrm{CE}}$ & Vector from the center of mass to the center of effort of the hull \\
\hline $\mathbf{r}_{\mathrm{CP}}$ & $\begin{array}{l}\text { Vector from the center of mass to the center of pressure of the control } \\
\text { planes }\end{array}$ \\
\hline $\mathbf{r}_{\mathrm{TT}}$ & Vector from the center of mass to the through body thruster \\
\hline$S$ & Cross-sectional area of the transverse axis of the hull \\
\hline$S_{b}$ & Area of the base of a blunt-ended body \\
\hline$S_{i}$ & Thrust reaction torque long axis $i$ \\
\hline$S_{x 0}$ & Cross-sectional area at point $x_{0}$ \\
\hline $\mathbf{T}$ & Transformation matrix from the body fixed frame to the inertial frame \\
\hline $\mathbf{T}_{\mathrm{R}}$ & $\begin{array}{l}\text { Transformation matrix from the inertial frame to the cross-track } \\
\text { controller frame }\end{array}$ \\
\hline$T_{i}$ & Thrust along body-fixed axis $i$ \\
\hline$T_{p}$ & Hydrodynamic torque on the through-body thruster \\
\hline$T_{0}$ & Equilibrium thrust \\
\hline$U_{a}$ & Fluid velocity at inlet of through-body thruster \\
\hline$U_{p}$ & Tangential propeller velocity of through-body thruster \\
\hline$u, v, w$ & Linear velocity of the center of mass in the body fixed frame \\
\hline$u_{c p}, v_{c p}, w_{c p}$ & Linear velocity of the center of pressure in the body fixed frame \\
\hline$u_{i} v_{i}, w_{i}$ & Linear velocity along the body-fixed axes of segment $i$ \\
\hline$u_{C E}, v_{C E}, w_{C E}$ & $\begin{array}{l}\text { Linear velocity of the center of effort of the hull along the body-fixed } \\
\text { axes }\end{array}$ \\
\hline$u_{T T}, v_{T T}, w_{T T}$ & Linear velocity of the through-body thruster in the body fixed frame \\
\hline & Free-stream velocity \\
\hline$V_{0}$ & Initial vehicle velocity \\
\hline$V_{B}$ & Volume of the body \\
\hline$V_{C E}$ & Total velocity at the center of effort \\
\hline$V_{c m}$ & Total velocity at the center of mass \\
\hline$V_{i}$ & Total velocity at $i$ \\
\hline$V_{R}$ & Resultant velocity on the propeller of the through-body thruster \\
\hline$X$ & Reference length for coefficient evaluation \\
\hline$X, Y, Z$ & Coordinates of the center of mass in the inertial frame \\
\hline$X_{C P}, Y_{C P}, Z_{C P}$ & Total force on the control planes in the body-fixed axes \\
\hline $\begin{array}{l}X_{\text {Hull, }}, Y_{\text {Hull }} \\
Z_{\text {Hull }}\end{array}$ & Total force on the hull body-fixed axes \\
\hline$X_{n x t}, Y_{n x t}, Z_{n x t}$ & Coordinates of next waypoint in inertial frame \\
\hline$X_{p r v}, Y_{p r v}, Z_{p r v}$ & Coordinates of previous waypoint in inertial frame \\
\hline$X_{\text {tar }}, Y_{t a r}, Z_{\text {tar }}$ & Coordinates of target position in inertial frame \\
\hline & Longitudinal distance from nose \\
\hline$x_{B}, y_{B}, z_{B}$ & Body-fixed axes \\
\hline$x_{b}, y_{b}, z_{b}$ & $\begin{array}{l}\text { Distance from the center of mass to the center of buoyancy in body- } \\
\text { fixed axes }\end{array}$ \\
\hline & Distance from the center of mass to the center of effort in the body- \\
\hline
\end{tabular}




$\begin{array}{ll}x_{C T}, y_{C T}, z_{C T} & \begin{array}{l}\text { Coordinates in the cross-track controller frame } \\ x_{p}, y_{p}, z_{p}\end{array} \\ x_{T T}, y_{T T}, z_{T T} & \begin{array}{l}\text { Distance from the center of mass to the rear thruster in body-fixed axes } \\ \mathbf{x}\end{array} \\ x_{0} & \begin{array}{l}\text { Distance from the center of mass to the through-body thruster } \\ \text { Longition and orientation vector of vehicle in inertial frame } \\ \text { used }\end{array} \\ x_{l} & \text { Longitudinal distance from nose to point at which } \frac{\mathrm{d} S}{\mathrm{~d} x} \text { has a maximum } \\ & \text { negative value } \\ x_{a c} & \text { Distance to the hydrodynamic center from the nose } \\ x_{C E} & \text { Distance to the hydrodynamic center from the center of gravity } \\ x_{m} & \text { Distance to the moment center from the nose } \\ x_{p} & \text { Distance to the centroid of plan-form area from the nose } \\ x_{p 0} & \text { Distance to the centroid of area } A p 0 \text { from the nose } \\ x_{i} & \text { Distance from the center of mass to the hull panel } \\ Y_{c}, Z_{c} & \text { Cross-drag force in the body-fixed axes } \\ Y_{l}, Z_{I} & \text { Inertia force in the body-fixed axes } \\ Y_{p}, Z_{p} & \text { Potential force in the body-fixed axes }\end{array}$




\section{Chapter 1 INTRODUCTION}

\subsection{Autonomous Underwater Vehicles}

In recent years autonomous underwater vehicles (AUVs) have had an increasingly pervasive role in underwater research and exploration. One type of vehicle that is currently expanding its roles through research and development is the Autonomous Underwater Vehicle (AUV). An AUV is an unmanned vehicle, capable of making decisions on its own without the need for a crew to monitor it. An AUV will attempt to perform a predetermined mission, based on vehicle sensor readings and intelligent response. Typical AUV missions include oceanographic surveys, environmental monitoring, iceberg profiling, bathymetric surveys, under-ice surveys, pipeline inspection, and military applications such as mine detection.

AUVs generally have a streamlined, torpedo-shaped body, and are intended for longdistance missions where the low drag enables high speeds and coverage of a large distance. Hydrodynamic fins are used to direct the vehicle and rely on forward motion to generate the forces required to change orientation. Some AUVs use a combination of 
hydrodynamic fins and through-body thrusters for control of the vehicle. Through-body thrusters enable orientation control at low speeds, while the fins provide control at higher speeds. A list of some of these AUVs includes the NPS ARIES [1], NPS PHOENIX, Proteus [2], OTTER [3], CETUS, REDERMOR [4], and C-SCOUT [5] (see Figure 1.1).

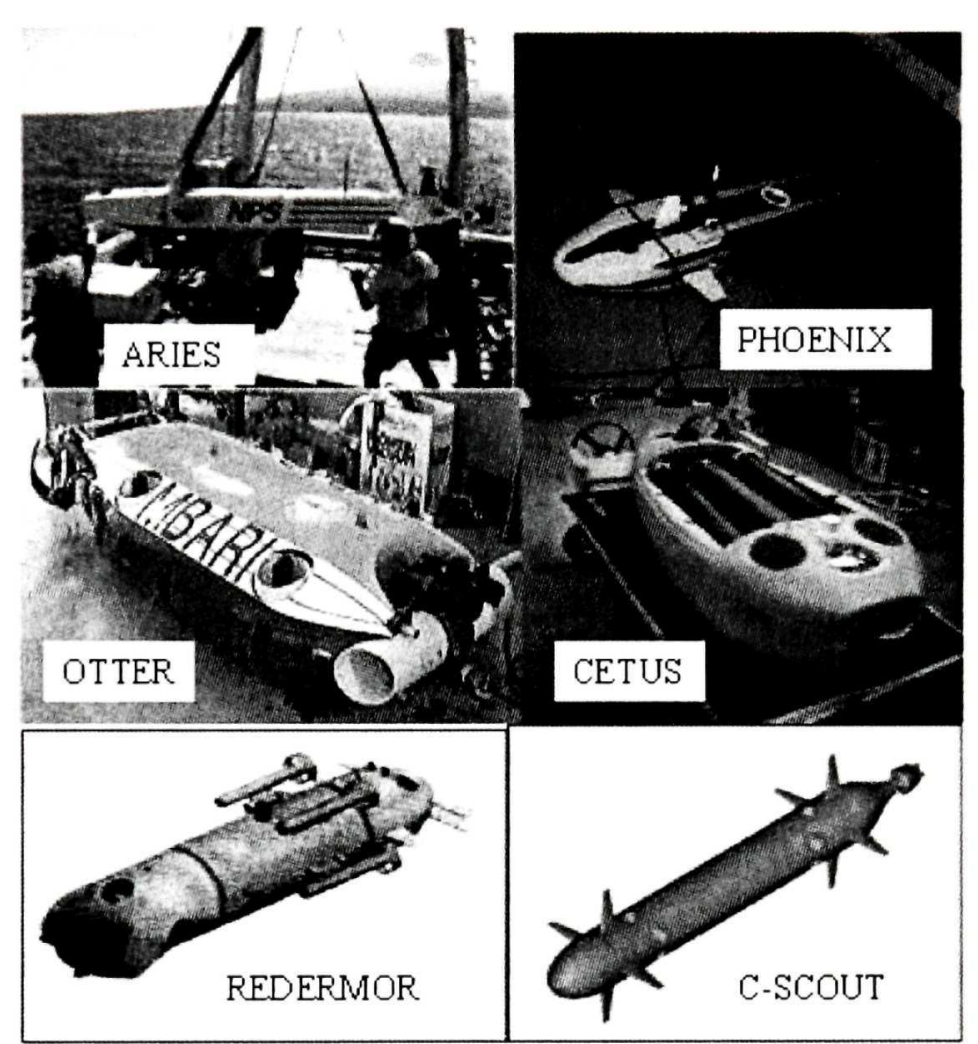

Figure 1.1 - AUVS Incorporating Through-Body Thrusters

\subsection{C-SCOUT}

C-SCOUT (Canadian Self-Contained Off-the-shelf Underwater Testbed) is an AUV currently being developed at Memorial University of Newfoundland (MUN) in St. John's, Newfoundland [5]. It is a joint project between the Institute for Marine Dynamics (IMD) and the Ocean Engineering Research Center, made possible by a 
NSERC grant entitled 'Offshore Environmental Engineering Using Autonomous Underwater Vehicles`. This grant is aimed at assessing the impact of discharges from offshore oil platforms and gas operations through the development of AUVS for environmental missions [25]. Partners in the project include IMD, NRC, C-CORE, Memorial University, University of Victoria, McGill University, Petro-Canada and the Terra-Nova Alliance, International Submarine Engineering Ltd. (ISE), and GeoResources Inc.

\subsubsection{Configurations}

C-SCOUT is designed and built with modularity in mind [5]. By incorporating multiple modules that can be interchanged, a highly configurable vehicle is obtained. These modules can contain electronics, fin actuators, sensors, ballast, through-body thrusters, and body contours. A fin module contains four fins with actuators: two are oriented horizontally and two are oriented vertically. A through-body thruster module contains three through-body thrusters: two are oriented horizontally and one is oriented vertically. In all its configurations C-SCOUT is a streamlined AUV, designed for speed and the ability to cover large distances.

The two configurations that are currently being evaluated are the Baseline Configuration (BC) and the Fully-Actuated Configuration (FAC) (see Figure 1.2). The Baseline Configuration has a rear fin module and a rear thruster for propulsion. The FullyActuated Configuration has a rear fin module, a rear through-body thruster module, a forward fin module, a forward through-body thruster module, and a rear thruster for 
propulsion. The through-body thrusters are intended to maintain maneuverability at low speeds and allow stationkeeping in the presence of a crosscurrent. Conversion from one configuration to another is nominally a simple matter of removing bolts and inserting or removing a module.

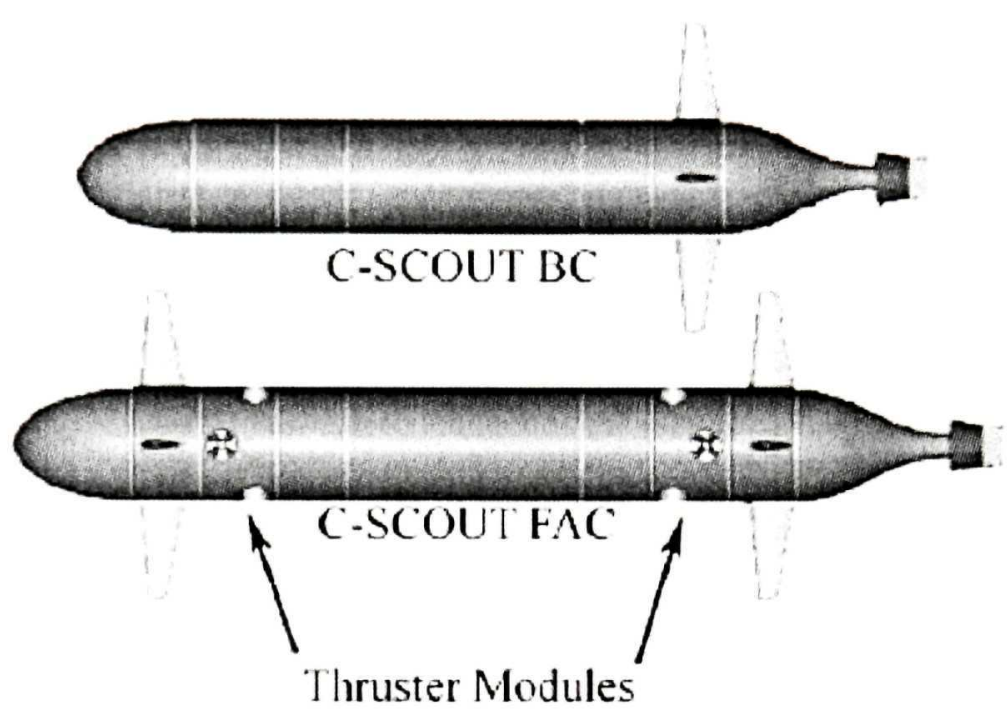

Figure 1.2 - C-SCOUT Baseline and Fully Actuated Configurations

Currently, the Baseline Configuration has been constructed and is undergoing testing at IMD and Memorial University. Thus far, the level of autonomy of C-SCOUT testing has been limited to remote control in the Ocean Engineering Basin at IMD.

\subsection{AUV Simulations}

Development and testing of an AUV is a lengthy and costly undertaking. Not only does one risk the loss of the vehicle due to various computer errors, but also the cost of a support crew, equipment, and facilities can be prohibitively expensive. Thus, computer modeling of the vehicle is important to the AUV designer. A model of the vehicle can help evaluate the effects of body profile, configuration, and environmental factors 
without risking the loss of the vehicle itself. One of the major challenges in creating such a computer model is that the vehicle dynamics must be accurately represented; otherwise we risk designing a vehicle and controllers based on erroneous information.

To fully describe the motion of an AUV, a 6 degree-of-freedom simulation is required. The dynamics of the vehicle are represented by six second-order differential equations, one for each degree of freedom of the vehicle. Once the external forces on the vehicle are determined, the accelerations in each degree of freedom can be calculated by solving the motion equations.

Hydrodynamic derivatives are commonly used to characterize the external forces on the vehicle. These are coefficients that quantify the forces on the vehicle as a function of its attitude and motion. The use of hydrodynamic derivatives in a simulation can provide very realistic results, as long as the derivatives are accurately evaluated. Hydrodynamic derivatives can be determined either by test-based or predictive methods. Test-based methods include model experiments in a wind-tunnel or tow-tank, or testing of a fullsize captive vehicle [23]. The major disadvantage of test-based methods is the need for a vehicle and the tests themselves, which can be prohibitively expensive and time consuming. Predictive methods, such as the approach presented by DATCOM [17], can yield reasonable results if the geometry of the vehicle is not too complex. Computational fluid dynamics techniques are can also be used to predict the hydrodynamic derivatives [26]. Researchers who have used hydrodynamic derivatives in their AUV simulations include Hopkin and Hertog [23], Kim et. al. [26], Encarnaca and Pascoal [27], Cristi et. al. [28], and Humphreys and Smith [32]. 
Another method used to calculate the external forces on the vehicle is the component buildup method. In this method, hydrodynamic forces are derived from empirical relations that only require specification of the vehicle geometry. Each component of the vehicle, such as the hull, control surfaces, and thrusters, is modeled separately using simple hydrodynamic relations. The forces and moments from each of these components are summed together to provide the total forces and moments acting on the hull. Use of this method enables the model to retain the vehicle's nonlinear behavior. Researchers who have used the component buildup method in their AUV simulations include Nahon [6], Perrault [7], and Prestero [29]. Additionally it is possible to use a combination of hydrodynamic derivatives and empirical relations. In their simulation, Kobayashi et. al. [30] calculate the hull forces with hydrodynamic derivatives and the control plane forces with empirical relations.

A major drawback to the simulations presented by the researchers who have implemented the component buildup method is a limitation to small angles of attack on the hull and control planes. At low velocities, or in the case of a cross current, large angles of attack can occur. For example, a vehicle performing stationkeeping in a cross current can experience an angle of attack of $90^{\circ}$ on the hull and vertical control planes. Thus we desire a simulation that can handle the full $360^{\circ}$ angle of attack range.

Our main goal is to simulate the two configurations of C-SCOUT. Therefore in order to fully model the C-SCOUT Fully Actuated Configuration, we need to include an accurate model of through-body thrusters. 


\subsection{Previous Work}

The work in this thesis concerns the dynamics modeling and control of AUVs in general, and C-SCOUT in particular. It builds on prior works performed by Nahon [6], Perrault [7], and Saunders [8].

\subsubsection{Underlying Dynamics Model}

Nahon [6] created a simplified dynamics model for streamlined underwater vehicles. $\mathrm{He}$ validated the model by creating a Matlab simulation of the ARCS vehicle, built by ISE, and compared the simulation results to measured motion of the real vehicle.

The model Nahon created decomposes the external forces acting on the vehicle into its constituent elements. These elements include weight, buoyancy, hull forces, control surface forces, and propulsion force. Summation of the component effects, along with estimated interference correction factors, determines the total forces and moments acting on the vehicle. As the model is not linearized, the nonlinear behavior of the vehicle is retained.

\subsubsection{Adaptation to C-SCOUT}

Perrault [7] adapted Nahon's simulation to model the Baseline Configuration of CSCOUT. While keeping many of the fundamental concepts contained in Nahon's model, various aspects in the simulation were altered. The simulation was also moved to the 
Simulink environment, which runs inside of Matlab. Perrault used this simulation to define vehicle behaviors based on design choices that would result in improved vehicle performance under nominal operating conditions. He also looked at acceptable vehicle behavior in the presence of control plane faults, such as jamming or loss of a control plane during operation. The design choices that were examined included hydrodynamic parameters and variations of geometry leading to added mass coefficients.

\subsubsection{Characterization of Through-Body Thrusters}

Saunders [8] focused his research on the through-body thrusters of the Fully-Actuated Configuration of C-SCOUT. Other authors had previously developed dynamic models of thrusters. However these did not include the effects of forward vehicle speed and yawed orientation on through-body thrust performance. In order to adapt the model to include these effects, an experimental system was designed and tested at Memorial University. This test setup included a tunnel thruster mounted in a full-size model of C-SCOUT, which was then towed in a tow-tank at varying speeds and orientations while the thrust output was measured. This data enabled Saunders to create a Simulink thruster model exhibiting steady-state and transient thrust behavior at varying speeds and yaw angles.

\subsection{Scope of Thesis}

The research contained herein describes the merging of Nahon, Perrault, and Saunders models to create a new underwater vehicle simulation. New formulations are incorporated to include more accurate hull force calculations and control plane 
transformations. Additionally, modifications are made to extend the validity of the model to the full $360^{\circ}$ range, which is required for accurate representation of hull and control plane forces at low speeds and in the presence of a crosscurrent.

Chapter 2 starts by describing the motion equations required for a 6 degree-of-freedom simulation of an AUV. Following this, an extensive literature review is performed to review the state of the art procedure in the estimation of hull forces on a streamlined body. From this review, a formulation is chosen based on the expected conditions the vehicle will experience during operation. This formulation is then extended from a steady-state form to a dynamic form. Also described in Chapter 2 is the estimation of the control plane forces on the vehicle while it is in motion. The simulation is adapted to the ISE ARCS vehicle and compared to experimental maneuvering data to validate the simulation.

In Chapter 3, the simulation is adapted to include the C-SCOUT Baseline and FullyActuated Configurations. Thruster models based on Saunders' work are incorporated to fully describe the Fully-Actuated Configuration. The simulation is then used to evaluate and compare the stability and turning diameters of both configurations at various vehicle speeds and control plane deflections. For the Fully-Actuated Configuration, the impact of the through-body thrusters is examined to gauge the effects on stability and turning diameter at various vehicle speeds and thruster RPM.

In Chapter 4, a series of mission plans are designed, consisting of a set of predefined waypoints. A proportional controller is designed to enable autonomous orientation 
control of the vehicle while traversing the waypoints. Three different mission plans are devised: translation along waypoints in a non-planar mower pattern, translation along the same pattern while stopping at each waypoint for stationkeeping in order to simulate a sampling technique, and translation along a randomly generated waypoint course. Additionally a set of mission tactics is developed to facilitate changing vehicle requirements as the mission progresses. The ability of both C-SCOUT configurations to successfully complete the missions is then examined.

Finally in Chapter 5, conclusions are drawn on the performance of the Baseline and Fully-Actuated configurations and their ability to complete the missions described in Chapter 4. Recommendations are also made regarding future work and possible improvements. 


\section{Chapter 2 VEHICLE MOdeL}

\subsection{Motion Equations}

The vehicle dynamics model in [6] is based on a set of dynamic equations that govern the vehicle's translational and rotational motion in 3-D space. The vehicle is considered to have 6 degrees of freedom : surge, sway, heave, roll, pitch, and yaw (see Figure 2.1).

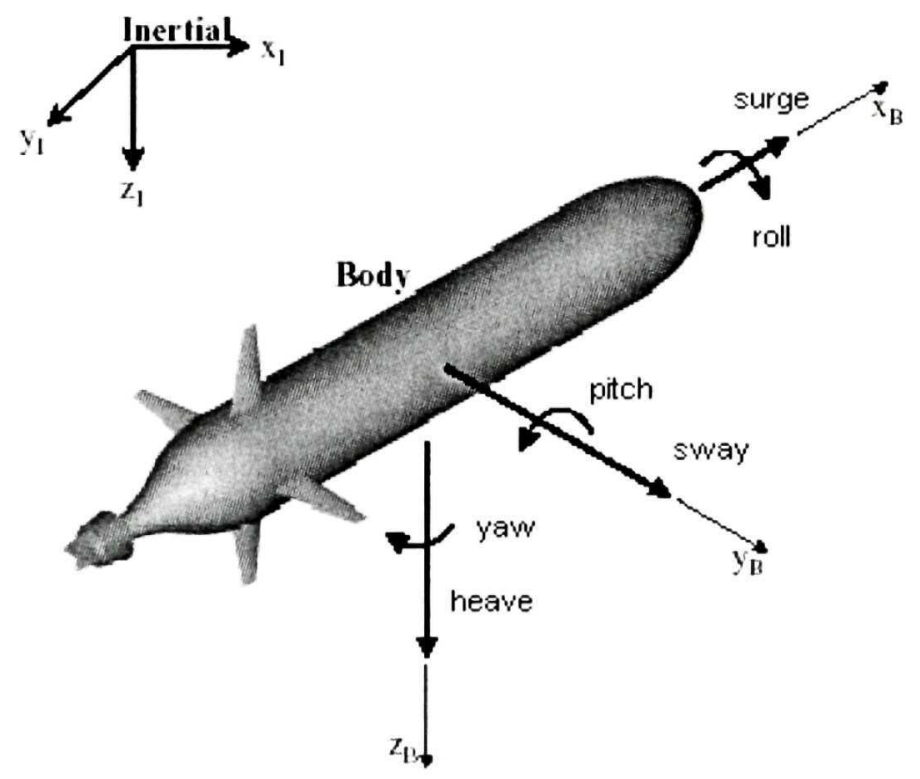

Figure 2.1 - Vehicle Motions 
The translational motions follow Newton`s law, written as:

$$
\mathbf{F}=m \mathbf{a}_{\mathrm{cm}}
$$

where $\mathbf{F}$ is the net external force applied to the vehicle, $m$ is the mass of the vehicle, and $\mathbf{a}_{\mathrm{cm}}$ is the acceleration of its mass center with respect to an inertial frame. The rotational motions follow Euler's equations:

$$
\mathbf{M}_{\mathrm{cm}}=\mathbf{I}_{\mathrm{cm}} \dot{\boldsymbol{\omega}}+\boldsymbol{\omega} \times \mathbf{I}_{\mathrm{cm}} \boldsymbol{\omega}
$$

where $\mathbf{M}_{\mathrm{cm}}$ is the net external moment acting on the vehicle at the center of mass, $\mathbf{I}_{\mathrm{cm}}$ is the inertia tensor about the vehicle`s mass center, and $\omega$ is the vehicle`s angular velocity vector.

The external forces and moments on the vehicle are due to the following effects:

- Gravitational

- Buoyancy

- Propulsive

- Control

- Hydrodynamic

In order to solve the motion equations (1) and (2), we must write them in component form. It is convenient to express them in a body-fixed coordinate system (see Figure 2.1):

$$
\begin{array}{r}
-(m g-B) \sin \theta+T_{x}+F_{c x}+F_{h x}=m(\dot{u}+q w-r v) \\
(m g-B) \sin \phi \cos \theta+T_{y}+F_{c y}+F_{h y}=m(\dot{v}+r u-p w) \\
(m g-B) \cos \phi \cos \theta+T_{z}+F_{c z}+F_{h z}=m(\dot{w}+p v-q u)
\end{array}
$$




$$
\begin{gathered}
z_{h} B \sin \phi \cos \theta+S_{v}-T_{y} z_{p}+T_{z} y_{p}+M_{c x}+M_{l l x} \\
=I_{x x} \dot{p}-\left(I_{y y}-I_{z z}\right) q r-I_{x z}(\dot{r}+p q) \\
\begin{aligned}
x_{h} B \cos \phi \cos \theta+ & z_{h} B \sin \theta+S_{y}+T_{r} z_{p}-T_{z} x_{p}+M_{c y}+M_{h y} \\
& =I_{y y} \dot{q}-\left(I_{z z}-I_{x x}\right) p r-I_{x z}\left(r^{2}-p^{2}\right) \\
-x_{h} B \sin \phi \cos \theta+ & S_{z}+T_{y} x_{p}-T_{x} y_{p}+M_{c z}+M_{l z} \\
& =I_{z z} \dot{r}-\left(I_{x x}-I_{y y}\right) p q-I_{x z}(\dot{p}-q r)
\end{aligned}
\end{gathered}
$$

where $B$ is the buoyancy force. $T_{i}, F_{C l}$, and $F_{h i}$ are the components of thrust, control, and hydrodynamic forces along axis $i$. Also $S_{i,} M_{c i}$, and $M_{h i}$ are the components of the thrust reaction torque, control, and hydrodynamic moments about axis $i . I_{x x}, I_{y y}$, and $I_{z z}$ are the vehicle's moments of inertia and $I_{x z}$ is a product of inertia. The products of inertia $I_{x y}$ and $I_{y z}$ are taken to be zero due to presumed symmetry of the vehicle about the body-fixed $x$ $z$ plane. $x_{b}$ and $z_{b}$ are the distances from the center of mass to the center of buoyancy in the body-fixed $\mathrm{X}$-axis and Z-axis, respectively. $x_{p}, y_{p}$, and $z_{p}$ are the distances from the center of mass to the rear thruster in the body-fixed $\mathrm{X}, \mathrm{Y}$, and Z-axes, respectively. The translational and rotational velocities in the body-fixed frame are $u, v, w$, and $p, q, r$ respectively. Finally $\phi, \theta$, and $\psi$ are the three Euler angles (roll, pitch, yaw) that specify the vehicle's orientation in space.

The motion equations listed in (3) and (4) can also be described in the form

$$
\ddot{\mathbf{q}}=\mathbf{M}^{-1}[\boldsymbol{\tau}-\mathbf{h}(\mathbf{x}, \dot{\mathbf{q}})]
$$

where $\mathbf{M} \in \mathbf{R}^{6.6}$ is the inertia matrix including added mass, $\mathbf{h}(\mathbf{x}, \dot{\mathbf{q}}) \in \mathbf{R}^{6}$ is a vector that includes centrifugal, Coriolis, gravitational, buoyancy, and hydrodynamic forces, and $\boldsymbol{\tau} \in \mathbf{R}^{6}$ is a vector of control forces. $\mathbf{x}$ is the position state vector in the inertial frame, 
$\dot{\mathbf{q}}$ is the velocity state vector in the body-fixed frame, and $\ddot{\mathbf{q}}$ is the acceleration vector in the body-fixed frame:

$$
\begin{aligned}
& \mathbf{x}=\left[\begin{array}{llllll}
X & Y & Z & \phi & \theta & \psi
\end{array}\right]^{\mathrm{T}} \\
& \dot{\mathbf{q}}=\left[\begin{array}{lllllll}
u & v & w & p & q & r
\end{array}\right]^{\top}
\end{aligned}
$$

The inertial velocity vector, $\dot{\mathbf{x}}$ (the derivative of the position vector), and the body-fixed velocity vector, $\dot{\mathbf{q}}$, are related as follows:

$$
\dot{\mathbf{x}}=\mathbf{T} \dot{\mathbf{q}}
$$

where $\mathbf{T}$ is the transformation matrix from the body-fixed frame to the inertial frame:

$$
\begin{gathered}
\mathbf{T}=\left[\begin{array}{cccccc}
c \psi c \theta & -s \psi c \phi+c \psi s \theta s \phi & s \psi s \phi+c \psi c \phi s \theta & 0 & 0 & 0 \\
s \psi c \theta & c \psi c \phi+s \phi s \theta s \psi & -c \psi s \phi+s \theta s \psi c \phi & 0 & 0 & 0 \\
-s \theta & c \theta s \phi & c \theta c \phi & 0 & 0 & 0 \\
0 & 0 & 0 & 1 & s \phi t \theta & c \phi t \theta \\
0 & 0 & 0 & 0 & c \phi & -s \phi \\
0 & 0 & 0 & 0 & s \phi / c \theta & c \phi / c \theta
\end{array}\right] \\
s \equiv \sin (\cdot), c \equiv \cos (\cdot), t \equiv \tan (\cdot)
\end{gathered}
$$

Therefore in order to solve the motion equations we conduct the following procedure:

1. Choose the initial states of the vehicle, $\mathbf{x}$ and $\dot{\mathbf{x}}$.

2. Transform the inertial velocity vector into the body-fixed frame using $\dot{\mathbf{q}}=\mathbf{T}^{-1} \dot{\mathbf{x}}$.

3. Evaluate the forces (hydrodynamic, buoyancy, etc.) acting on the vehicle based on the orientation and velocity.

4. Solve for the body-fixed acceleration vector, $\ddot{\mathbf{q}}$, using (5).

5. Transform the acceleration vector, $\ddot{\mathbf{q}}$, into the inertial frame using $\ddot{\mathbf{x}}=\dot{\mathbf{T}} \dot{\mathbf{q}}+\mathbf{T} \ddot{\mathbf{q}}$ 
6. Integrate the acceleration vector, $\ddot{\mathbf{x}}$, to obtain the velocity vector, $\dot{\mathbf{x}}$, in the inertial frame.

7. Integrate the velocity vector, $\dot{\mathbf{x}}$, to obtain the position vector in the inertial frame, $\mathbf{x}$.

8. Loop back to step 2 to continue.

\subsection{Hull Forces}

The hull force calculation described in Nahon's [6] simulation is not suitable for large angles of attack, and so must be improved for the present purpose. For this reason a literature review was performed on the topic of hull forces on streamlined bodies of revolution in the subsonic range at angles of attack in order to choose the best possible formulation for our application.

\subsubsection{Hull Forces Literature Review}

Various researchers have attempted to estimate the hull forces for a streamlined body of revolution at angles of attack. In 1924, Munk [9] proposed the idea that the hull force on airships can be estimated by a potential flow analysis of the flows around a streamlined body. Later other researchers, such as Allen and Perkins [10], [11], Hopkins [12], and Jorgensen [13], continued this analysis. Papers published by of these authors and others have been summarized in sections 2.2.1.1 to 2.2.1.7. Special attention has been paid to the characteristics of hull forces we are most interested in; namely the estimation of lift, drag, and pitching moment coefficients. 


\subsubsection{Max. M. Munk, 1924}

Based on potential flow theory, Munk [9] develops equations for the aerodynamic forces on airship hulls. In his analysis, the viscosity and compressibility of the fluid are neglected.

Munk defines 3 axes that he calls "main axes", which are parallel and perpendicular to the direction of motion. If the body is moving with the velocity components $u, v$, and $w$ parallel to the three main axes, a resultant moment will be generated. The exception to this is when the apparent mass is the same in all directions (such as a sphere). In practice motion will occur in a main plane at right angles to a main axis. The resultant moment in a single plane is then described as the following:

$$
M=\left(k_{2}-k_{1}\right) \frac{\rho V^{2}}{2} \sin (2 \alpha)
$$

where $M$ is the resultant moment, $k_{i}$ are the apparent mass factors in the main axis directions based on length to diameter ratios (see Table 2.1), $\rho$ is the fluid density, $V$ is the free stream velocity, and $\alpha$ is the angle of attack (see Figure 2.2). The apparent mass factor, $\left(k_{2}-k_{1}\right)$, approaches unity if the body is very elongated, or of high fineness ratio, which is defined as:

$$
f=\frac{l}{d}
$$

where $l$ is the body length, and $d$ is the body cross-sectional diameter. 


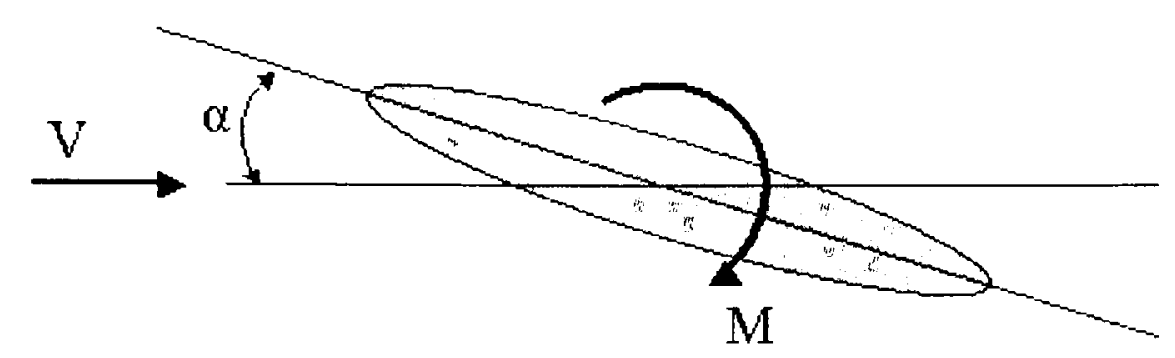

Figure 2.2-Resultant Moment Along One of the 'Main Axis'

\begin{tabular}{|c|c|c|c|c|}
\hline$f$ & $\begin{array}{c}k_{l} \\
\text { (longitudinal) }\end{array}$ & $\begin{array}{c}k_{2} \\
\text { (transverse) }\end{array}$ & $k_{2}-k_{l}$ & $\begin{array}{c}k^{\prime} \\
\text { (rotation) }\end{array}$ \\
\hline 1 & 0.500 & 0.500 & 0 & 0 \\
\hline 1.50 & 0.305 & 0.621 & 0.316 & 0.094 \\
\hline 2.00 & 0.209 & 0.702 & 0.493 & 0.240 \\
\hline 2.51 & 0.156 & 0.763 & 0.607 & 0.367 \\
\hline 2.99 & 0.122 & 0.803 & 0.681 & 0.465 \\
\hline 3.99 & 0.082 & 0.860 & 0.778 & 0.608 \\
\hline 4.99 & 0.059 & 0.895 & 0.836 & 0.701 \\
\hline 6.01 & 0.045 & 0.918 & 0.873 & 0.764 \\
\hline 6.97 & 0.036 & 0.933 & 0.897 & 0.805 \\
\hline 8.01 & 0.029 & 0.945 & 0.916 & 0.840 \\
\hline 9.02 & 0.024 & 0.954 & 0.930 & 0.865 \\
\hline 9.97 & 0.021 & 0.960 & 0.939 & 0.883 \\
\hline$\infty$ & 0.000 & 1.000 & 1.000 & 1.000 \\
\hline
\end{tabular}

Table 2.1 - Values for $k_{i}$ Provided by Munk [9]

Munk makes the statement that the motion of the air in each plane can be considered 2dimensional. i.e. for a body in translational horizontal motion at an angle of attack, the air will flow around the axis of the hull, and not only along it. In other words, the air located in a vertical plane at right angles to the motion will remain in that plane as the body passes through it. The normal force per unit length can be computed as:

$$
f_{N}=\frac{\mathrm{d} F}{\mathrm{~d} x}=\left(k_{2}-k_{1}\right) \frac{\rho V^{2}}{2} \sin (2 \alpha) \frac{\mathrm{d} S}{\mathrm{~d} x}
$$


where $f_{N}$ is the normal force per unit length, $F$ is the total normal force acting on the body, $x$ is the longitudinal distance along the hull from the nose, and $S$ is the crosssectional area of the transverse axis of the hull.

\subsubsection{H. Julian Allen and Edward W. Perkins, 1951.}

In 1951. Allen and Perkins published two important papers entitled, A Study of the Effects of Viscosity on Flow Over Slender Inclined Bodies of Revolution [10], and Characteristics of Flow Over Inclined Bodies of Revolution [11]. Allen and Perkins confirm the results of Munk's potential analysis of a streamlined surface of revolution for inviscid flow. They state that there are discrepancies between this result and experimental results due to the neglected viscosity effects. These discrepancies include the following:

- The experimental lift force exceeds the calculated lift force by an amount which increases with increasing angle of attack

- The absolute magnitude of the resultant moment is less than that calculated due to the center of pressure being further aft.

A comparison of the inviscid flow analysis with experiments showed good agreement for the forward sections of the hull around the perimeter of the hull, with the exception of $\theta$ values near $180^{\circ}$ (In their paper, $\theta$ is defined as the polar angle about the longitudinal axis measured from the approach direction of the cross-flow velocity, as shown in Figure 2.3). This discrepancy increases with increasing distance from the bow, and increases very rapidly downstream of the maximum diameter section. Allen and 
Perkins state that this discrepancy results from neglecting the viscous effects of the fluid. They conclude that the inviscid flow solution can be expected to hold over the forebody, but on the afterbody, the fluid viscosity will influence the pressure distribution, particularly on the lee side.

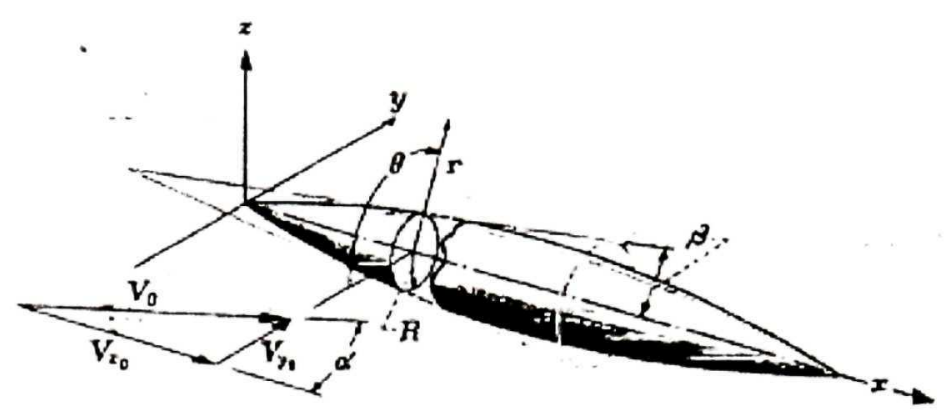

Figure 2.3 - Definition of Allen and Perkins' Polar Angle, $\theta$

Allen and Perkins make the assertion that the viscous cross-force distribution could be calculated on the assumption that each circular element along the body experiences a cross force equal to the drag force the section would experience with the axis of revolution normal to the stream moving at the velocity $V \sin \alpha$. This contribution would add the viscous term to the potential flow term. Ward [16] showed the potential cross force to be directed midway between the normal to the axis of revolution and the normal to the flow direction. This modification would add a $(\cos \alpha / 2)$ to the potential term. Thus the local normal force per unit length at station $x$ presented by Allen and Perkins is the following:

$$
f_{N}=\left(k_{2}-k_{1}\right) q_{0} \frac{\mathrm{d} S}{\mathrm{~d} x} \sin (2 \alpha) \cos \frac{\alpha}{2}+2 \eta R_{x} C_{D c} q_{0} \sin ^{2} \alpha
$$

where the apparent mass factor, $\left(k_{2}-k_{1}\right)$, is listed by Munk in Table 2.1, $\eta$ is a ratio to account for the effects of a finite length cylinder as opposed to an infinite length cylinder, $R_{x}$ is the radius at position $x, C_{D c}$ is the drag coefficient that would be 
experienced by a circular cylinder section based upon the diameter and the cross component of the velocity, and $q_{0}$ is the stream dynamic pressure:

$$
q_{11}=\frac{\rho V^{2}}{2}
$$

Equation (14) can be integrated over the entire body to find the lift coefficient, $C_{L}$, foredrag coefficient, $C_{D F}$, and the pitching moment coefficient, $C_{M}$. The lift and drag are defined as perpendicular and parallel to the incoming flow on the hull (see Figure 2.4). The pitching moment coefficient is calculated about an arbitrary position a distance $x_{m}$ from the nose.

$$
\begin{aligned}
& C_{L}=\left(k_{2}-k_{1}\right) \frac{S_{b}}{A} \sin (2 \alpha) \cos \frac{\alpha}{2}+\eta C_{D_{c}} \frac{A_{p}}{A} \sin ^{2} \alpha \cos \alpha \\
& C_{D F}=C_{D F:(\alpha=0)} \cos ^{3} \alpha+\left(k_{2}-k_{1}\right) \frac{S_{b}}{A} \sin (2 \alpha) \sin \frac{\alpha}{2}+\eta C_{D c} \frac{A_{p}}{A} \sin ^{3} \alpha \\
& C_{M}=\left(k_{2}-k_{1}\right)\left(\frac{\mathrm{V}_{B}-S_{b}\left(l-x_{i m}\right)}{A X}\right) \sin (2 \alpha) \cos \frac{\alpha}{2}+\eta C_{D c} \frac{A_{p}}{A}\left(\frac{x_{m}-x_{p}}{X}\right) \sin ^{2} \alpha
\end{aligned}
$$

where $S_{b}$ is the cross-sectional area of the base, $A$ is the reference area, $A_{p}$ is the planform area of the vehicle, $C_{D F(\alpha=0)}$ is the fore-drag coefficient at zero angle of attack, $V_{B}$ is the volume of the body, $X$ is the reference length, and $x_{p}$ is the distance from the nose to the centroid of the planform area. The bodies analyzed by Allen and Perkins are blunt-based slender bodies of revolution. Thus the base area, $S_{b}$, which appears in (17) is always non-zero for their results. This would likely lead to errors for a pointed-base body as the potential inviscid term would be zero. 


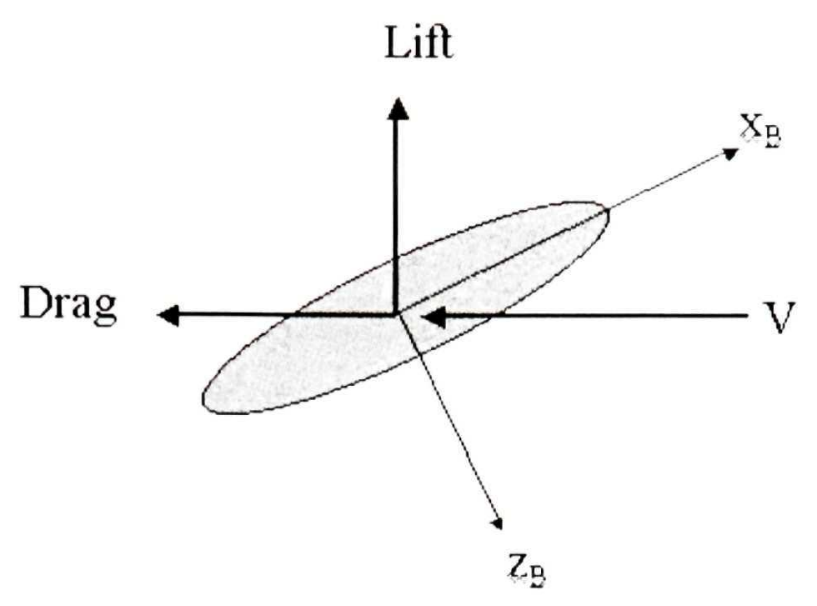

Figure 2.4-Illustration of Lift and Drag on Hull

The first term on the right side of each equation in (17) is the potential contribution, while the second term is the viscous contribution. Allen and Perkins note that these allowances for viscous effects is approximate and is only expected to apply well to bodies of high fineness ratio due to the assumed two-dimensional nature of the viscous cross flow.

Allen and Perkins use a small angle approximation on $\alpha$ to give the following:

$$
\begin{aligned}
& C_{L}=2\left(k_{2}-k_{1}\right) \frac{S_{b}}{A} \alpha+\eta C_{D c} \frac{A_{p}}{A} \alpha^{2} \\
& \Delta C_{D F}=C_{D F}-C_{D F(\alpha=0)}=\left(k_{2}-k_{1}\right) \frac{S_{b}}{A} \alpha^{2}+\eta C_{D c} \frac{A_{p}}{A} \alpha^{3} \\
& C_{M}=2\left(k_{2}-k_{1}\right)\left(\frac{\mathrm{V}_{B}-S_{b}\left(l-x_{m}\right)}{A X}\right) \alpha+\eta C_{D c} \frac{A_{p}}{A}\left(\frac{x_{m}-x_{p}}{X}\right) \alpha^{2}
\end{aligned}
$$

Allen and Perkins compare their formulations to empirical data, examining the effect of cross-flow Reynolds number, cross-flow Mach number, and pressure distribution about 
the perimeter of the body. In general the results of the viscous formulation show a much better correlation to empirical data than the potential formulation originally put forth by Munk [9]. They show that the lift and drag characteristics are fairly accurately predicted, but that the pitching moment is overestimated.

\subsubsection{Edward J. Hopkins, 1951}

In his paper, Hopkins [12] attempts to improve on the work by Allen and Perkins [11] to account for the error in the pitching moment coefficient. Hopkins states that the potential theory is accurate on the expanding portion of the body, whereas the effects of viscosity become important on the contracting portion.

Hopkins states that the experimental and theoretical results of the pitching moment coefficient from Allen and Perkins are not in agreement, as the longitudinal distribution of the transverse load is not accurately represented. This is due to the fact that both contributions are applied over the entire length. Hopkins improves this by assuming potential flow only over the expanding portion of the body and viscous flow over the remainder of the body. Thus he presents his equations for lift coefficient, drag coefficient, and pitching moment coefficient (for a small angle approximation) as follows: 


$$
\begin{aligned}
& C_{I .}=\frac{2 \alpha\left(k_{2}-k_{1}\right)^{x_{11}}}{V_{B}^{\frac{2}{3}}} \int_{0}^{\mathrm{d} S} \frac{2 \alpha^{2}}{\mathrm{~d} x} \mathrm{~d} x+\frac{\alpha^{\frac{2}{3}}}{V_{x_{11}}} \eta R_{x} C_{I k_{c}} \mathrm{~d} x \\
& \Delta C_{D}=\frac{2 \alpha^{2}\left(k_{2}-k_{1}\right)^{x_{11}}}{V_{B}^{\frac{2}{3}}} \frac{\mathrm{d} S}{\mathrm{~d} x} \mathrm{~d} x+\frac{2 \alpha^{3}}{V_{B}^{\frac{2}{3}}} \int_{x_{11}}^{1} \eta R_{x} C_{I_{c}} \mathrm{~d} x \\
& C_{M}=\frac{2 \alpha\left(k_{2}-k_{1}\right)^{x_{11}}}{V_{B}} \int_{0}^{\mathrm{d} S} \frac{2 \alpha^{2}}{\mathrm{~d} x}\left(x_{m}-x\right) \mathrm{d} x+\frac{2 V_{B}}{V_{x_{11}}} \eta R_{x} C_{D C}\left(x_{m}-x\right) \mathrm{d} x
\end{aligned}
$$

Note that $\Delta C_{D}$ is the increase in the body drag coefficient above that at an angle of attack of zero degrees. Equations (19) are similar to (18) presented by Allen and Perkins with the following exceptions:

1. The limits of integration differ. The first term in each equation in (19) is integrated only to $x_{0}$, defined in $(20)$. The second term in each equation is integrated only from $x_{0}$ to the tail end.

2. The first term in the drag coefficient equation is greater by a factor of 2 , due to a difference in the derivation. Hopkins derived his equation by considering the drag increment from potential theory to be equal to the transverse force given by Munk [9] multiplied by the angle of attack. Hopkins found that this was a good approximation by comparing the product of the experimental lift coefficient and the angle of attack (in radians) with the increment of experimental drag coefficient for the fifteen bodies of revolution he examined.

3. The reference area, $A$, is $V_{B}^{\frac{2}{3}}$, and presumably the reference length, $X$, is $V_{B}^{\frac{1}{3}}$.

Hopkins found that the longitudinal distance $x_{0}$ could be correlated with the longitudinal station along the body at which the rate of change of cross-sectional area with 
longitudinal distance has a maximum negative value. By combining this distance for 15 bodies of revolution in a line of regression, Hopkins obtained the following relationship:

$$
\frac{x_{0}}{l}=0.378+0.527 \frac{x_{1}}{l}
$$

where $x_{l}$ is the longitudinal distance from the nose to a point at which $\frac{\mathrm{d} S}{\mathrm{~d} x}$ has a maximum negative value.

For all but one of the 15 bodies of revolution tested, the pitching moment coefficient computed by Hopkins was shown to be in a closer agreement to the empirical data than that of Allen and Perkins for low angles of attack. Hopkins mentions that better agreement for higher angles of attack could have been realized if $x_{0}$ had been allowed to move forward along the body with increasing angle of attack. Hopkins' formulation of lift and drag coefficients produced good agreement with the empirical data. However he could not conclude if his method was a better correlation than that of Allen and Perkins based on the results, as both methods produced good agreement with the empirical data.

\subsubsection{Leland H. Jorgensen, 1973.}

Jorgensen's paper [13] uses Allen and Perkins' method of using both a potential and viscous term in the calculation of hull forces. In contrast to the papers previously discussed, Jorgensen creates formulas for axial and normal coefficients (see Figure 2.5), as opposed to lift and drag coefficients (see Figure 2.4). These forces act perpendicular and parallel to the direction of motion, respectively. These expressions are developed for angles of attack from $0^{\circ}$ to $180^{\circ}$ : 
$C_{N}=\frac{S_{b}}{A} \sin 2 \alpha^{\prime} \cos \frac{\alpha^{\prime}}{2}+\eta C_{D C} \frac{A_{P}}{A} \sin ^{2} \alpha^{\prime}$

$0^{\circ} \leq \alpha \leq 180^{\circ}$

$C_{A}=C_{D I F\left(\alpha=0^{\circ}\right)} \cos ^{2} \alpha^{\prime}$

$0^{\circ} \leq \alpha \leq 90^{\circ}$

$C_{A}=C_{D H \cdot\left(\alpha=180^{\circ}\right)} \cos ^{2} \alpha^{\prime}$

$90^{\circ} \leq \alpha \leq 180^{\circ}$

$C_{M}=\left[\frac{V_{B}-S_{b}\left(l-x_{m}\right)}{A d}\right] \sin 2 \alpha^{\prime} \cos \frac{\alpha^{\prime}}{2}+\eta C_{D C} \frac{A_{p}}{A}\left(\frac{x_{m}-x_{p}}{d}\right) \sin ^{2} \alpha^{\prime} \quad 0^{\circ} \leq \alpha \leq 90^{\circ}$

$C_{M}=-\left[\frac{V_{B}-S_{b} x_{m}}{A d}\right] \sin 2 \alpha^{\prime} \cos \frac{\alpha^{\prime}}{2}+\eta C_{D c} \frac{A_{p}}{A}\left(\frac{x_{m}-x_{p}}{d}\right) \sin ^{2} \alpha^{\prime} \quad 90^{\circ} \leq \alpha \leq 180^{\circ}$

where $C_{N}$ is the normal force coefficient and $C_{A}$ is the axial force coefficient.

also

$$
\begin{array}{lll}
\alpha^{\prime}=\alpha & \text { for } & 0^{\circ} \leq \alpha \leq 90^{\circ} \\
\alpha^{\prime}=180^{\circ}-\alpha & \text { for } & 90^{\circ} \leq \alpha \leq 180^{\circ}
\end{array}
$$

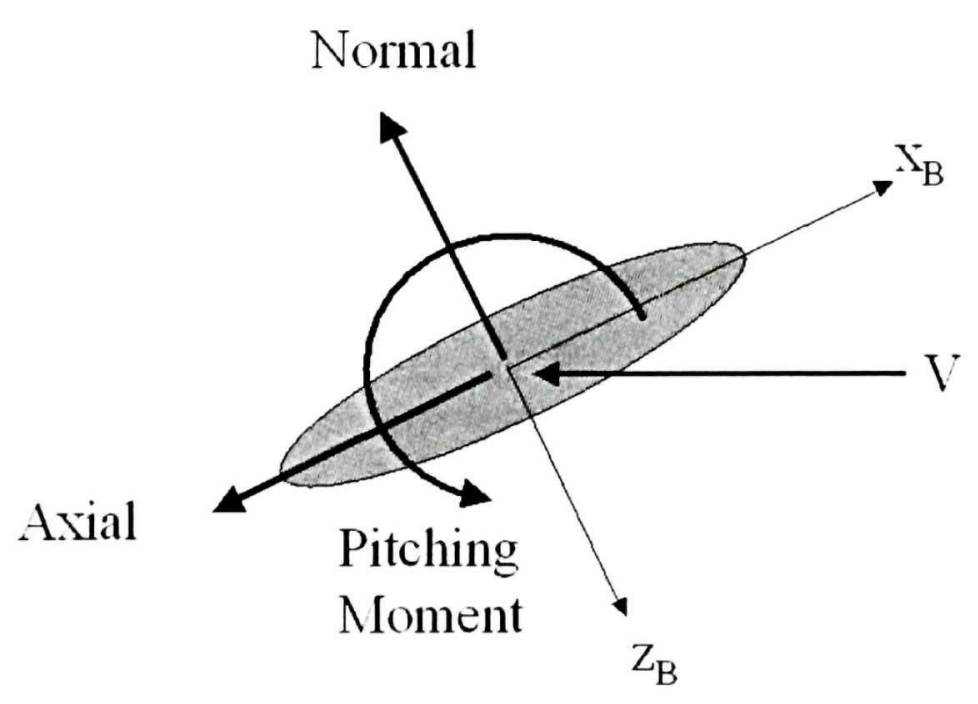

Figure 2.5 - Illustration of Normal and Axial Forces on Hull 
The aerodynamic center measured from the nose, $x_{a c}$, is given by

$$
x_{a c}=\left(\frac{x_{m}}{d}-\frac{C_{M I}}{C_{N}}\right) d
$$

Jorgensen does not include the apparent mass factor in the potential terms. This will have a small effect on high fineness ratio bodies, as it will be about equal to 1 . The bodies used for experimental data all have a blunt base, and as such the cross-sectional area of the base is not zero for any of Jorgensen's test cases. The reference area used is cross-sectional area of the cylindrical part of the body.

Jorgensen compares his results with 9 different bodies of revolution of high fineness ratio at a Mach number of 2.86 . With the exception of the axial coefficient, Jorgensen quotes good agreement with the data throughout the angle of attack range of $0^{\circ}$ to $180^{\circ}$. Unfortunately he does not perform these tests at subsonic speeds (incompressible flow range), which would be our primary interest. Using his equations, Jorgensen examines the effects of Reynolds number on the normal coefficient and the aerodynamic center position. His results show that the normal coefficient and aerodynamic center vary considerably with changing cross-flow Reynolds number, due to the strong influence of the varying cross-flow drag coefficient. There is a considerable drop in the cross-flow drag coefficient from Reynolds numbers of $10^{5}$ to $10^{6}$, followed by a gradual rise to $10^{7}$ (see Figure 2.6), which will in turn affect the computed normal coefficient and aerodynamic center position. 


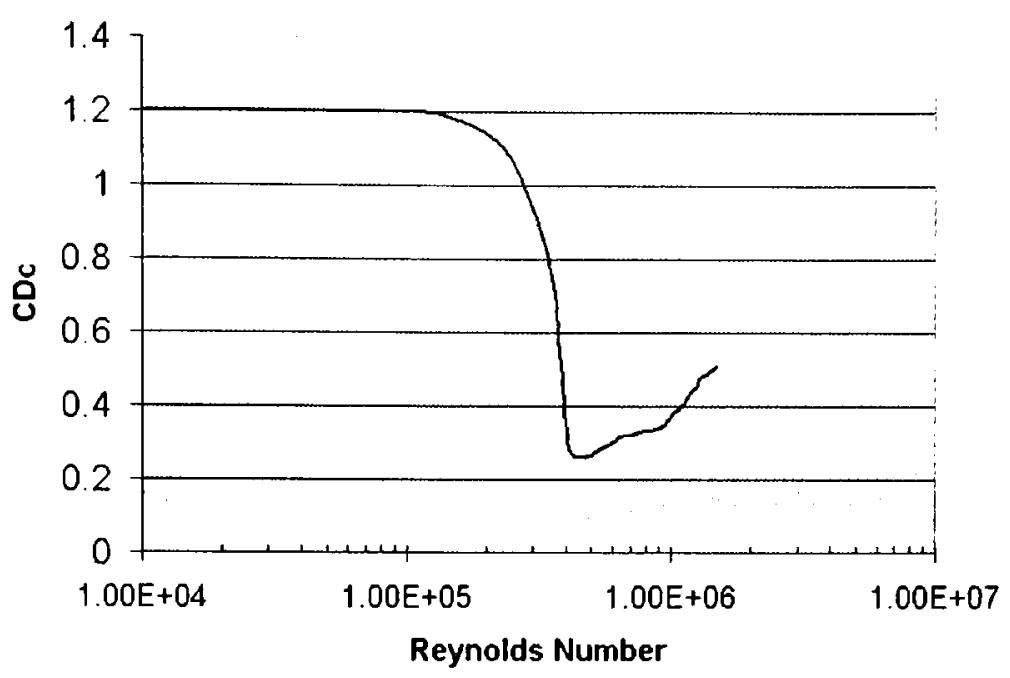

Figure 2.6-Cross-Flow Drag Coefficient [13]

\subsubsection{R. Finck, 1978 .}

USAF Stability and Control DATCOM [17] presents methods for estimating lift, drag, normal, axial, and pitching-moment coefficients for various shapes at small and large angles of attack. These methods are based on research by Munk [9], Allen and Perkins [10] [11], Hopkins [12], Jorgensen [13], as well as others that are not examined in this thesis, such as Polhamus [14] and Kelly [15]. DATCOM suggests which method to use based on the amount of substantiated experimental data for each method and circumstances specific to the application, such as angle of attack and body geometry.

For small angles of attack, DATCOM presents methods that employ only the potential term in the equations calculating the lift, drag, normal, axial, and pitching-moment coefficients. Since we are primarily interested in higher angles of attack, we will examine DATCOM's approaches for higher angles of attack, which consider the viscous effects. 


\section{Lift and Normal Coefficients:}

For body lift in the nonlinear angle of attack range DATCOM presents 3 methods:

1. The method of Hopkins [12] for calculating the lift coefficient, which was discussed in Section 2.2.1.3.

2. A method by E. Polhamus [14] for calculating the lift coefficient, modified for application of thick bodies. This method is based on the concept of vortex lift for sharp delta wings.

3. The method by Jorgensen [13] for calculating the normal force coefficient, which was discussed in Section 2.2.1.4.

DATCOM states that method 1 is satisfactory for angles of attack up to about $12^{\circ}$, as a small angle approximation has been made, and method 2 is satisfactory for angles of attack up the about $20^{\circ}$. Method 3 is applicable up to $180^{\circ}$ angle of attack, although no test verification has been obtained for $\alpha>60^{\circ}$. DATCOM suggests that methods 1 and 2 should be used for small angles of attack whenever possible, as larger amounts of substantiated data is available, while method 3 should be used for higher angles of attack.

\section{Drag and Axial Coefficients:}

For body drag at angles of attack DATCOM presents 4 methods:

1. The method of Hopkins [12], which was discussed in Section 2.2.1.3.

2. The method of Allen and Perkins [11], which was discussed in Section 2.2.1.2. 
3. A method by H. Kelly [15], which is stated to be accurate to $\pm 10 \%$ for bodies of high fineness ratio, but is not accurate for low fineness ratio bodies. It assumes that the viscous contribution along the body is equal to the steady-state drag of a section of infinite cylinder placed normal to the flow with velocity $V \sin \alpha$. It is valid for blunt-based bodies at $\alpha<10^{\circ}$ and $(2 f \tan \alpha)<5$.

4. The method by Jorgensen [13] for calculating the axial-force coefficient, which was described in Section 2.2.1.4.

For each method the drag coefficient due to angle of attack is added to the body zero-lift drag coefficient.

DATCOM suggests that methods $1-3$ should be used for low angles of attack, while method 4 should be used for high angles of attack, although no substantiating test data is available for subsonic speeds for this method.

\section{Pitching-Moment Coefficients:}

For pitching-moment coefficients in the nonlinear angle of attack range DATCOM presents 2 methods:

1. The method by Hopkins [12], as was discussed in Section 2.2.1.3.

2. The method by Jorgensen [13], as was discussed previously in Section 2.2.1.4.

DATCOM states that method 1 is satisfactory up to angles of attack of about $12^{\circ}$, again due to the small angle approximation, and suggests that this method should be used up to this angle to attack. Method 2 is stated to be in fairly good agreement with experimental 
data up to about $20^{\circ}$ angle of attack, but significantly underestimates the data at higher angles of attack.

\subsubsection{D. Perrault, 2002}

In his doctoral thesis, Perrault [7] derives equations for lift, drag, and pitching moment coefficients based on the work of Munk [9], Allen and Perkins [10] [11], and Hopkins [12]. Perrault gives the following equations for these coefficients, although does not state explicitly how they are derived:

$$
\begin{aligned}
& C_{l}=\left(k_{2}-k_{1}\right) \frac{S_{x 0}}{A} \sin 2 \alpha \cos \frac{\alpha}{2}+\eta C_{P c} \frac{\left(A_{p}-A_{p 0}\right)}{A} \sin ^{2} \alpha \cos \alpha \\
& C_{P H}=C_{D F \cdot(\alpha=0)}+\left(k_{2}-k_{1}\right) \frac{S_{\mathrm{r} 0}}{A} \sin 2 \alpha \sin \frac{\alpha}{2}+\eta C_{D x} \frac{\left(A_{p}-A_{p 0}\right)}{A} \sin ^{3} \alpha \\
& C_{M}=\left(k_{2}-k_{1}\right)\left(\frac{\mathrm{V}_{B}-S_{x 0}\left(x_{0}-x_{m}\right)}{A X}\right) \sin 2 \alpha \cos \frac{\alpha}{2}+\eta C_{D C}\left[\frac{A_{p}}{A}\left(\frac{x_{m}-x_{p}}{X}\right)-\frac{\left.A_{p 0}\left(\frac{x_{m}-x_{p 0}}{A}\right)\right] \sin ^{2} \alpha}{X}\right.
\end{aligned}
$$

where $S_{x \theta}$ is the cross-sectional area of the hull at point $x_{\theta}, A_{p \theta}$ is the planform area from the nose to the point $x_{0}, x_{p 0}$ is the distance from the nose to the centroid of the planform area $A_{p}$, and $x_{0}$ is defined as it was in (20).

Perrault makes the statement that the normal force on the hull will be equal to the lift force. Ward [16] showed that the normal force acts at an angle that bisects the angle between the normal line of the flow and the normal line of the vehicle longitudinal axis. Perrault's statement is true for the potential term in the force equation, as Ward performed a potential flow analysis, but we must be careful not to include the viscous 
force term in this statement. The viscous term in the normal coefficient will not contain the term, $\cos \alpha$, as we saw previously in the work of Jorgensen [13]. Thus the normal force coefficient will not equal the lift force coefficient.

Due to the axisymmetric body that Perrault is analyzing, the center of pressure for the hull, which Perrault terms the center of effort, is common for pitch and sideslip. He does not include an equation for $C_{N}$. It is possible that he has assumed it to be equal to the lift coefficient, which becomes less accurate as the angle of attack increases.

The center effort is calculated as the following (similar to (23) presented by Jorgensen):

$$
x_{1:}=\frac{X C_{M}}{C_{N}}
$$

\subsubsection{Hull Forces Calculation}

Based on the works reviewed in Sections 2.2.1.1 to 2.2.1.7, it was decided that the most appropriate formulation for our AUV simulation is the method of Jorgensen [13] due to the ability to cover large angles of attack. If the vehicle is required to stop or hover in a cross-flow, angles of attack on the hull must be able to handle the full $360^{\circ}$ range. As DATCOM indicated, Jorgensen's equations are the only formulations appropriate for this range, although DATCOM also lists the following warnings:

- In the calculation of $C_{L}$, no test verification has been obtained for $\alpha>60^{\circ}$.

- In the calculation of $C_{D}$, no substantiating test data is available for subsonic speeds. 
- In the calculation of $\mathrm{C}_{\mathrm{M}}$, the results are in fairly good agreement with experimental data up to about $20^{\circ}$ angle of attack, but it significantly underestimates the data at higher angles of attack.

Jorgensen presents his formulation as a static formulation, meaning that it calculates hull forces and moments at a fixed angle of attack at steady state. As the vehicle will experience accelerations, we are also interested in a dynamic formulation. We present the static and dynamic formulations in Sections 2.2.2.1 and 2.2.2.2.

\subsubsection{Static Formulation}

Unlike Munk [9], Allen and Perkins [10, 11], and Hopkins [12], Jorgensen does not include the apparent mass factor in the potential term in the calculation of both the normal and pitching moment coefficients. Excluding this term has an insignificant effect for high fineness ratio bodies, as this term has a value of close to one. However this is not always the case with AUVs, so we will modify Jorgensen's equations to include this added mass term:

$$
\begin{array}{ll}
C_{N}=\left(k_{2}-k_{1}\right) \frac{S_{b}}{A} \sin 2 \alpha^{\prime} \cos \frac{\alpha^{\prime}}{2}+\eta C_{D c} \frac{A_{p}}{A} \sin ^{2} \alpha^{\prime} & 0^{\circ} \leq \alpha \leq 180^{\circ} \\
C_{A}=C_{D F\left(\alpha=0^{\circ}\right)} \cos ^{2} \alpha^{\prime} & 0^{\circ} \leq \alpha \leq 90^{\circ} \\
C_{A}=C_{D F\left(\alpha=180^{\circ}\right)} \cos ^{2} \alpha^{\prime} & 90^{\circ} \leq \alpha \leq 180^{\circ} \\
C_{M}=\left(k_{2}-k_{1}\right)\left[\frac{\left.V_{B}-\frac{S_{b}\left(l-x_{m}\right)}{A X}\right] \sin 2 \alpha^{\prime} \cos \frac{\alpha^{\prime}}{2}+\eta C_{D c} \frac{A_{p}}{A}\left(\frac{x_{m}-x_{p}}{X}\right) \sin ^{2} \alpha^{\prime}}{X}\right. & 0^{\circ} \leq \alpha \leq 90^{\circ}
\end{array}
$$


$C_{M}=-\left(k_{2}-k_{1}\right)\left[\frac{V_{B}-S_{h} x_{m}}{A X^{\prime}}\right] \sin 2 \alpha^{\prime} \cos \frac{\alpha^{\prime}}{2}+\eta C^{\prime} \frac{A_{p}}{A}\left(\frac{x_{m}-x_{p}}{X}\right) \sin ^{2} \alpha^{\prime}$

$90^{\circ} \leq \alpha \leq 180^{\circ}$

where

$$
\begin{array}{ll}
\alpha^{\prime}=\alpha & \text { for } 0^{\circ} \leq \alpha \leq 90^{\circ} \\
\alpha^{\prime}=180^{\circ}-\alpha & \text { for } 90^{\circ} \leq \alpha \leq 180^{\circ}
\end{array}
$$

Due to the fact that the equations shown in (28) are intended for a blunt based body, we will use the maximum cross-section of the hull for $S_{b}$.

The angle of attack on the hull, $\alpha$, is defined as the following:

$$
\alpha=\tan ^{-1}\left(\frac{\sqrt{v_{C E}^{2}+w_{C E}^{2}}}{u_{C E}}\right)
$$

Note that, based on (29), $\alpha$ will lie between $0^{\circ}$ and $180^{\circ}$. The $180^{\circ}$ to $360^{\circ}$ range is accounted for by the rotation angle, $\Phi$, specifying the velocity vector location in the body-fixed $Y-Z$ plane (see Figure 2.7). The subscript $C E$ designates the center of pressure of the hull, which we term the center of effort [7]. Due to the symmetrical characteristics of most AUVs, the center of effort is located on the longitudinal axis, at a longitudinal location that moves aft with increasing angle of attack [13]. It generally lies ahead of the center of mass and can even extend ahead of the nose at low angles of 
attack. The velocity components at the center of effort can be calculated from those at the center of mass as follows:

$$
\left[\begin{array}{c}
u_{C E} \\
v_{C E} \\
w_{C K}
\end{array}\right]=\left[\begin{array}{c}
u \\
v \\
w
\end{array}\right]+\left[\begin{array}{l}
p \\
q \\
r
\end{array}\right] \times\left[\begin{array}{c}
x_{C E} \\
y_{C E} \\
z_{C E}
\end{array}\right]
$$

where the longitudinal position of the center of effort, $x_{C E}$, is calculated by (27) and $y_{C E}$ and $z_{C E}$ are equal to the distance from the center of mass to the longitudinal axis of the hull along the $y$ and $z$ body-fixed axes.

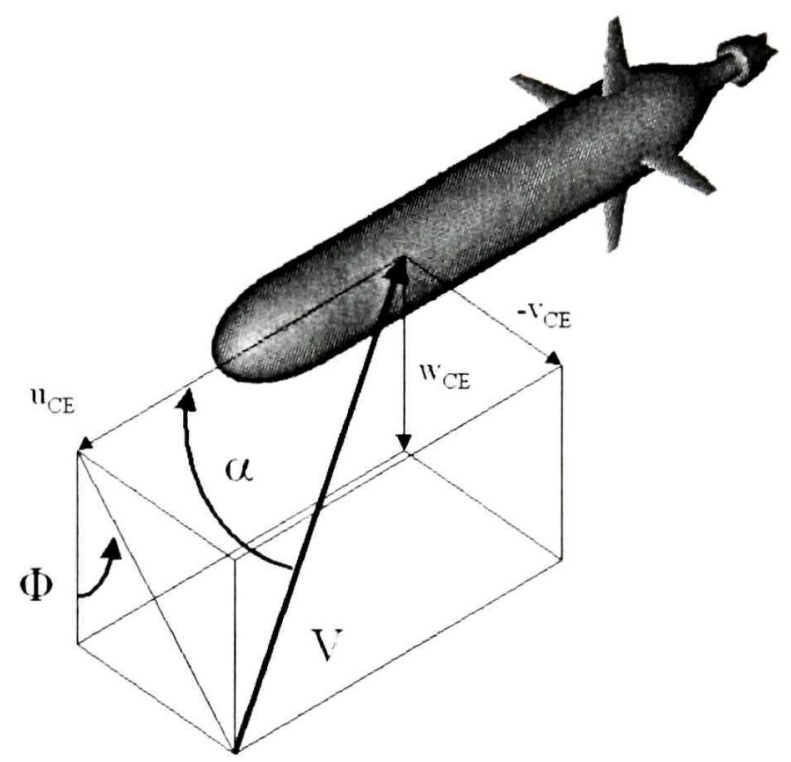

Figure 2.7 - Illustration of Hull Angles

The values for $C_{D c}$ in equation (28) are a function of Reynolds Number, which Jorgensen [13] presents for subcritical Mach Numbers, based on data from various researchers (see Figure 2.6). Jorgensen also presents values for the cross-flow drag proportionality factor, $\eta$ (see Figure 2.8 ), which is a funtion of the fineness ratio of the vehicle. 


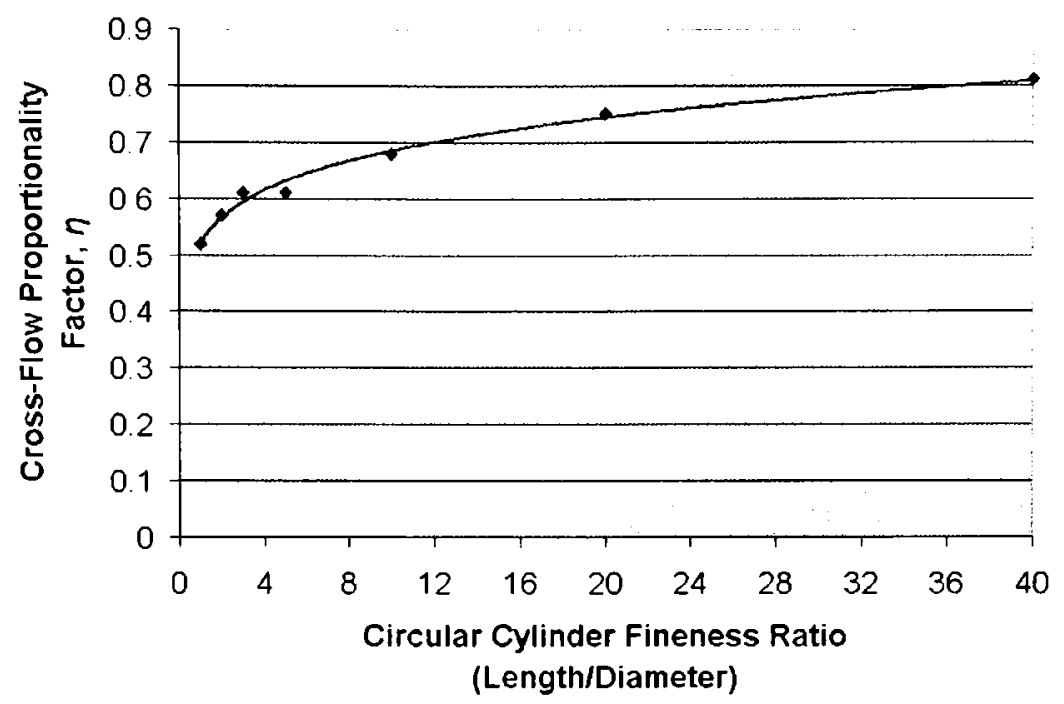

Figure 2.8-Cross Flow Drag Proportionality Factor

The forces on the hull expressed in the body-fixed frame are computed as follows:

$$
\left[\begin{array}{c}
X_{H u l l} \\
Y_{H u l l} \\
Z_{H u l l}
\end{array}\right]=\frac{1}{2} \rho A V_{(\because:}^{2}\left[\begin{array}{ccc}
-1 & 0 & 0 \\
0 & \sin \Phi & 0 \\
0 & -\cos \Phi & 1
\end{array}\right]\left[\begin{array}{c}
C_{f} \\
C_{N} \\
0
\end{array}\right]
$$

where $V_{C E}$ is the total velocity at the location of the center of effort and $\Phi$ is the rotation angle (see Figure 2.7), defined as:

$$
\Phi=\tan ^{-1}\left(\frac{-v_{C E}}{w_{C E}}\right)
$$

The point of application of the forces is at the center of effort of the hull. Therefore the moments about the center of mass due to the hull forces are calculated as the applied hull force multiplied by the moment arm from the center of mass to the center of effort:

$$
\mathbf{M}_{\text {Hull }}=\mathbf{r}_{\mathrm{CE}} \times \mathbf{F}_{\mathrm{Hull}}
$$


Note that based on the definition of the location of the center of effort, described in (27), the method of calculating moments in (33) will produce the same result as a moment calculated with the use of the moment coefficient, described in (28).

\subsubsection{Dynamic Formulation}

To extend the static formulations to a dynamic formulation, we will follow the method presented in Jones and DeLaurier [18] for the estimation of hull forces on airships. Jones and Delaurier use Allen and Perkins' [11] formulation to calculate hull forces, which are expressed in a body-fixed frame. The general concept of the dynamic model is to discretize the body into small segments (we will call them panels, see Figure 2.9) and calculate the hull forces and moments on each panel based on the local velocity of the center of the panel. Then we sum the forces and moments over all the panels to obtain the total force and moment on the hull. This process is performed during the simulation, and the number of calculations used to determine the total force on the hull is dependent on the number of discretized panels. We follow the procedure of Jones and DeLaurier [18], but with the formulation described in (28) instead of Allen and Perkins' formulation. The derivation of the dynamics equations is included in Appendix A.

The dynamic formulation varies from the static formulation by the following points:

- An inertial term is introduced due to accelerations and rotations of the fluid around the vehicle 
- The forces and moments on the vehicle are determined based on the local velocity of each panel, which will vary along the length of the hull if the vehicle is undergoing rotation

- As the Reynolds Number can change along the length of the hull, the local cross-flow drag coefficient can vary for each panel (see Figure 2.6)

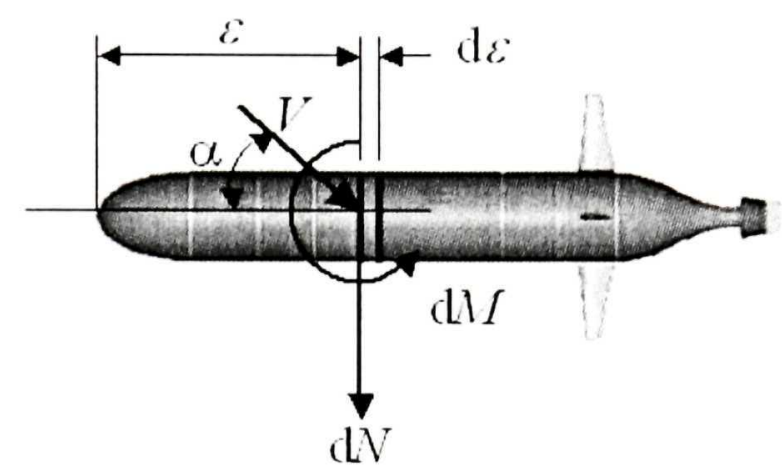

Figure 2.9 - Individual Panel on Discritized Hull

\subsection{Control Plane Forces}

Hydrodynamic control planes provide orientation control for the vehicle while it is in motion. Traditionally the forces are decomposed into lift and drag forces, perpendicular and parallel to the incoming fluid flow, respectively (see Figure 2.10).

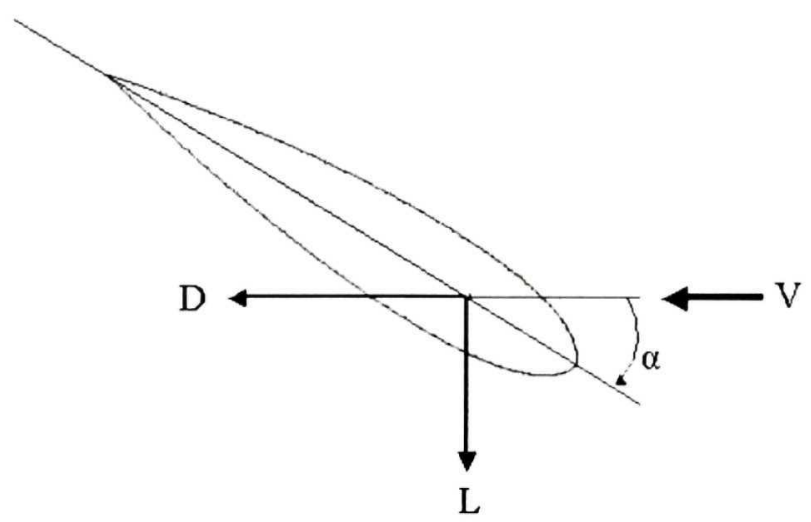

Figure 2.10 - Illustration of Lift and Drag on an Airfoil 
Lift and Drag coefficients for 2-D sections are readily available for a wide variety of wing sections at angles of attack up to the point of stall (e.g. [19]). However, we desire a simulation that is capable of the full $360^{\circ}$ range of angles. Few researchers have looked at such a wide range of angles, due to the limited usefulness of angles of attack beyond stall in aircraft operations. Two researchers that have looked at airfoil behavior at large angles of attack are Riegels [20] and Critzos et. al. [21] (see Figures 2.11 and 2.12). In our simulation of C-SCOUT, we will use the full $360^{\circ}$ range of lift and drag coefficients, which are each a function of angle of attack, as provided by Critzos et. al. [21].

Lift Coefficient Data

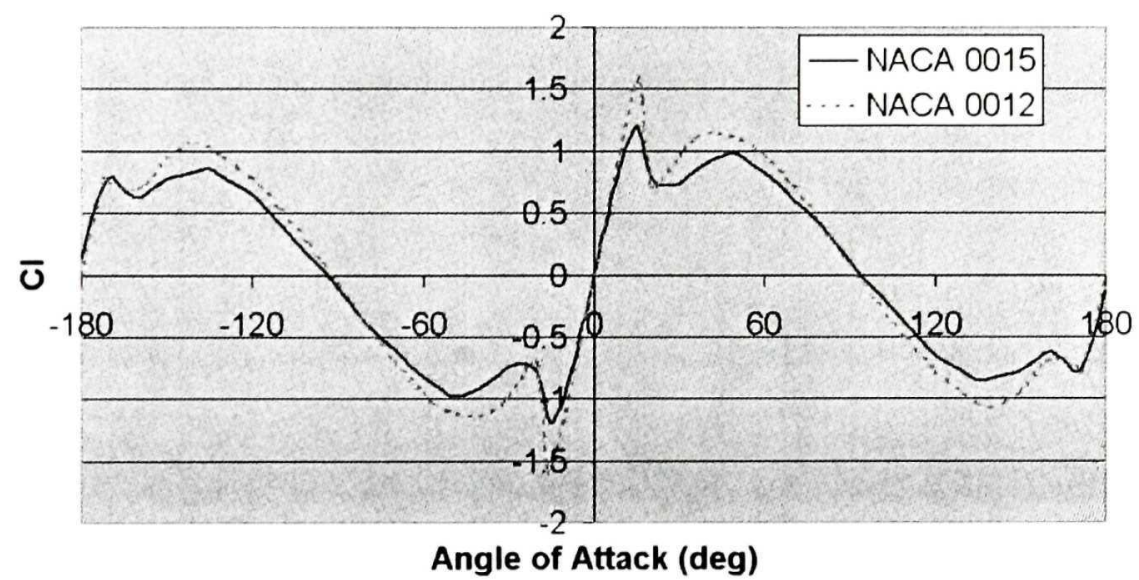

Figure 2.11 - Lift Coefficient Data Over $360^{\circ}$ Range for NACA 0012 [20] and NACA 0015 [21] Wing Sections 


\section{Drag Coefficient data}

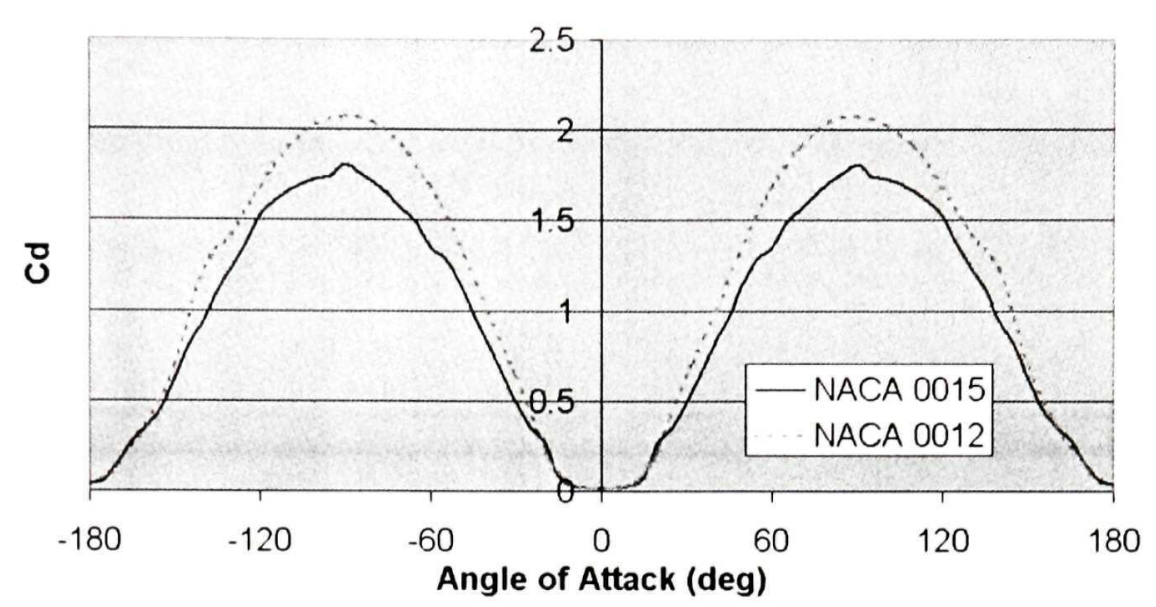

Figure 2.12 - Drag Coefficient Data Over $360^{\circ}$ Range for NACA 0012 [20] and NACA 0015 [21] Wing Sections

Modifying the 2-D wing section lift and drag coefficients to apply to 3-D wing can be accomplished with methods such as those presented by McCormick [22]:

$$
\begin{gathered}
C_{L}=\frac{a_{e} C_{1}}{a_{e}+2\left(\frac{a_{e}+4}{a_{e}+2}\right)} \\
C_{D}=f_{C D} C_{d}
\end{gathered}
$$

where $a_{e}$ is the effective aspect ratio of the plane, defined below, $C_{L}$ and $C_{D}$ are the 2-D section lift and drag coefficients, respectively, and $f_{C D}$ is a scale factor from McCormick [22], Figure 4.11. Note that (51) is intended for the linear angle of attack range before stall, but is assumed valid here for the nonlinear range as well. The effective aspect ratio of the plane is defined as:

$$
a_{e}=\frac{b_{C P}^{2}}{A_{C P}}
$$

where $b_{C P}$ is the span (twice the distance from the root to the tip of the control plane) and $A_{C P}$ is the planform area of a set of control planes, without the area included inside 
the hull. We do not include the area inside the hull (inclusion of this area is commonly performed in aircraft analysis) due to the large hull size compared to the control plane size. Including this area inside the hull would increase the control plane area by almost 2-fold.

The lift and drag coefficients for the horizontal planes are a function of the angle of attack and the plane deflection. The lift and drag coefficients for the vertical planes are a function of the angle of sideslip and the plane deflection. The angle of attack and sideslip angle for the horizontal control planes are computed as:

$$
\begin{array}{r}
\left(\alpha_{C P}\right)_{h o r}=\tan ^{-1}\left(\frac{w_{C P}}{u_{C P}}\right) \\
\left(\beta_{(P)}\right)_{h o r}=\tan ^{-1}\left(\frac{v_{(P P}}{\sqrt{u_{C P}^{2}+w_{C P}^{2}}}\right)
\end{array}
$$

while for the vertical control planes, they are computed as:

$$
\begin{gathered}
\left(\alpha_{C P}\right)_{v e r}=\tan ^{-1}\left(\frac{w_{C P}}{\sqrt{u_{C P}^{2}+v_{C P}^{2}}}\right) \\
\left(\beta_{C P}\right)_{v e r}=\tan ^{-1}\left(\frac{v_{C P}}{u_{C P}}\right)
\end{gathered}
$$

Estimation of lift and drag forces on the planes in 3-D fluid flow is another issue to be considered. Lift and drag coefficients are typically provided as a function of angle of attack only. Thus we must make an assumption when the plane experiences sideslip. To account for this, we calculate the control plane's lift and drag forces, neglecting the 
cross-plane velocity component. Lift and drag on the horizontal control planes are calculated as follows:

$$
\begin{aligned}
& \left(L_{(P)}\right)_{h u r}=\frac{1}{2} \rho A C_{L}\left[V_{C,} \cos \left(\beta_{(p)}\right)_{h u r}\right]^{2} \\
& \left(D_{(p)}\right)_{h o r}=\frac{1}{2} \rho A C_{D}\left[V_{(P,} \cos \left(\beta_{C P}\right)_{h o r}\right]^{2}
\end{aligned}
$$

while on the vertical control planes, they are calculated as follows:

$$
\begin{aligned}
& \left(L_{C P}\right)_{v e r}=\frac{1}{2} \rho A C_{L}\left[V_{C P} \cos \left(\alpha_{C \cdot P}\right)_{v e r}\right]^{2} \\
& \left(D_{C P}\right)_{v e r}=\frac{1}{2} \rho A C_{D}\left[V_{C P} \cos \left(\alpha_{C P}\right)_{v e r}\right]^{2}
\end{aligned}
$$

The control plane forces in the body frame, acting at the center of pressure of the control planes (defined below), are computed as:

$$
\begin{aligned}
& {\left[\begin{array}{c}
X_{C P} \\
Y_{C P} \\
Z_{C P}
\end{array}\right]_{h o r}=\left[\begin{array}{ccc}
-\cos \left(\alpha_{C P}\right)_{h o r} & 0 & \sin \left(\alpha_{C P}\right)_{h o r} \\
0 & 1 & 0 \\
-\sin \left(\alpha_{C P}\right)_{h o r} & 0 & -\cos \left(\alpha_{C P}\right)_{h o r}
\end{array}\right]\left[\begin{array}{c}
D_{C P} \\
0 \\
L_{C P}
\end{array}\right]_{h o r}} \\
& {\left[\begin{array}{c}
X_{C P} \\
Y_{C P} \\
Z_{C P}
\end{array}\right]_{v e r}=\left[\begin{array}{ccc}
-\cos \left(\beta_{C P}\right)_{v e r} & \sin \left(\beta_{C P}\right)_{v e r} & 0 \\
-\sin \left(\beta_{C P}\right)_{v e r} & -\cos \left(\beta_{C P}\right)_{v e r} & 0 \\
0 & 0 & 1
\end{array}\right]\left[\begin{array}{c}
D_{C P} \\
L_{C P} \\
0
\end{array}\right]_{v e r}}
\end{aligned}
$$

External moments on the vehicle due to the forces on the control planes are expressed in the body frame as the following:

$$
\mathbf{M}_{\mathrm{CP}}=\mathbf{r}_{\mathrm{CP}} \times \mathbf{F}_{\mathrm{CP}}
$$


where $\mathbf{r}_{\mathrm{CP}}$ is a vector from the center of mass of the vehicle to the center of pressure of the control plane. The center of pressure of the control plane is assumed to be at the quarter-chord point of the section at $42 \%$ of the halfspan out from the root chord [7].

\section{$2.4 \quad$ Rear Thruster}

The rear thruster is simply modeled as a force exerted along the longitudinal axis of the vehicle. At equilibrium, this thrust will equal the drag of the vehicle, and no acceleration will occur. Once the vehicle is in motion, the thrust is inversely proportional to the forward velocity of the vehicle, which simulates a propulsor with constant power output:

$$
T=\frac{T_{0} V_{0}}{u}
$$

where $T$ is the thrust and $T_{0}$ is equal to the drag of the vehicle at its initial equilibrium velocity, $V_{0}$.

\subsection{Model Validation Using ARCS Vehicle}

One of the valuable aspects of an AUV simulation is its ability to evaluate a vehicle's performance before it has been constructed. We wish to know how the performance of the Baseline Configuration will compare to that of the Fully-Actuated Configuration. Our vehicle simulation is coded in Simulink ${ }^{\circledR}$. Before using it to evaluate C-SCOUT's performance, we wish to know if it is accurate. However, validation data is not currently available for C-SCOUT, though it is expected to become available for the Baseline 
Configuration in the near future. For validation, we will model the ARCS vehicle, for which extensive experimental data is available.

\subsubsection{The ISE ARCS Vehicle}

ARCS [31] is an AUV developed by International Submarine Engineering (ISE) and used as a testbed for evaluating AUV technologies (see Figure 2.13). ARCS consists of a hull, six independently controllable planes (two horizontal foreplanes, two horizontal aftplanes, and two vertical rudder planes) and two nose bulges. The hull has a hemispherical nose section, a cylindrical midsection and a conical tail section. The geometry of the vehicle was taken from scale drawings, while the manufacturer provided the location of the center of mass and center of buoyancy. The vehicle was assumed to be neutrally buoyant and the control planes are assumed to each be a NACA 0018 profile.

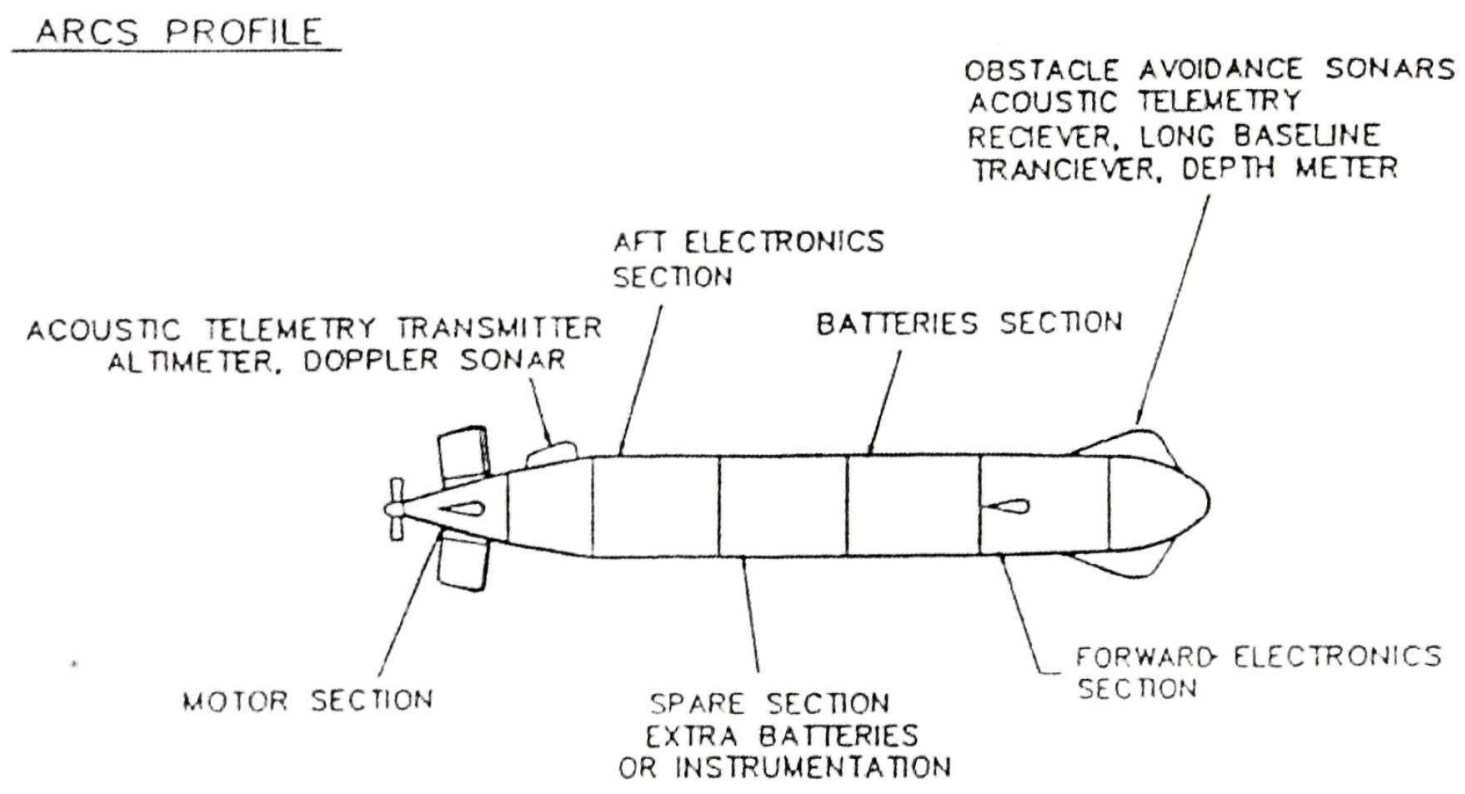

Figure 2.13 - Side View of the ISE ARCS Vehicle 
Unfortunately, data for the lift and drag coefficients for the full $360^{\circ}$ range could not be found for the NACA 0018 profile. Therefore we will follow a slightly different method of calculating the lift coefficient than that shown in (51). We will use the lift coefficient data for the NACA 0015 profile [21] (see Figure 2.11) multiplied by a scale factor. This scale factor will include the effects of the change from an infinite aspect ratio profile to a finite aspect ratio wing. interference effects, and the effects of a physically different profile. Hopkin and Hertog [23] provide lift curve slopes in the linear range for each of the control planes on ARCS based on experimental data. These lift curve slopes are listed as 3.2, 1.8, and 2.0 per rad for the foreplanes, aftplanes, and rudders respectively. For the NACA 0015 profile lift coefficient data, shown in Figure 2.11, the slope in the linear range is 5.45 per rad. Therefore in order to adapt the lift coefficient data of the NACA 0015 profile to the ARCS control planes, we will use scale factors of 0.588 , 0.331 , and 0.367 for the foreplanes, aftplanes, and rudders respectively (i.e. for the foreplanes the lift curve slope scale factor is $3.2 / 5.45=0.588$ ). We make the assumption that this factor will remain constant over the $360^{\circ}$ range, and that the angle of attack at which stall occurs will not be altered.

Hopkin and Hertog [23] do not provide drag coefficients for the control planes, as the drag is typically calculated as a function of the lift coefficient for the linear angle of attack range. Therefore in order to model the full $360^{\circ}$ angle of attack range, we will use a scale factor based on the profile data we have available. We will make the assumption that the difference in the drag coefficient between the NACA 0018 profile and the NACA 0015 profile is the same as the difference between the NACA 0015 profile and 
the NACA 0012 profile (see Figure 2.12). At $90^{\circ}$ plane deflection, the drag coefficient for the NACA 0015 profile is $87 \%$ of the drag coefficient for the NACA 0012 profile. Therefore we will apply a scale factor of 0.87 to the NACA 0015 profile drag coefficient data to estimate the NACA 0018 profile drag data. We then modify this 2-D drag coefficient data to a 3-D control plane using (52), which generates a scale factor of $f_{C D}=$ 1.5. Thus a total scale factor of $(0.87)(1.5)=1.31$ is applied to the NACA 0015 profile drag coefficient data to obtain the NACA 0018 3-D drag coefficient data over the $360^{\circ}$ angle of attack range.

The nose bulges can exert a substantial moment on the vehicle in the presence of sideslip. We can account for these bulges by including them as immovable planes of extremely low aspect ratio (i.e. $<1$ ). We will consider the nose bulges to have a lift curve slope of 1.2 per rad [6]. Adapting this to the NACA 0015 lift coefficient data, similarly to that described in the previous paragraph, we use a scale factor of 0.221 . For the drag coefficient, we will assume the drag is the same as for the control planes and use a scale factor of 0.87 to adapt the NACA 0015 profile drag coefficient data. Using (52), a scale factor of $f_{C D}=0.214$ is obtained. Thus a total scale factor of $(0.87)(0.214)=$ 0.186 is applied to the NACA 0015 profile drag coefficient data to obtain the 3-D drag coefficient data over the $360^{\circ}$ angle of attack range.

\subsubsection{Model Validation}

We have two formulations for the estimation of the hull forces: a static formulation and a dynamic formulation, each based on Jorgensen`s model. In order to choose which of 
these formulations to use in our model, we require a validation based on experimental data. Using physical parameters of the ARCS vehicle in our simulation, we can compare the results to field test data performed by Hopkin and den Hertog [23]. Hopkin and den Hertog list turning diameters for three vehicle velocity and rudder deflection combinations of the ARCS. Although this is not a thorough validation, it does give us an indication of the accuracy of our simulation. Table 2.2 compares the results of our simulation and the field test data:

\begin{tabular}{|l|c|c|c|}
\hline \multicolumn{4}{|c|}{ Turning Diameters } \\
\hline & $\begin{array}{l}25^{\circ} \text { rudder deflection } \\
\text { at } 1.5 \mathrm{~m} / \mathrm{s}\end{array}$ & $\begin{array}{l}25^{\circ} \text { rudder deflection } \\
\text { at } 2.5 \mathrm{~m} / \mathrm{s}\end{array}$ & $\begin{array}{l}15^{\circ} \text { rudder deflection } \\
\text { at } 2.5 \mathrm{~m} / \mathrm{s}\end{array}$ \\
\hline Measured Field Data & $32.9 \mathrm{~m}$ & $34.8 \mathrm{~m}$ & $51.8 \mathrm{~m}$ \\
\hline Static Formulation & $32.5 \mathrm{~m}$ & $33.1 \mathrm{~m}$ & $58.8 \mathrm{~m}$ \\
\hline Dynamic Formulation & $40.2 \mathrm{~m}$ & $37.4 \mathrm{~m}$ & $60.2 \mathrm{~m}$ \\
\hline
\end{tabular}

Table 2.2 - Measured and Predicted Turning Diameters for the ARCS Vehicle

Surprisingly, the simulated turning diameters using the static formulation appear to more closely match the measured field data than the dynamic formulation. We would expect that the dynamic formulation would more accurately represent the vehicle motions, as the vehicle is undergoing acceleration. Discrepancies between the results could be due to assumptions made in modeling the control planes and bulges of the ARCS vehicle. Due to a lack of validation data for C-SCOUT, and since the static formulation appears more accurate for ARCS, we will use the static formulation to model C-SCOUT. 


\section{Chapter 3 Adaptation Of The Vehicle Model to}

\section{C-SCOUT}

One of the valuable aspects of an AUV simulation is its ability to evaluate a vehicle's performance before it has been constructed. Thus it can be utilized as a predictive tool for the AUV designer. The C-SCOUT vehicle, under development at Memorial University of Newfoundland (MUN), has been built in its most basic configuration: the Baseline Configuration. We wish to know how the performance of the Baseline Configuration will compare to another possible configuration: the Fully-Actuated Configuration. With our AUV dynamics simulation, we can compare the predicted performance of the two vehicle configurations and possibly determine situations where one vehicle configuration will be advantageous over the other.

C-SCOUT is a modular AUV, and can be arranged in multiple configurations. In this thesis we are concerned with two configurations: the Baseline Configuration and the Fully Actuated Configuration (see Figure 1.1). Each configuration adds modules to a basic hull, thereby lengthening the vehicle. The basic hull has an ellipsoidal nose, a 
cylindrical mid-section, and a cubic spline tail section. Contained in the basic hull are the components required for AUV operations, such as obstacle avoidance sonar, a computer system, a navigation and orientation system, an energy storage system, payload such as cameras or oceanographic sensors, and ballast. The vehicle always maintains neutral buoyancy and therefore relies on hydrodynamic forces generated by the control planes or through-body thrusters for depth control. Except for the pressure vessel, which contains the electronics of the vehicle, the hull is free-flooding, making sealing unnecessary. Conversion from one configuration to another is nominally a simple matter of removing bolts and inserting or removing a module. The rear thruster is common to both configurations.

Drag resistance testing has been performed on the basic hull in the tow tank at Memorial University for forward velocities up to $2.5 \mathrm{~m} / \mathrm{s}$ [24]. From the experimental results we can estimate the drag coefficient on the basic hull without the control planes. A drag coefficient of $C_{D F\left(\alpha=0^{\circ}\right)}=0.0077$, based on the wetted area of the hull, achieves a good fit to the empirical data (see Figure 3.1). We will assume that the drag coefficient stays constant for the Baseline and Fully Actuated Configurations. However, due to the larger surface area of the Fully Actuated Configuration, its drag force will be higher. 


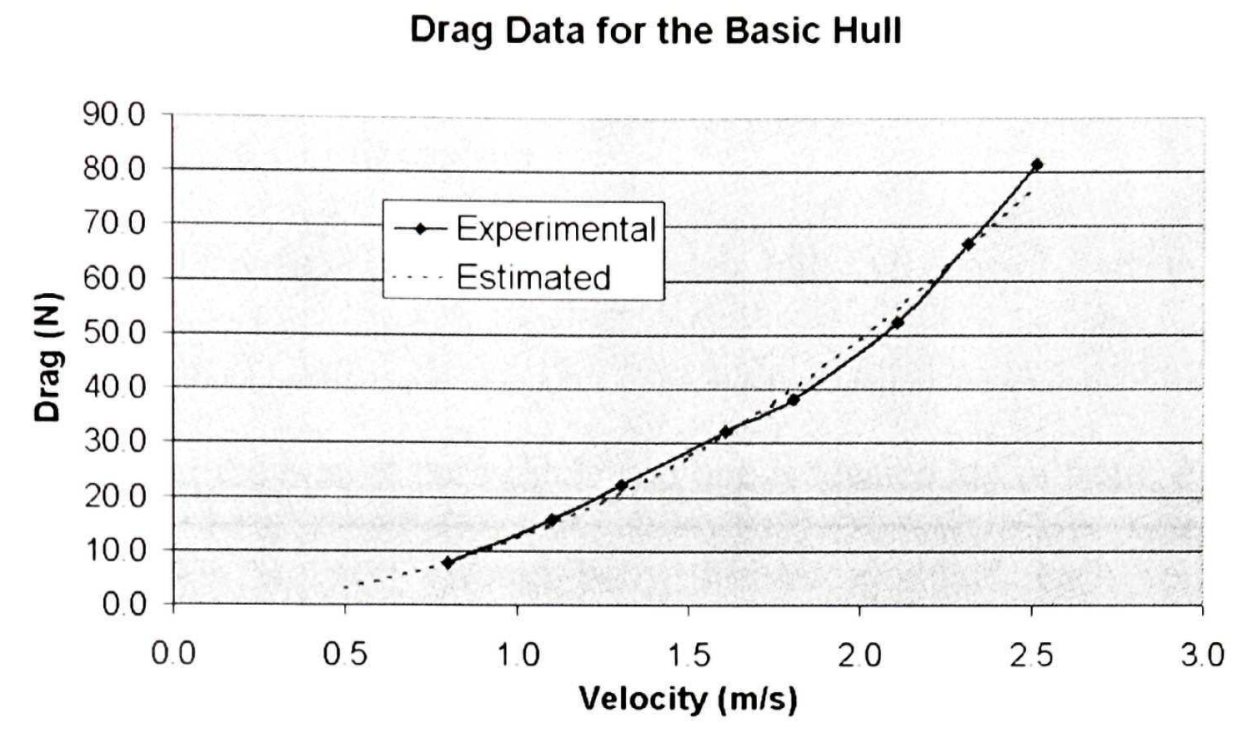

Figure 3.1 - C-SCOUT Basic Hull Drag Data

\subsection{Baseline Configuration}

The Baseline Configuration adds a control plane module to the basic hull at the rear of the vehicle (see Figure 3.2). The control plane module consists of two vertical control planes, two horizontal control planes, and an independently controller actuator for each plane.

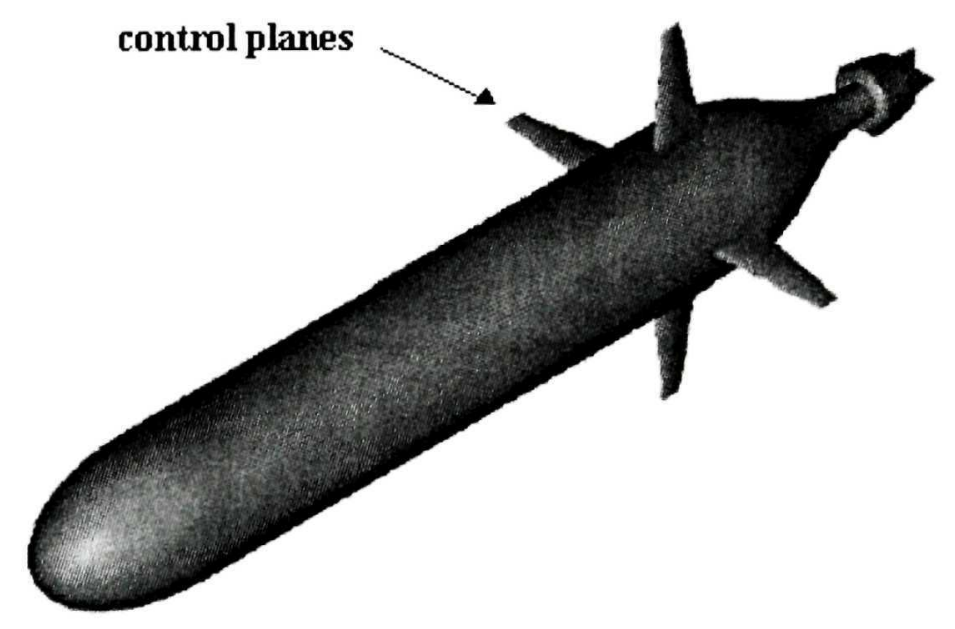

Figure 3.2-C-SCOUT Baseline Configuration 


\subsection{Fully Actuated Configuration}

The Fully Actuated Configuration adds to the basic hull two control plane modules, each containing two vertical control planes, two horizontal control planes, and actuators. One module is located at the rear of the vehicle and one is located at the front. Also included in this configuration are two through-body thruster modules, each containing two horizontal thrusters and one vertical thruster. One of these modules is located in front of the rear control plane module, and the other behind the front control plane module (see Figure 3.3).

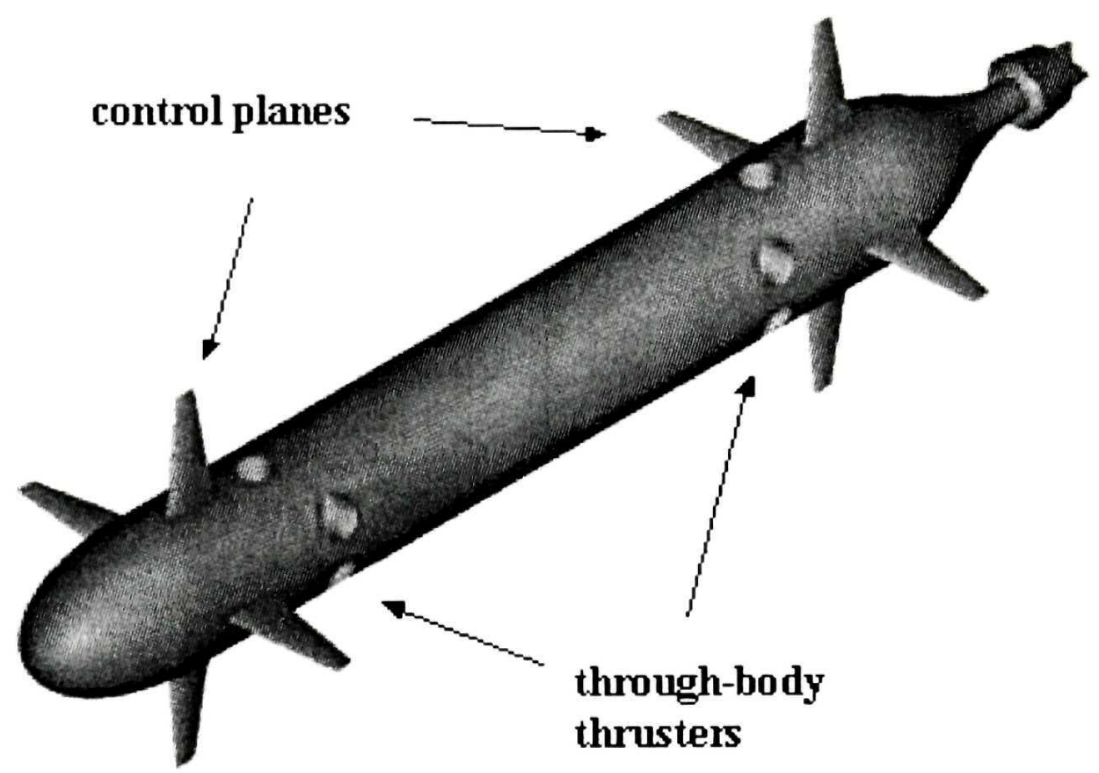

Figure 3.3-C-SCOUT Fully Actuated Configuration

The through-body thrusters are intended to improve the maneuverability of the vehicle at low speeds and to give the vehicle the ability to hover in a cross-flow. 


\subsubsection{Through-Body Thruster Implementation}

C-SCOUT uses through-body thrusters in its Fully Actuated Configuration, and we therefore require a method of modeling these thrusters in the vehicle simulation. Saunders [8] studied the effects of forward speed and vehicle orientation on throughbody thruster performance. He created a dynamics model of a through-body thruster accounting for the effects of yaw angle and forward velocity, specific to C-SCOUT. Here, we briefly discuss that model and how it is incorporated into our simulation.

Saunders uses blade element theory to approximate the hydrodynamic forces acting on the propeller. A schematic of the relevant forces and velocities on the propeller is shown in Figure 3.4.

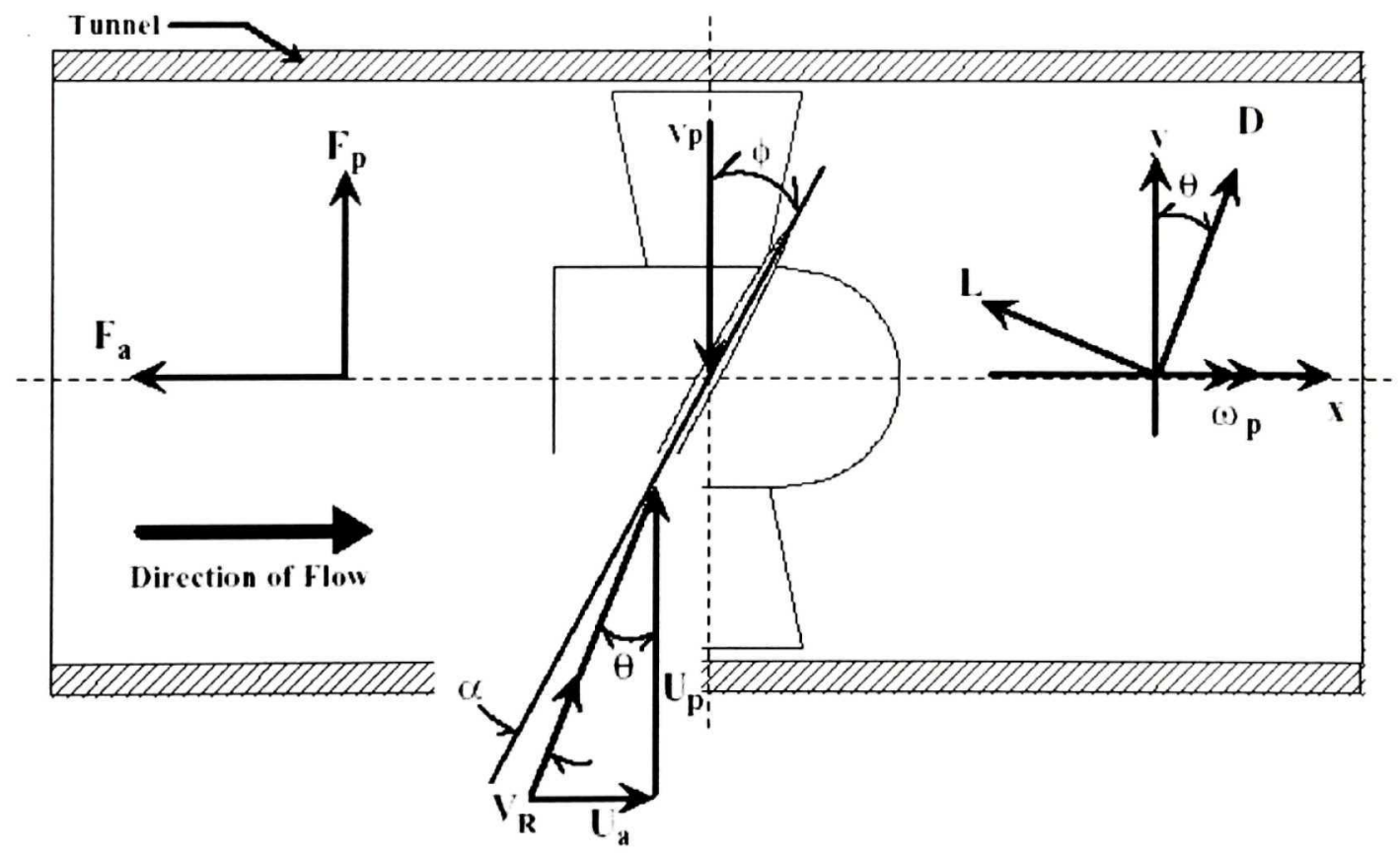

Figure 3.4 - Tunnel Configuration 
We can determine the tangential velocity of the propeller, $U_{p}$, based on the propeller speed according to the following:

$$
U_{p}=0.7 R_{p} \omega_{p}
$$

where $R_{D}$ is the radius of the propeller and $\omega_{p}$ is the speed of the propeller. The hydrodynamic pitch angle on the propeller, $\theta_{p}$, can then be calculated with:

$$
\theta_{p}=\tan ^{-1}\left(\frac{U_{a}}{U_{p}}\right)
$$

where $U_{a}$ is the axial water column velocity.

The angle of attack on the blade, $\alpha_{p}$, can be calculated as the pitch angle of the blade, $\phi_{p}$, minus the hydrodynamic pitch angle:

$$
\alpha_{p}=\phi_{p}-\theta_{p}
$$

The lift and drag on the propeller are calculated as:

$$
\begin{aligned}
& L_{p}=\left(\frac{1}{2} \rho V_{R}^{2} A_{D}\right) C_{L} \\
& D_{p}=\left(\frac{1}{2} \rho V_{R}^{2} A_{D}\right) C_{D}
\end{aligned}
$$

where $A_{D}$ is the tunnel duct area, $V_{R}$ is the resultant velocity on the propeller (described below), and the lift and drag coefficients are assumed to be simple sinusoids:

$$
\begin{gathered}
C_{L}=C_{L \max } \sin \left(2 \alpha_{p}\right) \\
C_{D}=C_{D \max }\left(1-\cos \left(2 \alpha_{p}\right)\right)
\end{gathered}
$$




$$
V_{R}=\sqrt{U_{a}^{2}+U_{p}^{2}}
$$

The hydrodynamic force, $F_{a}$, and hydrodynamic resistance, $F_{p}$, can be calculated with the following:

$$
\left[\begin{array}{c}
F_{a} \\
F_{p}
\end{array}\right]=\left[\begin{array}{cc}
\cos \theta_{p} & -\sin \theta_{p} \\
\sin \theta_{p} & \cos \theta_{p}
\end{array}\right]\left[\begin{array}{c}
L_{p} \\
D_{p}
\end{array}\right]
$$

The hydrodynamic torque on the propeller, $T_{p}$, although not used in our simulation of CSCOUT, is calculated as:

$$
T_{p}=0.7 R_{p} F_{p}
$$

In order to determine the axial water column velocity, $U_{a}$, Saunders [8] uses momentum theory for propellers operating in an incompressible fluid. The results of his analysis show that the tunnel thruster can be described by a first-order differential equation:

$$
F_{a}=\rho A_{D} l_{D} \gamma \dot{U}_{a}+a+b \omega_{p}\left|\omega_{p}\right|
$$

where $F_{a}$ is the output thrust, $A_{D}$ and $l_{D}$ are the duct area and length, respectively, $\gamma$ is an empirically determined added mass coefficient, $\dot{U}_{a}$ is the time derivative of the fluid velocity at the propeller, and $a$ and $b$ are vehicle-specific, experimentally determined coefficients, that vary with yaw angle and forward velocity of the vehicle. The first term in (76) represents the transient effects of the thruster, and the second and third terms represent the steady-state effects. (76) can be rearranged to solve for $\dot{U}_{a}$, which can then be integrated to obtain $U_{a}$ : 


$$
\dot{U}_{a}=\frac{F_{a}-a-b\left|\omega_{p}\right| \omega_{p}}{\rho A_{l,} l_{b j} \gamma}
$$

This relationship is illustrated in the flow diagram shown in Figure 3.5, where $K 1$ is equal to $\rho A_{D} l_{D} \gamma, \omega_{m}$ is the speed of the motor, $N$ is a ratio to transform motor RPM into propeller rad/sec, and the block entitled + Quad Map of Lift/Drag represents the process described in equations (66) to (75).

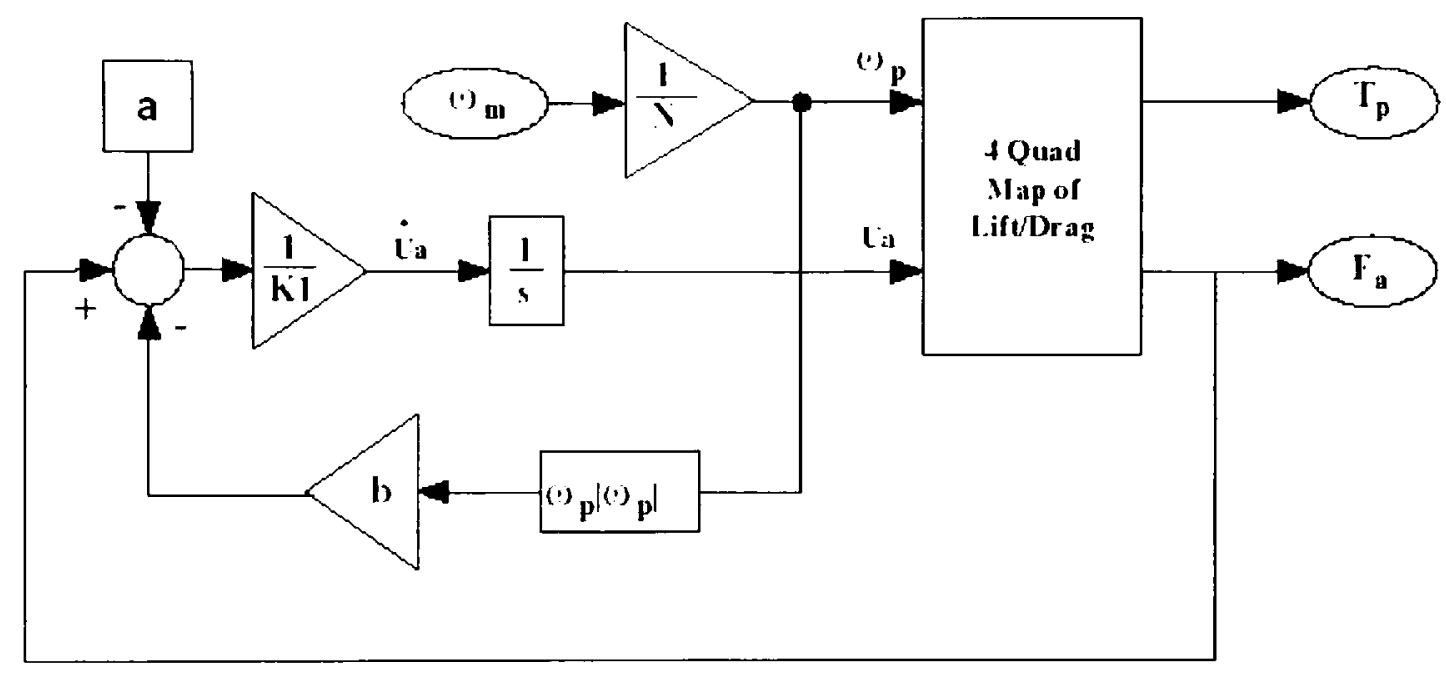

Figure 3.5 - Simulink Through-Body Thruster Model [8]

Saunders [8] obtained values for $a$ and $b$ for a forward through-body thruster mounted horizontally on C-SCOUT for sideslip angles from $-90^{\circ}$ to $90^{\circ}$ and forward vehicle velocities from 0 to $4 \mathrm{~m} / \mathrm{s}$. In our simulation of C-SCOUT, we assume that those values for $a$ and $b$ are independent of thruster position and orientation on the vehicle. During the simulation, $a$ and $b$ are selected from look-up tables based on the sideslip angle and forward speed. Velocity components at the center of the through-body thruster are computed as: 


$$
\left[\begin{array}{l}
u_{T T} \\
v_{T T} \\
w_{T T}
\end{array}\right]=\left[\begin{array}{c}
u \\
v \\
w
\end{array}\right]+\left[\begin{array}{c}
p \\
q \\
r
\end{array}\right] \times\left[\begin{array}{c}
x_{T T} \\
y_{T T} \\
z_{T T}
\end{array}\right]
$$

where $x_{T T}, y_{T T}$, and $z_{T T}$ are the locations of the center of the through-body thruster with respect to the center of mass in the body-fixed frame. In the calculation of the total forward velocity to be used in the look-up tables of $a$ and $b$, we obtain the projection of the velocity vector onto the plane defined by the axis of the though-body thruster and the longitudinal axis of the vehicle:

$$
\begin{aligned}
& \left(V_{T T}\right)_{h r r}=\sqrt{u_{T T}{ }^{2}+v_{T T}{ }^{2}} \\
& \left(V_{T T}\right)_{v e r}=\sqrt{u_{T T}{ }^{2}+w_{T T}{ }^{2}}
\end{aligned}
$$

where the subscripts, hor and ver describe a horizontal or vertical thruster orientation on the vehicle, respectively. The sideslip angle at the through-body thrusters is then computed as follows:

$$
\begin{aligned}
& \left(\beta_{T T}\right)_{h o r}=\tan ^{-1}\left(\frac{v_{T T}}{u_{T T}}\right) \\
& \left(\beta_{T T}\right)_{v e r}=\tan ^{-1}\left(\frac{w_{T T}}{u_{T T}}\right)
\end{aligned}
$$

The force exerted by each through-body thruster on the hull, $\mathbf{F}_{\mathrm{TT}}$, acting along the axis of the thruster, is calculated using (76). $\mathbf{F}_{\mathrm{TT}}$ will have only one non-zero component if the thruster is oriented parallel to one of the vehicle axes. The corresponding moment is then: 


$$
\mathbf{M}_{\mathrm{TT}}=\mathbf{r}_{\mathrm{TT}} \times \mathbf{F}_{\mathrm{TT}}
$$

where $\mathbf{r}_{\mathrm{TT}}=\left[\begin{array}{lll}x_{I T} & y_{I I} & z_{I T}\end{array}\right]^{\mathrm{T}}$.

\subsection{Vehicle Configuration Performance Comparisons}

We can now compare the performance of C-SCOUT's Baseline and Fully Actuated Configurations by examining the stability and turning diameter for each configuration at various vehicle speeds and control inputs. For the Baseline Configuration, the control inputs are control plane deflections. By contrast, the Fully Actuated Configuration can make use of control plane deflections or the through-body thrusters to control the vehicle.

\subsubsection{Stability}

Stability is a major concern for an AUV. We characterize an unstable vehicle as one that tends to follow an increasingly tightening spiral, from which it cannot recover. This can occur if the destabilizing moment generated by the hull normal force exceeds the stabilizing moment generated by the control planes.

In our simulation, we test for stability by setting an initial velocity and sideslip angle, with zero control plane deflections. Due to the sideslip angle, hydrodynamic forces are generated and the vehicle will begin to turn. A stable vehicle will return to a straight path, reducing the sideslip angle to zero. For an unstable vehicle, the sideslip angle will increase and the vehicle will enter a very tight spiral. 
The sideslip angle at which this instability occurs for the C-SCOUT Baseline Configuration is shown in Table 3.1. We observe that the vehicle instability increases at higher velocities. Note that this is with zero control plane deflection.

\begin{tabular}{|c|c|}
\hline $\begin{array}{c}\text { Velocity } \\
(\mathrm{m} / \mathrm{s})\end{array}$ & $\begin{array}{c}\text { Sideslip Angle Where } \\
\text { Instability Occurs }\end{array}$ \\
\hline 0.5 & $31^{\circ}$ \\
\hline 1.0 & $31^{\circ}$ \\
\hline 1.5 & $31^{\circ}$ \\
\hline 2.0 & $30^{\circ}$ \\
\hline 2.5 & $29^{\circ}$ \\
\hline 3.0 & $28^{\circ}$ \\
\hline 3.5 & $27^{\circ}$ \\
\hline 4.0 & $26^{\circ}$ \\
\hline
\end{tabular}

Table 3.1 - Sideslip Angle Where Instability Occurs for the Baseline Configuration

With the Fully Actuated Configuration, the forward control planes reduce the stability of the vehicle, as they lie forward of the center of mass (see Table 3.2). The stability does not appear to vary greatly with increasing velocity.

\begin{tabular}{|c|c|}
\hline $\begin{array}{c}\text { Velocity } \\
(\mathrm{m} / \mathrm{s})\end{array}$ & $\begin{array}{c}\text { Sideslip Angle Where } \\
\text { Instability Occurs }\end{array}$ \\
\hline 0.5 & $22^{\circ}$ \\
\hline 1.0 & $23^{\circ}$ \\
\hline 1.5 & $23^{\circ}$ \\
\hline 2.0 & $23^{\circ}$ \\
\hline 2.5 & $22^{\circ}$ \\
\hline 3.0 & $22^{\circ}$ \\
\hline 3.5 & $22^{\circ}$ \\
\hline 4.0 & $21^{\circ}$ \\
\hline
\end{tabular}

Table 3.2 - Sideslip Angle where Instability Occurs for the Fully Actuated Configuration 


\subsubsection{Turning Diameters}

In order to determine the turning diameter that each configuration is capable of achieving, based on control planes deflections, we design a test matrix for velocities from $0.5 \mathrm{~m} / \mathrm{s}$ to $4.0 \mathrm{~m} / \mathrm{s}$ in $0.5 \mathrm{~m} / \mathrm{s}$ increments and vertical control plane deflections from $2.5^{\circ}$ to $15.0^{\circ}$ in $2.5^{\circ}$ increments.

For the Baseline Configuration, the turning diameter does not change considerably with increasing forward velocity. For example, for a control plane deflection of $10^{\circ}$, the turning diameter ranges from $16.5 \mathrm{~m}$ at $0.5 \mathrm{~m} / \mathrm{s}$ to $16.7 \mathrm{~m}$ at $4 \mathrm{~m} / \mathrm{s}$. For the sake of brevity, results will only be presented for the case of $2 \mathrm{~m} / \mathrm{s}$. As expected, the turning diameter of the vehicle decreases with increasing control plane deflection (see Figure 3.6). The vehicle becomes unstable from $0.5 \mathrm{~m} / \mathrm{s}$ to $4.0 \mathrm{~m} / \mathrm{s}$ at a control plane deflection angle of $15^{\circ}$. This is due to the fact that once the vehicle enters the turn, the angle of attack on the vertical control planes increases, possibly exceeding the stall angle, thus reducing the stabilizing moment generated by the control planes (see Figure 3.7).

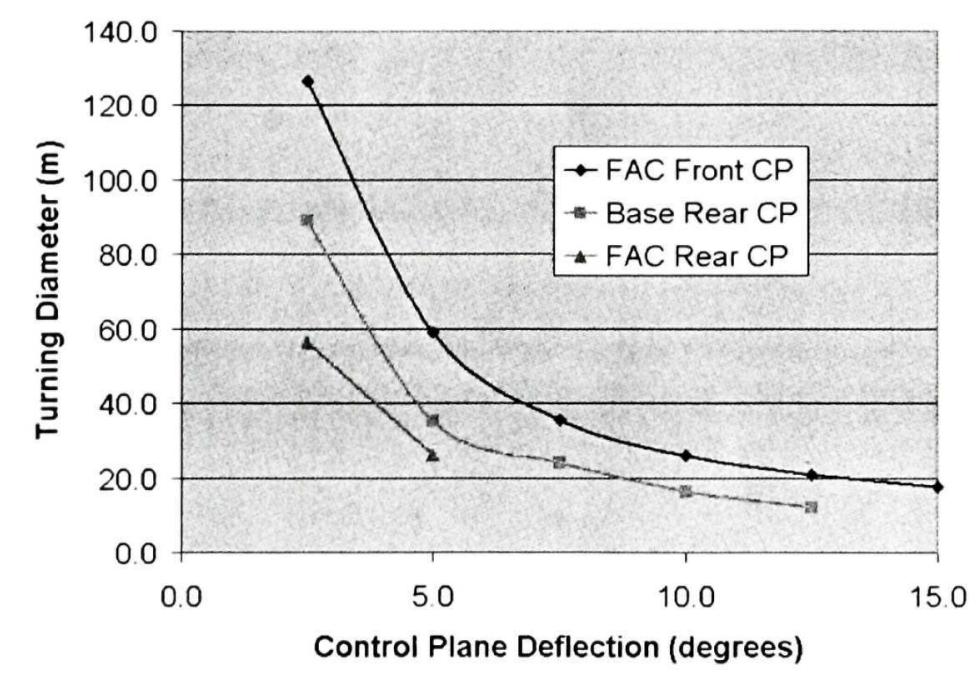

Figure 3.6 - Turning Diameters for C-SCOUT Baseline and Fully Actuated Configurations at $V=2.0 \mathrm{~m} / \mathrm{s}$ 


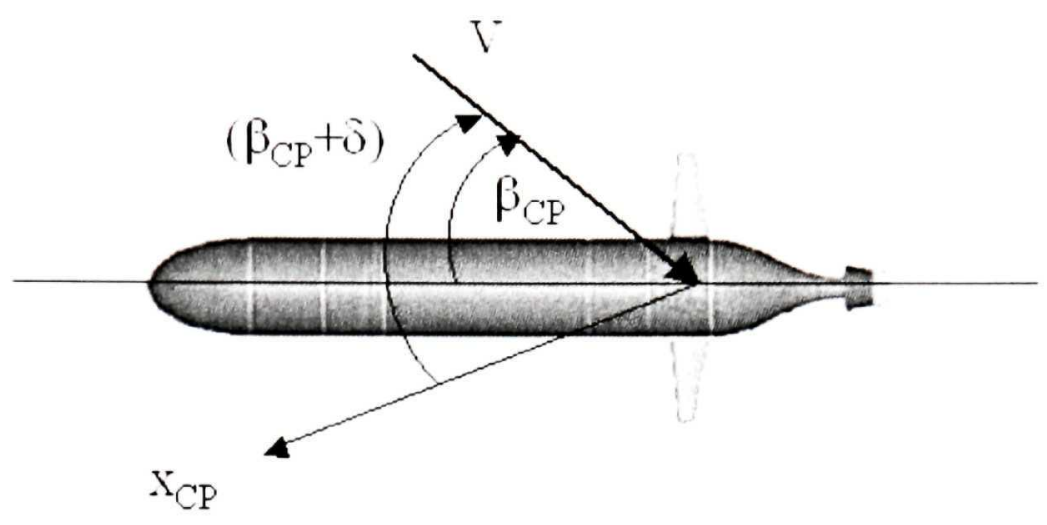

Figure 3.7 - Angle of Attack Increase on the Rear Control Planes in a Turn to the Right (Top View)

For the Fully Actuated Configuration, a rear control plane deflection of $7.5^{\circ}$ or more causes the vehicle to enter an unstable spiral. We also note that the smallest turn possible while remaining stable, using the rear control planes, is around $26 \mathrm{~m}$, compared to about $12 \mathrm{~m}$ for the Baseline Configuration (see Figure 3.6). If we instead use the forward control planes to turn the vehicle, we find it is more stable. This difference in stability between using the front or rear control planes is due to the direction in which the planes are deflected to turn the vehicle. When we use the rear control planes to turn, the control planes point away from the direction of the turn (see Figure 3.7), and the deflection angle is added to the sideslip angle to obtain the plane's angle of attack. However, when we use the forward control planes to turn the vehicle, the control planes point into the turn (as control planes are forward of the center of mass of the vehicle). In this case, the deflection angle is subtracted from the sideslip angle to form the angle of attack on the control planes. This reduces the angle of attack and hence the destabilizing moment. Additionally, the rear control planes are better able to stabilize the vehicle in this 
maneuver, as they will not stall unless the sideslip angle exceeds the stall angle. Thus we can achieve a turning diameter of $17.5 \mathrm{~m}$ with a deflection of $15^{\circ}$ of the forward control planes (see Figure 3.6).

The use of through-body thrusters in the Fully-Actuated Configuration was examined at forward velocities from $0.1 \mathrm{~m} / \mathrm{s}$ to $0.5 \mathrm{~m} / \mathrm{s}$ in $0.1 \mathrm{~m} / \mathrm{s}$ increments for thruster speeds of 200 RPM to 1000 RPM. The results showed that the use of the thrusters for much of this range reduced the vehicle's stability, especially at lower speeds. Also, the use of the rear thrusters in this range caused the vehicle to be more unstable than when the front thrusters were used. This difference is most likely caused by the asymmetry of the front and rear geometry of the vehicle.

An illustration of the effect of forward velocity and thruster speed on the turning diameter is shown in Figures 3.8 and 3.9, respectively. When using the through-body thrusters for turning the vehicle, we observe that, as the forward velocity increases, the turning diameter becomes quite large for low thruster speeds (see Figure 3.8). Increasing the thruster speed has the effect of significantly reducing the turning diameter (see Figure 3.9). However using high thruster speeds at low velocities can cause the vehicle to become unstable. Comparing the through-body thruster turning diameter to the control plane turning diameter at a forward velocity of $0.5 \mathrm{~m} / \mathrm{s}$, we see that, at high thruster speeds, the through-body thrusters have the capability to turn the vehicle more sharply than the control planes (13.2 $\mathrm{m}$ vs. $18.5 \mathrm{~m}$ using the front planes). 


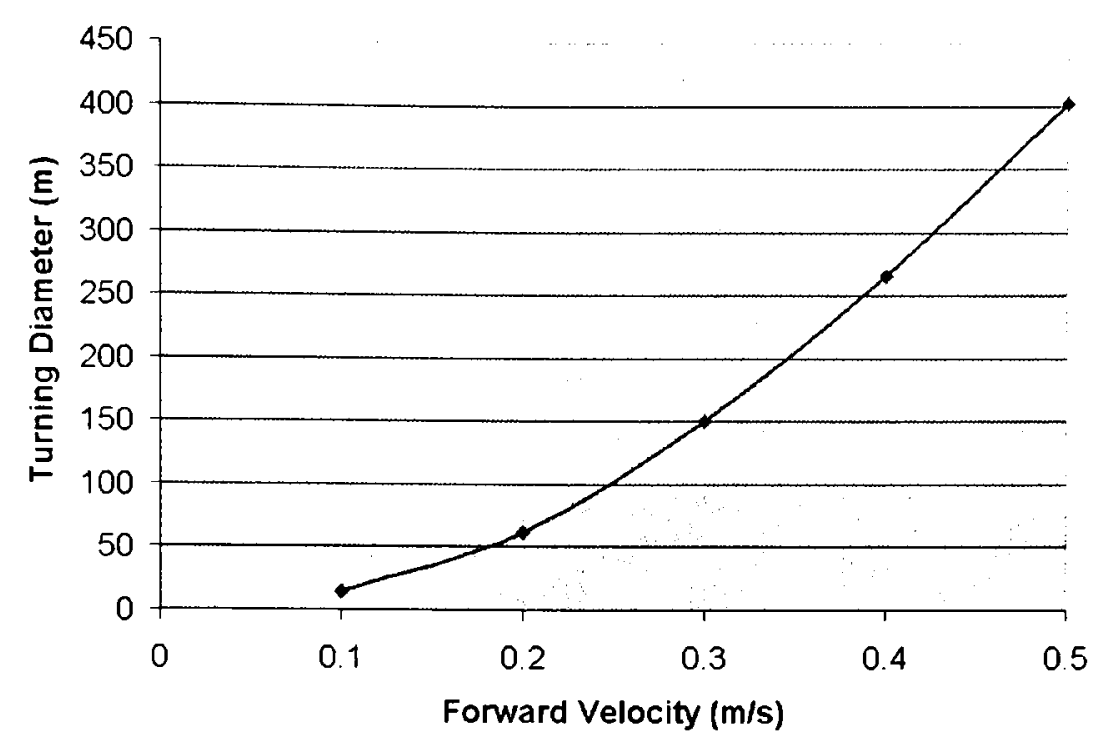

Figure 3.8 - Turning Diameter for Fully Actuated Configuration with Front ThroughBody Thrusters at 200 RPM

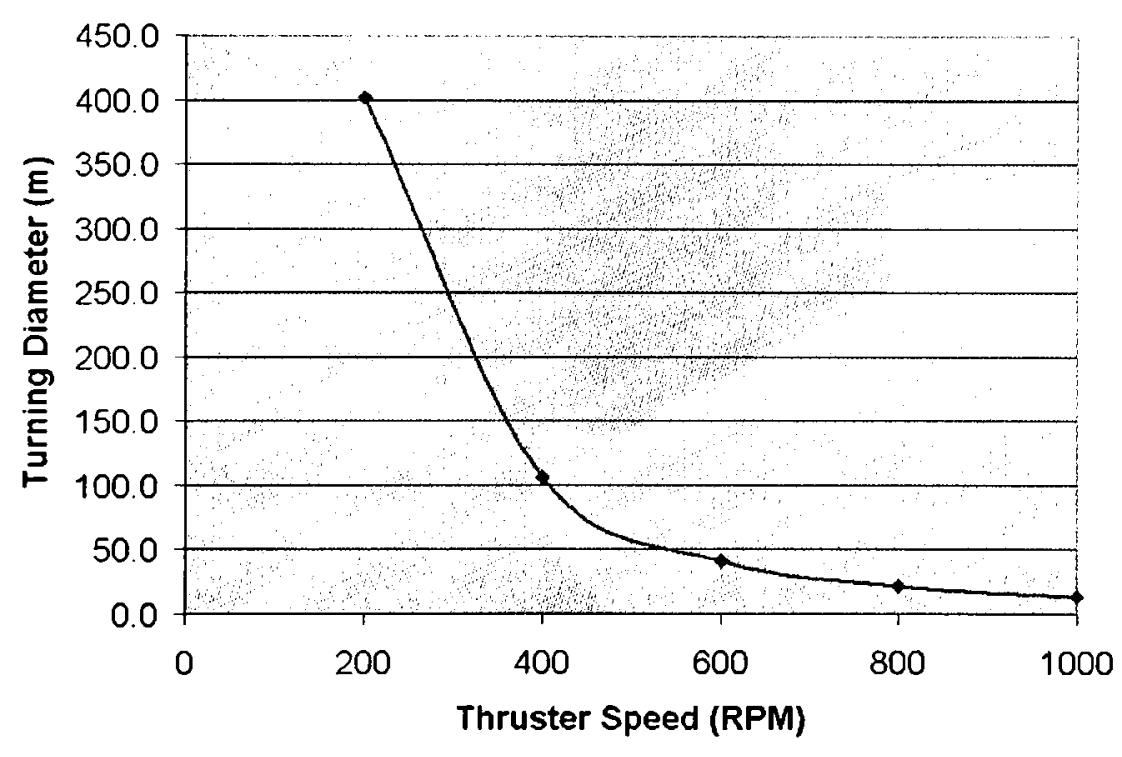

Figure 3.9 - Turning Diameter for Fully Actuated Configuration with Front ThroughBody Thrusters at $V=0.5 \mathrm{~m} / \mathrm{s}$ 


\section{Chapter 4 Mission Simulation}

C-SCOUT was designed to have the ability to conduct oceanographic sampling missions. We wish to compare the ability of the Baseline and Fully Actuated Configurations of C-SCOUT to successfully complete such a mission.

Typically oceanographic sampling missions are conducted in a 'mower' pattern, consisting of a series of straight tracks, which combine to form a rectangular sampling area. We will define a volume of $100 \mathrm{~m}$ by $100 \mathrm{~m}$ by $20 \mathrm{~m}$ depth in which we would like to sample. Specific points to sample inside this volume consist of 18 waypoints (see Figure 4.1), starting from an initial position of $(0,0,0)$. We adjust the depth of the waypoints along the tracks to illustrate the vehicle's ability to follow a more complex path than simply a planar survey. An example of where this may be useful is if the vehicle needs to follow the contours of the ocean floor. 


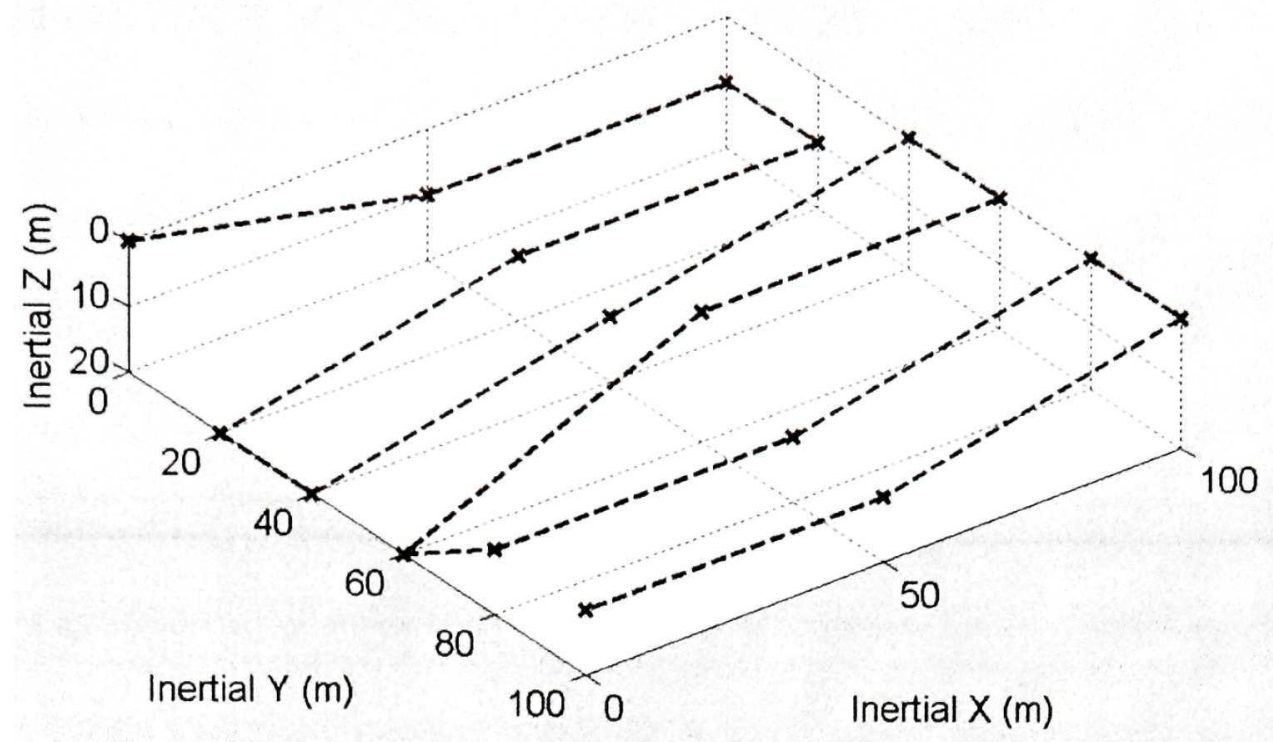

Figure 4.1 - Mission Sampling Volume and Waypoints

We will define two missions to be evaluated based on these waypoints: one where the vehicle traverses the waypoints without slowing down (continuous sampling), and another where the vehicle must stop at each waypoint to gather data (discrete sampling). We determine that the vehicle has reached a waypoint when it has come within some distance tolerance of that waypoint, which we will set as $2 \mathrm{~m}$. For the Discrete Sampling Mission, we will set a sampling time in which the vehicle must remain stationary at the waypoint of $20 \mathrm{~s}$. A situation can occur where the vehicle misses the waypoint on the first pass, and, based on the control system, attempts to loop around to reach the waypoint again. It is possible that the vehicle will continue looping around and never reach the waypoint; therefore we will limit the number of turns to 1.5 turns, or a heading angle change of $540^{\circ}$, before skipping the waypoint and targeting the next waypoint. 
We would also like to test C-SCOUT using another waypoint selection. It may be that we do not want to follow such an orderly waypoint path as that shown in Figure 4.1. Instead, we may want to traverse a more complicated path, possibly due to an optimization method that has chosen the waypoints, or a large number of physical obstructions. As we do not know what such a path would resemble, we will instead create a course with randomly generated waypoint coordinates. For convenience, we will use the same number of waypoints as before (18), the same sample volume $(100 \mathrm{~m}$ by $100 \mathrm{~m}$ by $20 \mathrm{~m})$, and set the first waypoint to be located at $(0,0,0)$. Using a random number generator to generate 18,3-number coordinates between 0 and 1 , and then scaling by 100,100 , and 20 for the inertial $\mathrm{X}, \mathrm{Y}$, and $\mathrm{Z}$ axes respectively, we obtain the waypoint course shown in Figure 4.2. We will traverse this course as we did in the Continuous Sampling Mission at an equilibrium velocity of $2 \mathrm{~m} / \mathrm{s}$, initially directed along the inertial $\mathrm{X}$ axis, starting at $(0,0,0)$.

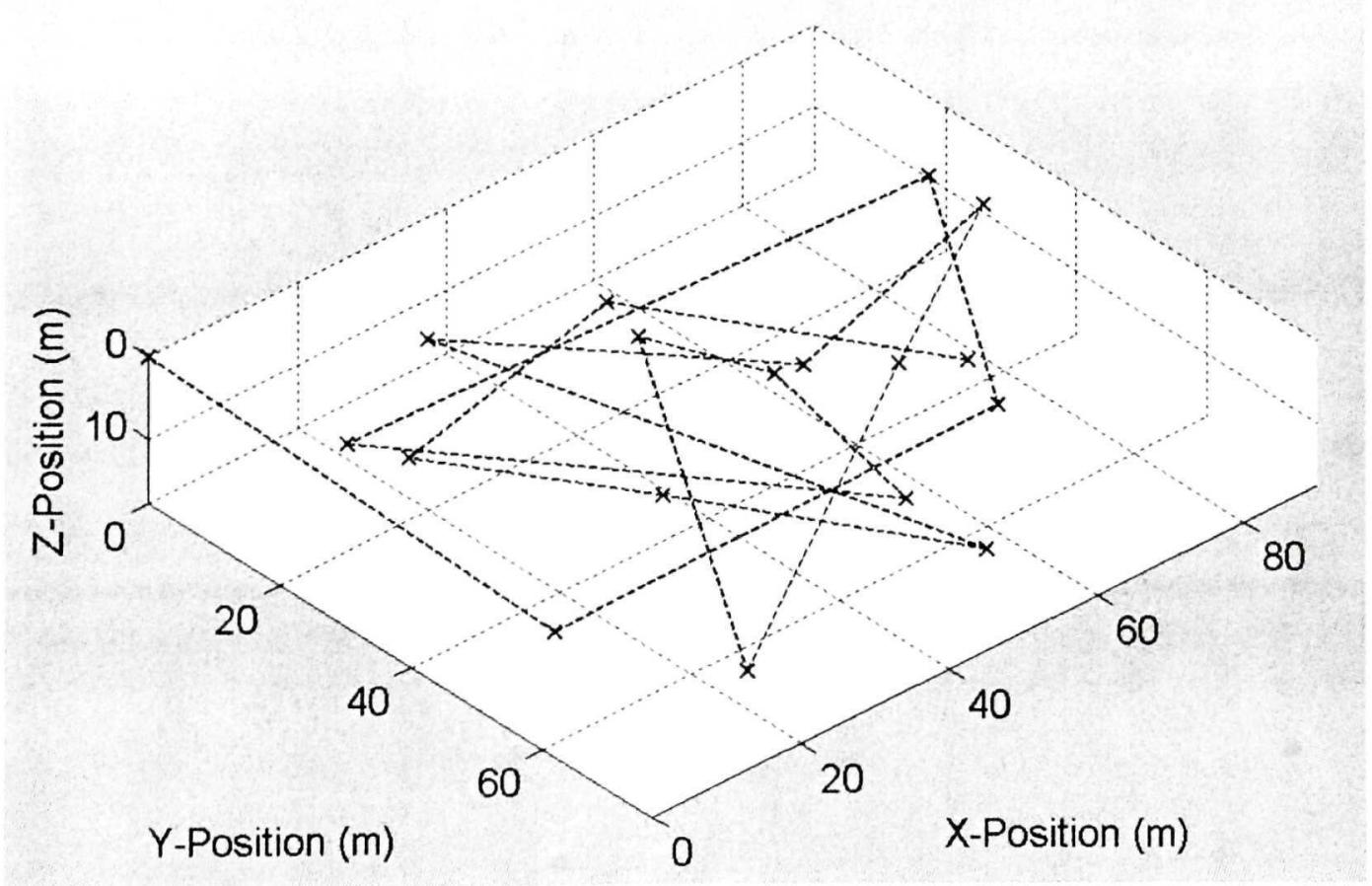

Figure 4.2 - Random Waypoint Selection 


\subsection{Controllers}

In order to give our vehicle simulation autonomous abilities for these missions, we must create a controller and a control strategy for the vehicle.

We define a mission path, which we would like the vehicle to follow, as being a straight line between the waypoints shown in Figures 4.1 and 4.2 . We wish to follow this path as closely as possible. We therefore design a controller, which we will call a 'cross-track' controller, which attempts to minimize the distance between the vehicle and the desired path.

We start by defining a new co-ordinate system, with its $\mathrm{x}$-axis pointing from the previous waypoint to the next waypoint that we wish to reach (see Figure 4.3). We can transform the vehicle's coordinates in the inertial frame to the coordinates in the new frame (designated by the subscript, $C T$ ) with the following homogeneous transformation matrix:

$$
\left[\begin{array}{c}
x_{C T} \\
y_{C T} \\
z_{C T} \\
1
\end{array}\right]=\mathbf{T}_{\mathrm{CT}} \cdot\left[\begin{array}{c}
X \\
Y \\
Z \\
1
\end{array}\right]
$$

where the transformation matrix, $\mathbf{T}_{\mathrm{CT}}$, is calculated as:

$$
\mathbf{T}_{\mathrm{CT}}=\mathbf{R}_{\mathrm{y}^{\prime}} \cdot \mathbf{R}_{\mathrm{Z}} \cdot \mathbf{T}_{\mathrm{x}}
$$

and $\mathbf{R}_{\mathrm{y}}, \mathbf{R}_{\mathrm{Z}}$, and $\mathbf{T}_{\mathrm{x}}$ are defined as: 


$$
\begin{aligned}
& \mathbf{R}_{Z}\left(\psi_{\text {way }}\right)=\left[\begin{array}{cccc}
c \psi_{\text {way }} & s \psi_{\text {way }} & 0 & 0 \\
-s \psi_{\text {way }} & c \psi_{\text {way }} & 0 & 0 \\
0 & 0 & 1 & 0 \\
0 & 0 & 0 & 1
\end{array}\right] \\
& \mathbf{R}_{y^{\prime}}\left(\theta_{\text {way }}\right)=\left[\begin{array}{cccc}
c \theta_{w a y} & 0 & -s \theta_{w a y} & 0 \\
0 & 1 & 0 & 0 \\
s \theta_{w a y y} & 0 & c \theta_{w a y} & 0 \\
0 & 0 & 0 & 1
\end{array}\right] \\
& \mathbf{T}_{x}=\left[\begin{array}{cccc}
1 & 0 & 0 & -X_{p n \cdot} \\
0 & 1 & 0 & -Y_{p r} \\
0 & 0 & 1 & -Z_{p r r} \\
0 & 0 & 0 & 1
\end{array}\right]
\end{aligned}
$$

and angles, $\theta_{w a y}$ and $\psi_{\text {way }}$ are defined as:

$$
\begin{gathered}
\psi_{w u y v}=\tan ^{-1}\left(\frac{\left(Y_{n x t}-Y_{p r r^{\prime}}\right)}{\left(X_{n x t}-X_{p r v}\right)}\right) \\
\theta_{w a y}=-\tan ^{-1}\left(\frac{\left(Z_{n x t}-Z_{p r v}\right)}{\sqrt{\left(X_{n x t}-X_{p r r}\right)^{2}+\left(Y_{n x t}-Y_{p r v}\right)^{2}}}\right)
\end{gathered}
$$

where the subscripts, prv and $n x t$ designate the previous and next waypoints, respectively, and $X_{i}, Y_{i}$, and $Z_{i}$ are the coordinates of waypoint $i$ in the inertial frame. 


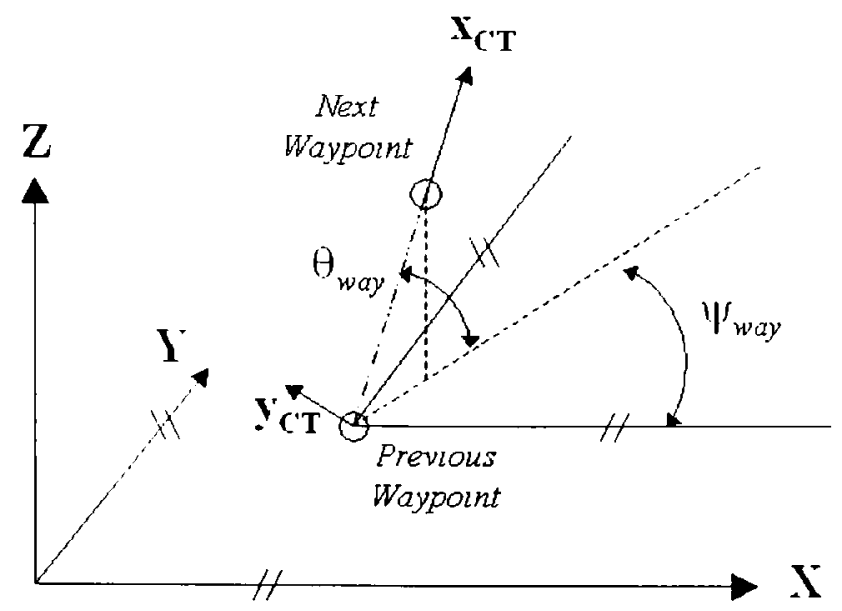

Figure 4.3-Controller Frame Transformation Angles

The cross-track controller directs the vehicle to a target point. This target point lies along the straight line between the waypoints, a distance $\Delta=10 \mathrm{~m}$ ahead of the vehicle (see Figure 4.4 ). The target position in the inertial frame can then be calculated as follows:

$$
\left[\begin{array}{c}
X_{t a r} \\
Y_{t a r} \\
Z_{a r} \\
1
\end{array}\right]=\mathbf{T}_{\mathrm{R}}^{-1} \cdot\left[\begin{array}{c}
x_{{ }^{\prime} T}+\Delta \\
0 \\
0 \\
1
\end{array}\right]
$$

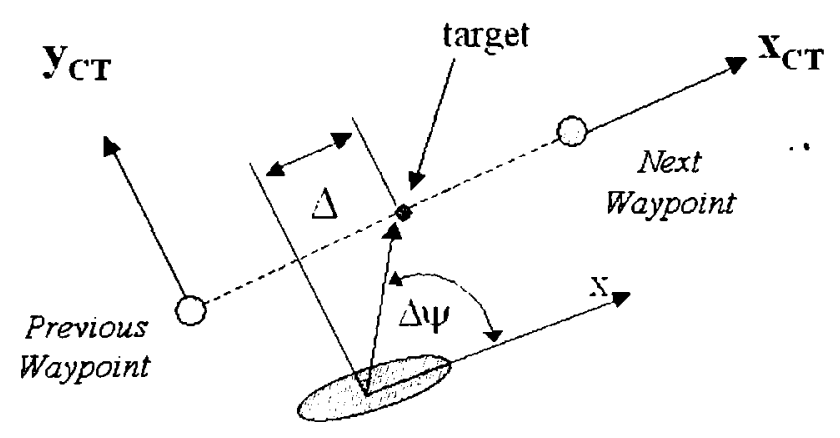

Figure 4.4 - Target Point for the Cross-Track Controller 
Once the target point has been determined, we can use a proportional controller to adjust the control planes to direct the vehicle towards it. Additionally we wish to actively control the vehicle's roll angle, maintaining it as close as possible to zero. We will limit the control plane deflections to $\pm 10^{\circ}$ for all four of the control planes. For the Baseline Configuration, the control plane deflections are as follows:

$$
\begin{aligned}
& \left(\delta_{\text {rear }}\right)_{\text {top }}=K_{h} \Delta \psi-K_{a} \phi \\
& \left(\delta_{\text {rear }}\right)_{\text {tot }}=K_{h} \Delta \psi+K_{u} \phi \\
& \left(\delta_{\text {rear }}\right)_{p r t}=K_{v} \Delta \theta-K_{a} \phi \\
& \left(\delta_{\text {rear }}\right)_{s t h}=K_{v} \Delta \theta+K_{u} \phi
\end{aligned}
$$

where $\delta$ is the deflection of the appropriate control plane, the subscripts for, rear, top, bot, prt, and stb designate the forward, rear, upper, lower, port, and starboard control planes, respectively, $K_{h}, K_{v}$, and $K_{a}$ are the horizontal heading gain, vertical heading gain, and roll gain, respectively, and $\Delta \psi$ and $\Delta \theta$ are the horizontal angle error and the vertical angle error, respectively, which are calculated as:

$$
\begin{gathered}
\Delta \psi=-\tan ^{-1}\left(\frac{Y_{\text {tar }}-Y}{X_{\text {tar }}-X}\right)+\psi \\
\Delta \theta=\tan ^{-1}\left(\frac{Z_{t a r}-Z}{\sqrt{\left(X_{t a r}-X\right)^{2}+\left(Y_{t a r}-Y\right)^{2}}}\right)+\theta
\end{gathered}
$$

For the Fully Actuated Configuration, we have an additional control plane module forward of the center of mass. In Section 3.3, we found that turning the vehicle using the 
forward control planes made for a more stable vehicle. Therefore, we choose to use the forward vertical control planes for yaw control. We will use the rear horizontal control planes for depth control, as rapid changes in depth are not required, and therefore an unstable situation is unlikely to occur. We will use all four rear control planes for roll control. In our simulations the forward horizontal control planes are not active, but they could be used for pitch control, as a large pitch angle could be undesirable. We will limit the rear control planes to $\pm 10^{\circ}$, as was done on the Baseline Configuration, and the front control planes to $\pm 15^{\circ}$, as a higher control plane deflection is required to obtain the same turning diameter as the Baseline Configuration. The control plane deflections for the Fully Actuated Configuration are described as follows:

$$
\begin{gathered}
\left(\delta_{\text {for }}\right)_{u r p}=-K_{h} \Delta \psi \\
\left(\delta_{\text {for }}\right)_{\text {bot }}=-K_{h} \Delta \psi \\
\left(\delta_{\text {for }}\right)_{p r t}=0 \\
\left(\delta_{\text {for }}\right)_{s t b}=0 \\
\left(\delta_{\text {rear }}\right)_{t o p}=-K_{a} \phi \\
\left(\delta_{\text {rear }}\right)_{b o t}=K_{a} \phi \\
\left(\delta_{\text {rear }}\right)_{p r t}=K_{v} \Delta \theta-K_{a} \phi \\
\left(\delta_{\text {rear }}\right)_{s t b}=K_{v} \Delta \theta+K_{a} \phi
\end{gathered}
$$


For the Continuous Sampling mission, rear thruster control is achieved with (65) and control plane deflections are determined by (92) or (95), depending on the vehicle configuration.

For the Discrete Sampling mission we also need to control the speed of the vehicle in order to stop at each waypoint. To accomplish this we define a set of tactics for the vehicle to follow:

- Transit - Traverse the distance between waypoints.

- Approach - Slow the vehicle to a stop at the waypoint.

- Capture - Remain stationary at the waypoint for a set period of time.

- Reorient - (Fully Actuated Configuration only) Use through-body thrusters to reorient the vehicle towards the next waypoint.

The tactics, 'Transit' and 'Approach' both use the control plane deflections listed in (92) and (95) for the Baseline and Fully Actuated Configurations, respectively. For 'Transit', the thrust from the rear thruster is maintained at the value determined by $(65)$. For 'Approach', the thrust from the rear thruster is determined by the following relationship, which will cause the vehicle to slow to a stop at the next waypoint:

$$
T=-K_{T u} u+K_{T x} d_{u p p}
$$

where $K_{T u}$ and $K_{T x}$ are velocity and distance gains, respectively, and $d_{w p}$ is the distance to the next waypoint. The values used for the gains described in equations (92), (95), and (96) are listed in Table 4.1: 


\begin{tabular}{|c|c|c|c|}
\hline Gain Variable & Baseline Configuration & Fully Actuated Configuration & Units \\
\hline$K_{h}$ & 0.5 & 0.2 & $\mathrm{rad} / \mathrm{rad}$ \\
\hline$K_{v}$ & 0.25 & 0.25 & $\mathrm{rad} / \mathrm{rad}$ \\
\hline$K_{a}$ & 0.5 & 0.5 & $\mathrm{rad} / \mathrm{rad}$ \\
\hline$K_{T u}$ & 100 & 100 & $\mathrm{Ns} / \mathrm{m}$ \\
\hline$K_{T x}$ & 5 & 5 & $\mathrm{~N} / \mathrm{m}$ \\
\hline
\end{tabular}

Table 4.1 - Controller Gains

The tactic, 'Capture' is initiated when the vehicle is deemed to have reached the waypoint. At this point the vehicle has stopped, and the control plane deflections are set to zero. The rear thruster and, if available, the through-body thrusters are used to maintain a stationary position. At this point the vehicle would begin sampling the surrounding water. Once the sampling time has expired, the vehicle will attempt to reach the next waypoint. For the Baseline Configuration, the vehicle returns to the 'Transit' tactic. For the Fully Actuated Configuration, the vehicle initiates the 'Reorient' tactic and uses the through-body thrusters to orient the vehicle towards the next waypoint. Once the vehicle is directed at the waypoint, the 'Transit' tactic is initiated.

\subsection{Mission Performance Evaluation}

For the Continuous Sampling mission, the Baseline Configuration does a good job of reaching each waypoint (see Figure 4.5) - only one waypoint is not reached at first attempt. However, that waypoint is reached after looping around. At an equilibrium velocity of $2.0 \mathrm{~m} / \mathrm{s}$, the Baseline Configuration completes the Continuous sampling mission in $421.0 \mathrm{~s}$. 


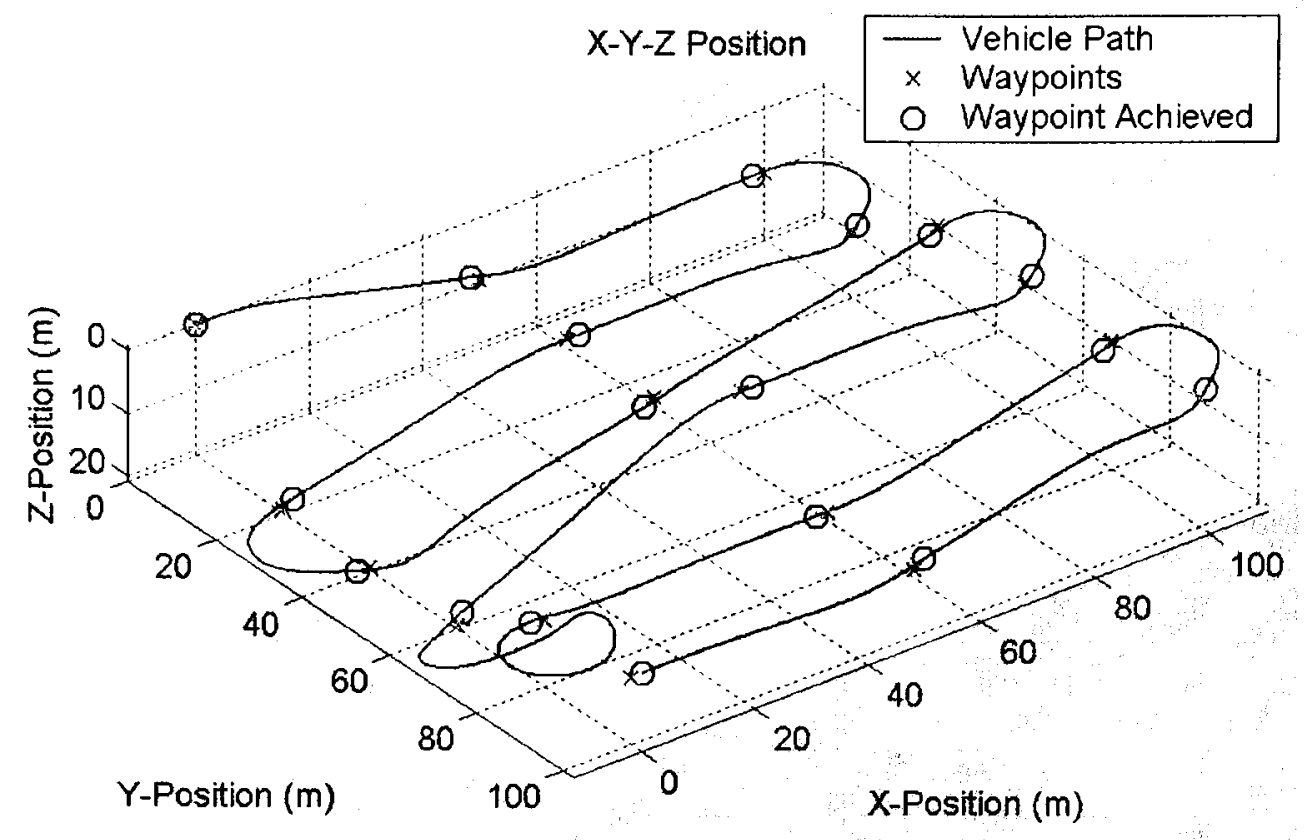

Figure 4.5-C-SCOUT Baseline Configuration: Continuous Sampling Mission, Inertial $X-Y-Z$ Plot

The Fully Actuated Configuration has more difficulty than the Baseline Configuration in following the Continuous Sampling mission (see Figure 4.6). In particular, when the vehicle attempts to make a sharp turn, it tends to turn more quickly than desired and miss the next waypoint. The vehicle is able to recover, but is then unable to reach the waypoint. At an equilibrium velocity of $2.0 \mathrm{~m} / \mathrm{s}$, the Fully Actuated Configuration partially completes the Continuous Sampling mission, reaching only 13 out of 18 waypoints, in $724.9 \mathrm{~s}$. 


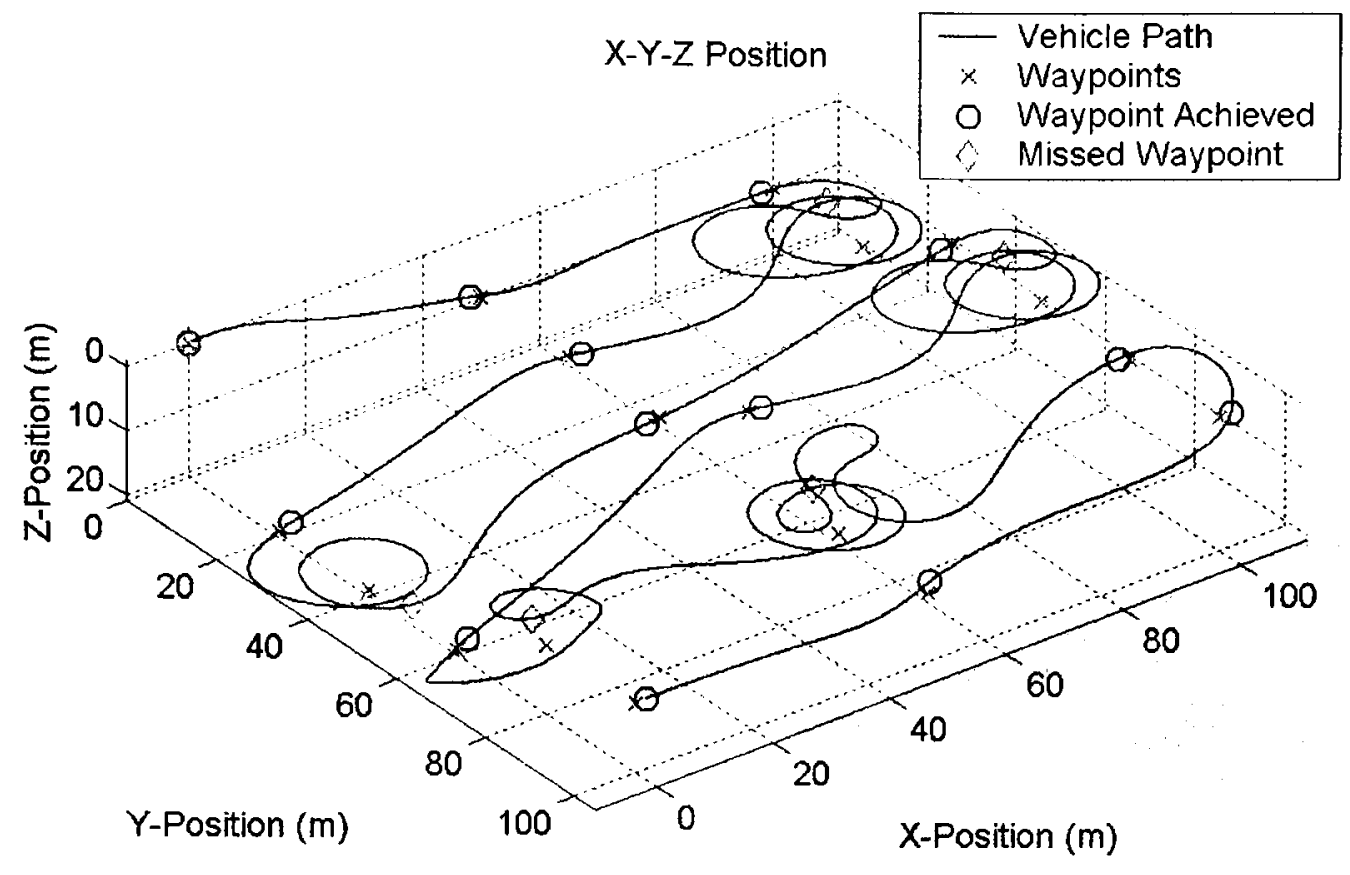

Figure 4.6-C-SCOUT Fully Actuated Configuration: Continuous Sampling Mission. Inertial $X-Y-Z$ Plot

For the Discrete Sampling mission, the vehicle stops at each waypoint and remains stationary for the duration of the set sampling time, which we have chosen to be $20 \mathrm{~s}$. For this mission, the Baseline Configuration exhibits some unstable characteristics when the vehicle attempts to make a $90^{\circ}$ turn from a zero velocity (see Figure 4.7). At an equilibrium velocity of $2.0 \mathrm{~m} / \mathrm{s}$, the Baseline Configuration partially completes the Discrete Sampling mission, reaching 17 out of 18 waypoints, in 1227.0 s. 


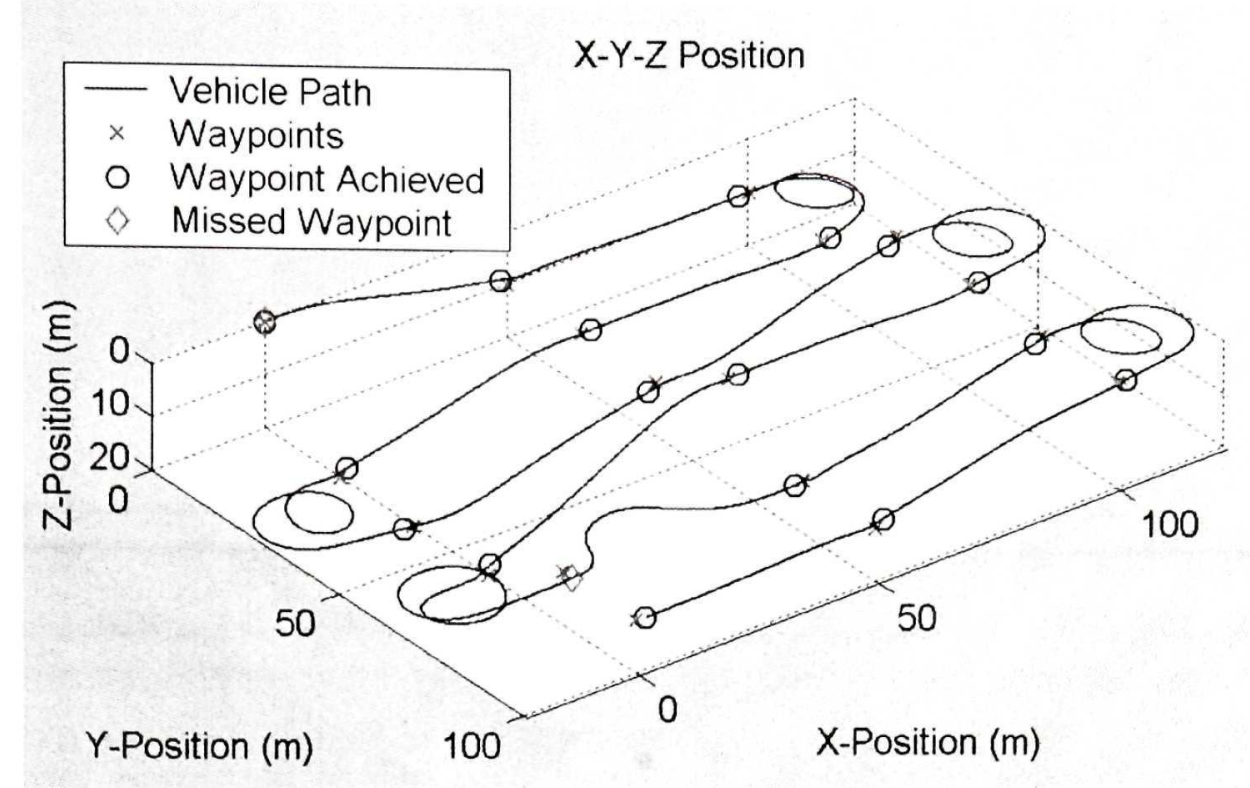

Figure 4.7 - C-SCOUT Baseline Configuration: Discrete Sampling Mission, Inertial XY-Z Plot

The Fully Actuated Configuration performs about the same as the Baseline Configuration in the Discrete Sampling mission. The use of the through-body thrusters enables the vehicle to orient itself directly towards the next waypoint (see Figure 4.8). At an equilibrium velocity of $2.0 \mathrm{~m} / \mathrm{s}$, the Fully Actuated Configuration partially completes the Discrete Sampling mission, reaching 17 out of 18 waypoints, in $1587.2 \mathrm{~s}$. We note that for both configurations in each mission, the vehicle has difficulty attaining the $13^{\text {th }}$ waypoint. This is due to the sharp change in pitch that is required from the $12^{\text {th }}$ to the $13^{\text {th }}$ waypoint. The vehicle will be pitched down when it travels from the $11^{\text {th }}$ to the $12^{\text {th }}$ waypoint, and then must pitch up to achieve the $13^{\text {th }}$ waypoint, which lies $10 \mathrm{~m}$ above and $20 \mathrm{~m}$ beside the $12^{\text {th }}$ waypoint. 


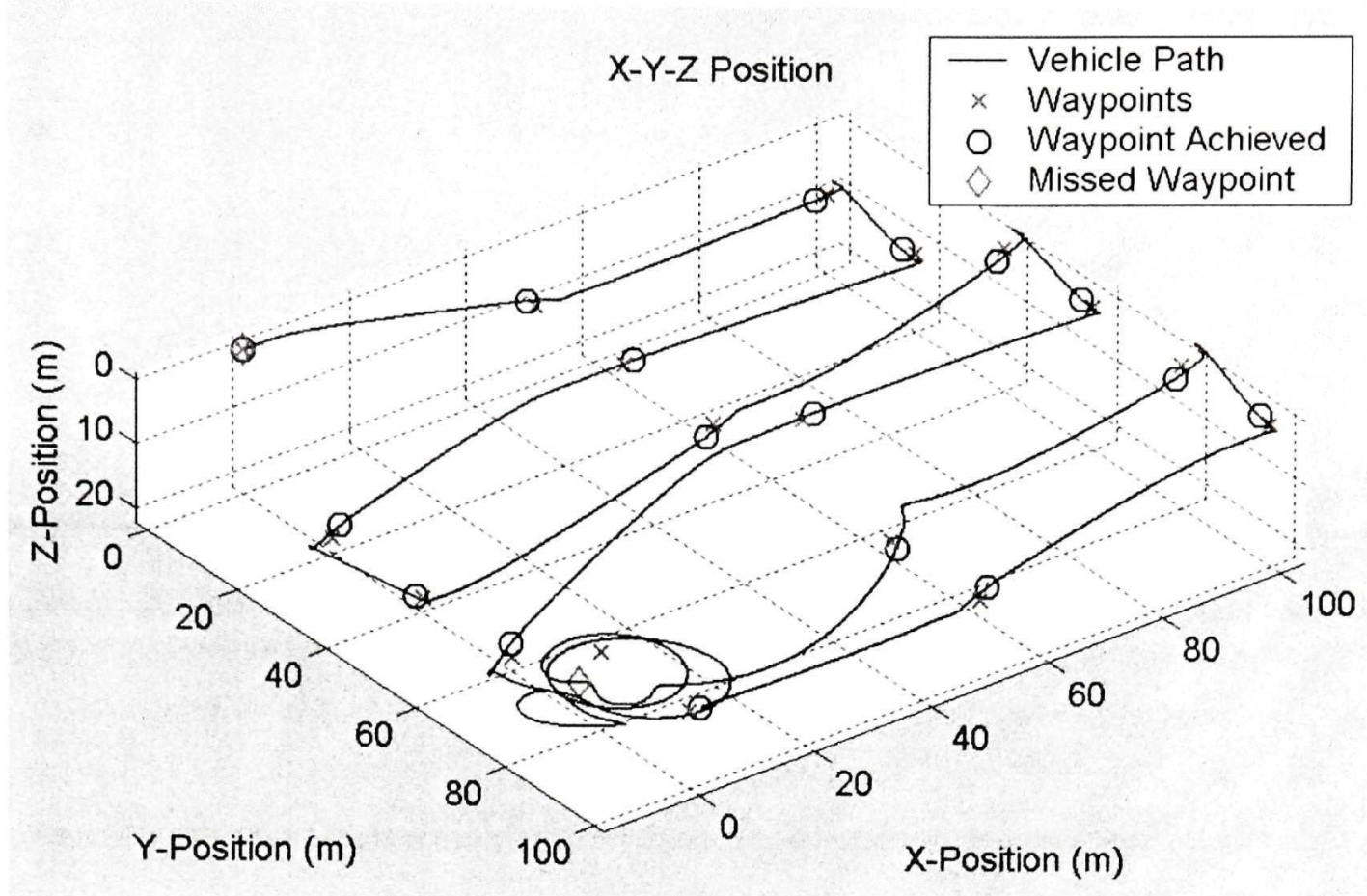

Figure 4.8 - C-SCOUT Fully Actuated Configuration: Discrete Sampling Mission, Inertial $X-Y-Z$ Plot

We now examine the randomly generated waypoint course. Despite the apparent difficulty in this course, due to the sharp turns that are required, the Baseline Configuration does quite well in completing the mission (see Figure 4.9). Only one waypoint is not achieved and no instabilities are observed. The Baseline Configuration completes the mission in $547.0 \mathrm{~s}$. 


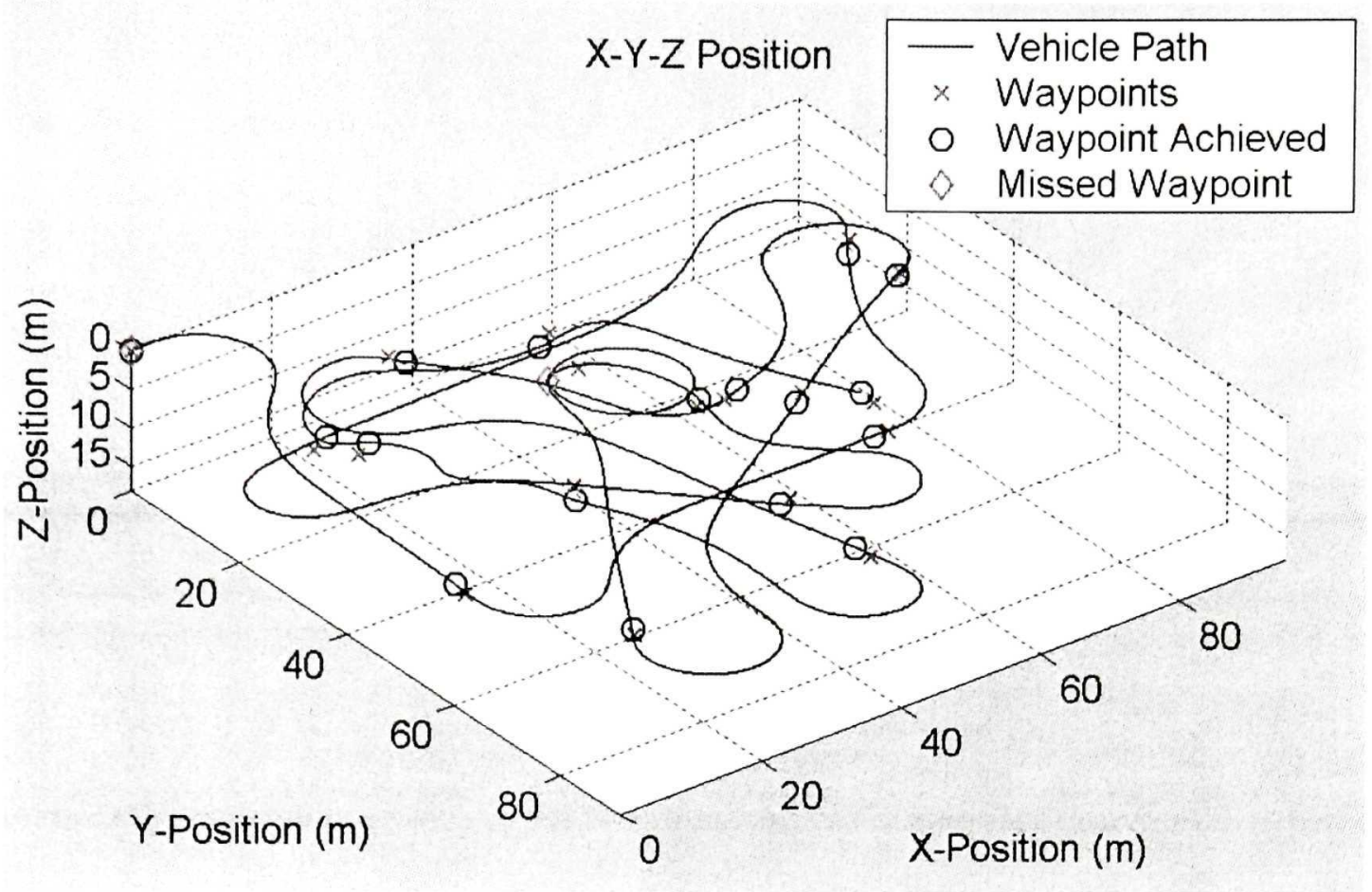

Figure 4.9-C-SCOUT Baseline Configuration: Randomly Generated Waypoints, Inertial $X-Y-Z$ Plot

For the Fully Actuated Configuration, we again observe difficulties when a sharp turn is initiated (see Figure 4.10). This causes overshoot of the required angle change and causes the vehicle to perform poorly in following the waypoint path. The Fully Actuated Configuration reaches 15 out of 18 waypoints in $957.8 \mathrm{~s}$. 


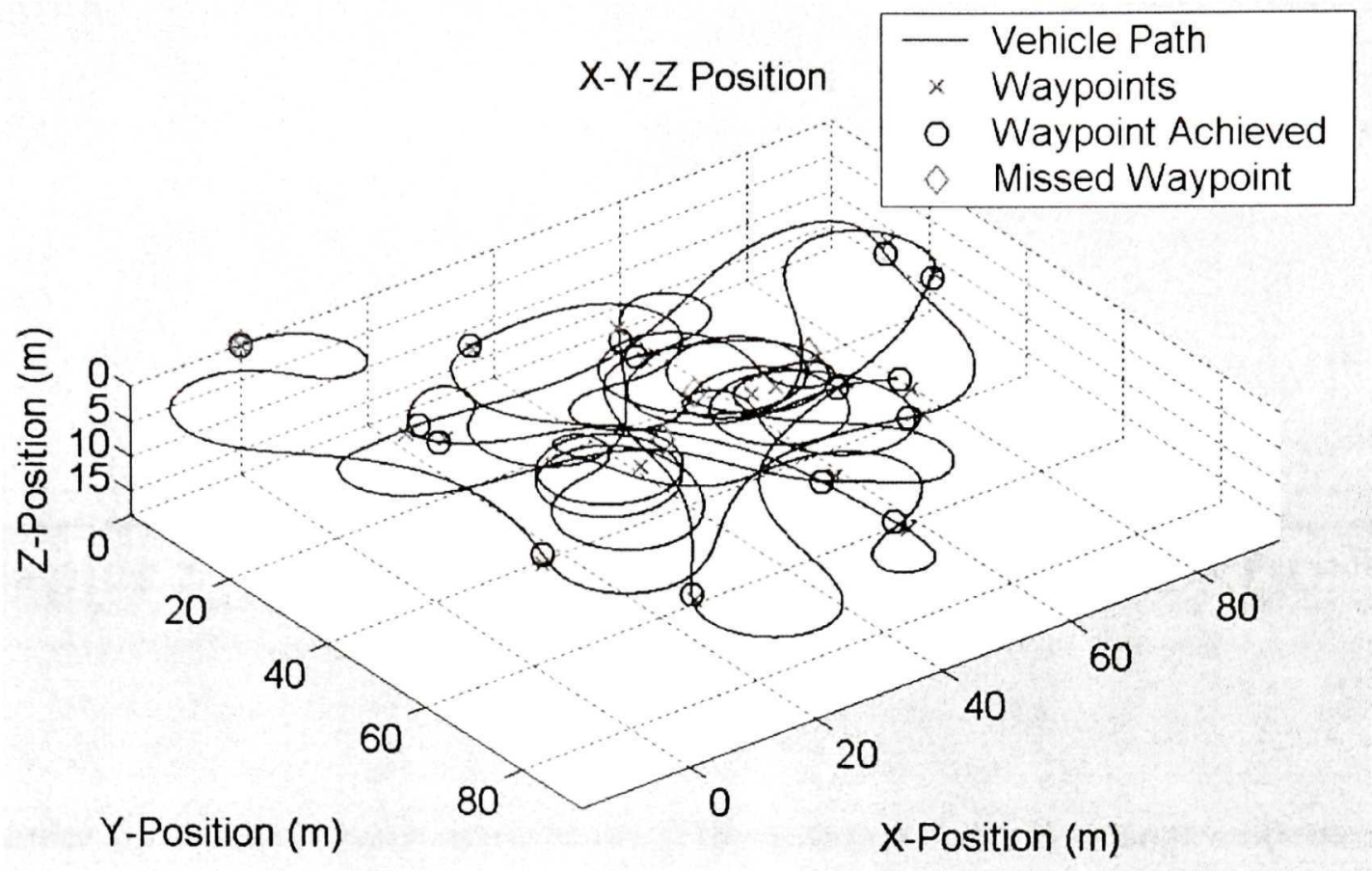

Figure 4.10-C-SCOUT Fully Actuated Configuration: Randomly Generated Waypoints, Inertial $X-Y-Z$ Plot 


\section{Chapter 5 CONCLUSIONS AND RECOMMENDATIONS}

An underwater vehicle, such as an AUV, is likely to encounter high angles of attack on the hull and control planes during the course of a mission. This can occur at low vehicle speeds or in the presence of a cross-flow. The use of Jorgensen's [13] method enables us to predict the hull forces and moments for the full angle of attack range. We are also able to model the forces on the control planes for the full angle of attack range using lift and drag coefficient data provided by Critzos [21]. The vehicle model was validated using field data of the turning diameters of the ARCS vehicle. Results showed that the static formulation of the hull forces more closely predicted the turning diameters for the vehicle than the dynamic formulation of the hull forces. As validation data is not currently available for the C-SCOUT vehicle, we assume that the static formulation will also provide a more accurate prediction of the forces on the C-SCOUT hull.

Saunders [8] showed his through-body thruster model to be quite accurate in the prediction of thruster performance on the C-SCOUT vehicle. In our simulation we have assumed that the performance of the thruster will be the same irrespective of the position 
and orientation of the thruster on of the hull. In reality, the fluid flow around the vehicle will change along the length of the hull, thus altering the values of $a$ and $b$ that should be used in the thruster model.

The Baseline and Fully Actuated Configurations of C-SCOUT are simulated to compare the predicted performance of the two configurations. For zero control plane deflections, the Baseline Configuration is more stable than the Fully Actuated Configuration. This is due to the destabilizing force generated by the forward control planes on the Fully Actuated Configuration. The stability of the Baseline Configuration decreases with increasing speed, while the Fully Actuated Configuration becomes unstable at about the same sideslip angle, irrespective of vehicle speed. When using the control planes, the Baseline Configuration remains stable except at large control plane deflections. Thus, limiting those deflections during maneuvering can improve the vehicle stability. For the Fully Actuated Configuration, using the front control planes to turn the vehicle can improve vehicle stability, as the destabilizing moment they generate is reduced due to the decreased angle of attack on the front control planes. The through-body thrusters in the Fully Actuated Configuration reduce vehicle stability if the vehicle is in motion, especially if high thruster speeds are used at low forward velocities. In all cases, it is expected that active control could enhance the vehicle stability.

Three simulated oceanographic sampling missions and a set of controllers and control tactics are created to determine circumstances where one configuration of C-SCOUT would be beneficial over the other. We find that the Baseline Configuration is suitable to 
missions where the vehicle maintains a forward speed. The Fully Actuated Configuration is found to be suitable to missions where it can use its through-body thrusters to change direction once it has stopped. Although it was not studied in this paper, it is expected that the Fully Actuated Configuration would show superior performance in the presence of cross-currents.

Recommendations for vehicle operation based on the performance results:

- Arrange the interior of the vehicle such that the center of mass and the center of buoyancy lie as far forward as possible. This will increase the stabilizing moment of the rear control planes, and reduce the destabilizing moment of the hull and, for the case of the Fully Actuated Configuration, the forward control planes.

- Limit the deflection of the rear control planes to angles less than the stall angle in order to increase vehicle stability.

- For the Fully Actuated Configuration, use the forward control planes for heading changes in order to increase vehicle stability.

- Do not use the through-body thrusters while the vehicle is in motion, as this will increase vehicle instability.

Recommendations for future work and for improving the simulation:

- Validate the predicted performance of the Baseline Configuration using experimental data.

- Incorporate the simulation into a mission planner and graphical user interface.

- Improve the intelligence of the controllers. 
- Define more specific and realistic missions in which to evaluate the performance of the two configurations.

- Recode the simulation in $\mathrm{C} / \mathrm{C}++$ in order to decrease the time required to run a mission simulation.

- Incorporate interference effects from the hull on the control planes depending on the velocity and orientation of the vehicle to the fluid flow. 


\section{REFERENCES}

1. Marco, D., Healey, A., Current Developments in Underwater Vehicle Control and Navigation: The NPS ARIES AUV, Oceans 2000, 2000, Vol. 2, pp. 1011 1016.

2. Whitney, J., Smith, S., Observations on the Dynamic Performance of Tunnel Thrusters, Oceans ‘98, 1998, Vol. 2, pp. 676-681.

3. Wang, H., Marks, R., McLain, T., Fleischer, S., Miles, D., Sapilewski, G.,Rock, S., Otter: A Testbed Submersible for Robotics Research, Proceedings of ANS 95, 1995.

4. Mailfert, G., LeMaire, J., REDERMOR: an Experimental platform for ROV/AUV Field Sea Trials, Oceans ‘98, 1998, Vol. 1, pp. 357-362.

5. Curtis, T., Perrault, D., Williams, C., Bose, N., C-SCOUT: A General-Purpose $A U V$ for Systems Research, Underwater Technology 2000, 2000, pp. 73-77.

6. Nahon, M., A Simplified Dynamics Model for Autonomous Underwater Vehicles, Autonomous Underwater Vehicle Technology AUV '96, 1996, pp. 373-379.

7. Perrault, D., Autonomous Underwater Vehicles (AUV) Sensitivity of Motion Response to Geometric and Hydrodynamic Parameters and AUV Behaviors with Control Plane Faults, Doctoral Thesis, Memorial University of Newfoundland, St. John's, Newfoundland, Canada, 2002.

8. Saunders, A., The Effect of Velocity and Orientation on the Simulation and Experimental Characterization of an AUV Tunnel Thruster, Masters Thesis, University of Victoria, Victoria, British Columbia, Canada, 2003.

9. Munk, M., The Aerodynamic Forces on Airship Hulls, Report No. 184, National Advisory Committee on Aeronautics (NACA), 1924.

10. Allen, H., Perkins, E., A Study of Effects of Viscosity on Flow Over Slender Inclined Bodies of Revolution, Report No. 1048, National Advisory Committee on Aeronautics (NACA), 1951.

11 Allen, H., Perkins, E., Characteristics of Flow Over Inclined Bodies of Revolution, Research Memorandum RM A50L07, National Advisory Committee on Aeronautics (NACA), 1951.

12. Hopkins, E., A Semiempirical Method for Calculating the Pitching Moment of Bodies of Revolution at Low Mach Numbers, Research Memorandum RM A51C14, National Advisory Committee on Aeronautics (NACA), 1951. 
13. Jorgensen, L., Prediction of Static Aerodynamic (haracteristic for SpaceShuttle-Like and Other Bodies at Angles of Attack from $0^{\circ}$ to $180^{\circ}$. NASA TN D6996, January 1973.

14. Polhamus, E., A Concept of Vortex Lift of Sharp-Edge Delta Wings Based on Leading-Edge-Suction Analogy, NACA TN D-3767, 1966.

15. Kelly, H., The Estimation of Normal-Force, Drag, and Pitching-Moment Coefficients for Blunt-Based Bodies of Revolution at Large Angles of Attack, Journal of Aerospace Science, 1954.

16. Ward, G., Supersonic Flow Past Slender Pointed Bodies, Quarterly Journal of Mechanics and Applied Mathematics, Vol 2, Part I, March 1949, pp. 75-97.

17. Finck, R., USAF Stability and Control DATCOM, McDonald Douglas Corp., Global Engineering Documents, 1978.

18. Jones, S. P., DeLaurier, J. D., Aerodynamic Estimation Techniques for Aerostats and Airships, Journal of A ircraft, V. 20, No. 2, 1982, pp. 120-126.

19. Abbott, I. H., Von Doenhoff, A. E., Theory of Wing Sections, Dover Publications, 1959.

20. Reigels, F. W., Aerodynamische Profile, Muchen, R. Oldenbourg, 1958.

21. Critzos, C. C., Heyson, H. H., Boswinkle Jr., R. W., Aerodynamic Characteristics of NACA 0012 Airfoil Section at Angles of Attack From $0^{\circ}$ to $180^{\circ}$, NACA TN $3361,1955$.

22. McCormick, B. W., Aerodynamics, Aeronautics, and Flight Mechanics, New York, Wiley, 1995.

23. Hopkin, D., Hertog, V. den, The Hydrodynamic Testing and Simulation of an Autonomous Underwater Vehicle, Proceedings of the Second Canadian Marine Dynamics Conference, pp. 274-281.

24. R. Thomas, Performance Evaluation of the Propulsion System of the Autonomous Underwater Vehicle C-SCOUT, Master's Thesis (in preparation), Memorial University of Newfoundland, St. John's, Newfoundland, Canada, 2003.

25. Pennel, V., Williams, C. D., Bose, N., Veitch, B., Hawbolt, K., Curtis, T., L., Perrault, D., E., O'Young, S., Mukhtasor, Sadiq, R., Husain, T., Ferguson, J., Eaton, G., Reedeker, P., Innovative Approaches to Environmental Effects Monitoring Using an Autonomous Underwater Vehicle, International Workshop 
on Underwater Robotics for Sea Exploitation and Environmental Monitoring, Rio de Janeiro, Brazil, October 1 to 3, 2001.

26. Kim, K., Sutoh, T., Ura, T., Obara, T., Route Keeping Control of AUV under Current by Using Dynamics Model Via CFD Analysis, OCEANS, 2001, Vol. 1, pp. 417-422.

27. Encarnacao, P., Pascoal, A., 3D Path Following for Autonomous Underwater Vehicle, Proceedings of the $39^{\text {th }}$ IEEE Conferenceon Decision and Control, Dec. 2000, Vol. 3, pp. 2977-2982.

28. Cristi, R., Papoulias, F., A., Healey, A., J., Adaptive Sliding Mode Control of Autonomous Underwater Vehicles in the Dive Plane, IEEE Journal of Oceanic Engineering, 1990, Vol. 15, No. 3, pp. 152-160.

29. Prestero, T., Development of a Six-Degree of Freedom Simulation Model for the REMUS Autonomous Underwater Vehicle, OCEANS, 2001, Vol. 1, pp. 450-455.

30. Kobayashi, E., Aoki, T., Maeda, T., Hirokawa, K., Ichikawa, T., Saitou, T., Miyamoto, S., Iwasaki, S., Kobayashi, H., Development of an Autonomous Underwater Vehicle Maneuvering Simulator, OCEANS, 2001, Vol. 1, pp. 361368.

31. Ferguson, J. and Jackson, E., Design of ARCS-Autonomous Remotely Controlled Submersible, ROV 1984 Conference Proceedings, Apr 1984, pp 365-368.

32. Humphreys, D., E., Smith, N., S., Hydrodynamics and Control of a Streamlined UUV Operating at 180-Degree Angle of Attack, $7^{\text {th }}$ International Symposium on Unmanned Untethered Submersible Technology, 1991, pp. 171-190. 


\section{APPENDIX A - DERIVATION OF DYNAMIC}

\section{Formulation OF Hull ForCES}

In this Appendix we derive a dynamic formulation of the hull forces on a streamlined body of rotation. This derivation follows the method presented by Jones and DeLaurier [18], but is based on static formulation of hull forces presented by Jorgensen [13] and includes an added mass factor as described in Section 2.2.2.1. Variable definitions are listed in the glossary.

We start by defining the normal force coefficient on the entire hull. Based on (28) in Section 2.2.2.1. The normal force coefficient is

$$
C_{N}=\left(k_{2}-k_{1}\right) \frac{S_{b}}{A} \sin (2 \alpha) \cos \frac{\alpha}{2}+\eta C_{D c} \frac{A_{p}}{A} \sin ^{2} \alpha
$$

The first term is based on a potential analysis and we will call it the potential term. The second term is based on a cross-flow analysis and we will call it the cross-flow term. 
The normal force is calculated as

$$
\begin{aligned}
N & =\frac{1}{2} \rho \cdot V^{2} C_{N} \\
& =\frac{1}{2} \rho V^{\prime 2}\left[\left(k_{2}-k_{1}\right) S_{h} \sin (2 \alpha) \cos \frac{\alpha}{2}+\eta C_{N_{h}} A_{r} \sin ^{2} \alpha\right]
\end{aligned}
$$

Note that by definition $\alpha$ is always positive, otherwise the cross-flow term would need to take this into account in the $\sin ^{2} \alpha$ term.

We now discretize the hull into segments (we will call the segments, panels. See Figure 2.9). The differential normal force on each panel becomes

$$
\mathrm{d} N=\frac{1}{2} \rho V^{2}\left[\left(k_{2}-k_{1}\right) \sin (2 \alpha) \cos \frac{\alpha}{2} \frac{\mathrm{d} A}{\mathrm{~d} \varepsilon} \mathrm{d} \varepsilon+\eta C_{D C} \sin ^{2} \alpha\left(2 R_{\varepsilon}\right) \mathrm{d} \varepsilon\right]
$$

where $V$ is the magnitude of the local velocity at the segment and $\mathrm{d} \varepsilon$ is the differential distance along the longitudinal axis of the hull ( $\varepsilon$ is the distance from the nose to the panel).

The normal force on each panel can be separated into potential and cross-flow terms, designated by $p$ and $c$ subscripts, respectively, and transformed into the body-fixed frame. Additionally an inertial term, designated by the subscript, $I$, will contribute to the total forces in the body-fixed frame. Thus by summing the potential, cross-flow, and inertial terms from each panel along the length of the vehicle, we can obtain the total forces in the body-fixed $Y$ and $Z$-axes.

$$
Y_{H u l l}=\sum_{1}^{n}\left[\left(Y_{p}\right)_{1}+\left(Y_{c}\right)_{i}+\left(Y_{I}\right)_{i}\right]
$$




$$
Z_{H u l l}=\sum_{1}^{n}\left[\left(Z_{r}\right)_{1}+\left(Z_{c}\right)_{1}+\left(Z_{l}\right)\right]
$$

As the axial force is independent of position along the longitudinal axis, it does not need to be broken into panels and can be expressed as a sum force on the vehicle:

$$
X_{H u l l}=-\frac{1}{2} \rho V_{c m}^{r 2} C_{H \cdot(a=1)} A \cos ^{2} \alpha
$$

We now separate the potential and cross-flow terms on each panel in the body-fixed frame:

$$
\begin{aligned}
& \mathrm{d} Z_{p}+\mathrm{d} Z_{c}=-\cos \Phi \mathrm{d} N \\
& \mathrm{~d} Y_{p}+\mathrm{d} Y_{c}=-\sin \Phi \mathrm{d} N
\end{aligned}
$$

$\Phi$ is the angle between the normal force and the body-fixed negative Z-axis, as was illustrated in Figure 2.7. We will expand the forces in the $Z$ direction first, and then expand the forces in the $Y$ direction. The potential and cross-flow terms in the $Z$ direction are

$$
\begin{aligned}
& \mathrm{d} Z_{p}=-\frac{1}{2}\left(k_{3}-k_{1}\right) \rho V^{2} \sin (2 \alpha) \cos \frac{\alpha}{2} \frac{\mathrm{d} A}{\mathrm{~d} \varepsilon} \mathrm{d} \varepsilon \cos \Phi \\
& \mathrm{d} Z_{c}=-\frac{1}{2} \rho V^{2} \eta C_{D c} \sin ^{2} \alpha\left(2 R_{\varepsilon}\right) \mathrm{d} \varepsilon \cos \Phi
\end{aligned}
$$

The added mass term, $\left(k_{3}-k_{l}\right)$, in the potential term follows the method determined by Munk, described in Section 2.2.1.1. Munk's [9] description of the 3 'main axes' 
represent the body-fixed $\mathrm{X}, \mathrm{Y}$, and $\mathrm{Z}$-axes. Thus in the $\mathrm{Z}$ direction, the added mass term will be $\left(k_{3}-k_{1}\right)$, and in the $\mathrm{Y}$ direction the added mass term will be $\left(k_{2}-k_{1}\right)$.

The following identities will be useful to us in our derivation:

$$
\begin{aligned}
& l_{1} \sin \alpha_{1}=\sqrt{w_{1}^{2}+v_{1}^{2}} \\
& l_{1} \cos \alpha_{1}=u_{1} \\
& r_{1} \sin \alpha_{1} \cos \Phi_{1}=w_{1} \\
& V_{1} \sin \alpha_{1} \sin \Phi_{1}=v_{1} \\
& \sin (2 \alpha)=2 \sin \alpha \cos \alpha \\
& \cos \left(\frac{\alpha_{1}}{2}\right)=\sqrt{\frac{V_{1}+u_{1}}{2 V_{1}}}
\end{aligned}
$$

Using these definitions we can reduce the potential term to the following:

$$
\begin{aligned}
\mathrm{d} Z_{p} & =-\frac{1}{2}\left(k_{3}-k_{1}\right) \rho V^{2} \sin (2 \alpha) \cos \frac{\alpha}{2} \frac{\mathrm{d} A}{\mathrm{~d} \varepsilon} \mathrm{d} \varepsilon \cos \Phi \\
& =-\frac{1}{2}\left(k_{3}-k_{1}\right) \rho V^{2}(2 \sin \alpha \cos \alpha) \sqrt{\frac{V+u}{2 V}} \frac{\mathrm{d} A}{\mathrm{~d} \varepsilon} \mathrm{d} \varepsilon \cos \Phi \\
& =-\left(k_{3}-k_{1}\right) \rho u w \sqrt{\frac{V+u}{2 V}} \frac{\mathrm{d} A}{\mathrm{~d} \varepsilon} \mathrm{d} \varepsilon
\end{aligned}
$$

Integrating, we find that for a small panel approximation,

$$
\begin{aligned}
\left(Z_{p}\right)_{i} & =-\left(k_{3}-k_{1}\right) \rho \int_{i}^{l+1} u_{i} w_{i} \sqrt{\frac{V_{i}+u_{i}}{2 V_{i}}} \frac{\mathrm{d} A}{\mathrm{~d} \varepsilon} \mathrm{d} \varepsilon \\
& \approx-\left(k_{3}-k_{1}\right) \rho \sqrt{\frac{V_{i}+u_{i}}{2 V_{i}}} \int_{l_{i}^{\prime+1}}^{u_{i} w_{i}} \frac{\mathrm{d} A}{\mathrm{~d} \varepsilon} \mathrm{d} \varepsilon
\end{aligned}
$$


The longitudinal location of the panel from the center of mass is defined as

$$
x_{,}=l_{0}-\varepsilon
$$

where $l_{0}$ is the distance from the nose to the center of mass. Performing the integration of the potential force term, we find that

$$
\begin{aligned}
& \left(Z_{p}\right)_{i}=-\left(k_{3}-k_{1}\right) \rho u_{1} \sqrt{\frac{V_{i}+u_{i}}{2 V_{1}}} \int_{1}^{\prime+1}\left[w+p y_{1}-q\left(l_{0}-\varepsilon\right)\right] \frac{\mathrm{d} A}{\mathrm{~d} \varepsilon} \mathrm{d} \varepsilon \\
& =-\left(k_{3}-k_{1}\right) \rho u_{i} \sqrt{\frac{V_{1}+u_{i}}{2 V_{1}}}\left\{\left[w+p y_{i}-q l_{0}\right] \int_{1}^{l+1} \frac{\mathrm{d} A}{\mathrm{~d} \varepsilon} \mathrm{d} \varepsilon+q \int_{l_{i}^{\prime \prime 1}}^{l+1} \varepsilon \frac{\mathrm{d} A}{\mathrm{~d} \varepsilon} \mathrm{d} \varepsilon\right\}
\end{aligned}
$$

Following a similar set of operations, we can derive the cross-flow force term in the $Z$ direction:

$$
\begin{aligned}
\mathrm{d} Z_{c} & =-\frac{1}{2} \rho V^{2} \eta C_{D c} \sin ^{2} \alpha\left(2 R_{\varepsilon}\right) \mathrm{d} \varepsilon \cos \Phi \\
& =-\rho w \sqrt{w^{2}+v^{2}} \eta C_{D c} R_{\varepsilon} \mathrm{d} \varepsilon
\end{aligned}
$$

Integrating the cross-flow term and using a small panel approximation we find

$$
\begin{aligned}
\left(Z_{c}\right)_{i} & =-\rho \int_{d_{i}}^{i_{i+1}} w_{i} \sqrt{w_{i}^{2}+v_{i}^{2}} \eta C_{D c} R_{\varepsilon} \mathrm{d} \varepsilon \\
& \approx-\rho \eta C_{D c} \sqrt{1+\left(\frac{v_{i}}{w_{i}}\right)^{2}} \int_{i}^{i_{i+1}} w_{i}\left|w_{i}\right| R_{\varepsilon} \mathrm{d} \varepsilon \\
\left(Z_{c}\right)_{i} & =-\rho \eta C_{D c} \sqrt{1+\left(\frac{v_{i}}{w_{i}}\right)^{2}} \operatorname{sgn}\left(w_{i}\right) \int_{i}^{i+1}\left[w+p y_{i}-q\left(l_{0}-\varepsilon\right)\right]^{2} R_{\varepsilon} \mathrm{d} \varepsilon \\
& =-\rho \eta C_{D c} \sqrt{1+\left(\frac{v_{i}}{w_{i}}\right)^{2}} \operatorname{sgn}\left(w_{i}\right) \int_{i}^{r_{1}+1}\left[\left(w+p y_{i}\right)^{2}-\right. \\
& \left.\quad 2 q\left(w+p y_{i}\right)\left(l_{0}-\varepsilon\right)+q^{2}\left(l_{0}-\varepsilon\right)^{2}\right] R_{\varepsilon} \mathrm{d} \varepsilon
\end{aligned}
$$




$$
\begin{aligned}
& =-\rho \eta C_{1 l_{1}} \sqrt{1+\left(\frac{v_{1}}{w_{1}}\right)^{2}} \operatorname{sgn}\left(w_{l}\right)\left\{\left(w+p y_{1}\right)^{2} \int_{l_{l}}^{l_{1+1}} R_{\varepsilon} \mathrm{d} \varepsilon-\right. \\
& 2 q\left(w+p y_{1}\right)\left(l_{0} \int_{l_{1}}^{l_{1}+1} R_{\varepsilon} \mathrm{d} \varepsilon-\int_{1}^{l_{1+1}} \varepsilon R_{\varepsilon} \mathrm{d} \varepsilon\right)+ \\
& \left.q^{2}\left[l_{0}^{2} \int_{l_{1}}^{l_{1}+1} R_{\varepsilon} \mathrm{d} \varepsilon-2 l_{0} \int_{l_{1}}^{l_{1+1}} \varepsilon R_{\varepsilon} \mathrm{d} \varepsilon+\int_{l_{1}}^{l_{1+1}} \varepsilon^{2} R_{\varepsilon} \mathrm{d} \varepsilon\right]\right\}
\end{aligned}
$$

By defining constants based on the geometry of the vehicle, we can simplify this equation with the following:

$$
\begin{aligned}
& I_{1 i}=\int_{1,}^{1+1} \frac{\mathrm{d} A}{\mathrm{~d} \varepsilon} \mathrm{d} \varepsilon \\
& I_{2,}=\int_{1,1}^{d_{1}} A \mathrm{~d} \varepsilon \\
& I_{3 \imath}=\int_{l_{1}}^{l_{1}+1} \varepsilon \frac{\mathrm{d} A}{\mathrm{~d} \varepsilon} \mathrm{d} \varepsilon \\
& I_{4,}=\int_{l,}^{d_{1}+1} \varepsilon A \mathrm{~d} \varepsilon \\
& I_{5 i}=\int_{l_{i}}^{i_{1+1}} \varepsilon^{2} \frac{\mathrm{d} A}{\mathrm{~d} \varepsilon} \mathrm{d} \varepsilon \\
& I_{6 i}=\int_{1}^{d_{1}+1} \varepsilon^{2} A \mathrm{~d} \varepsilon \\
& J_{1,}=\int_{t_{1}}^{t_{1+1}} 2 R_{\varepsilon} \mathrm{d} \varepsilon \\
& J_{21}=\int_{d_{1}}^{d_{1+1}} 2 R_{\varepsilon} \varepsilon \mathrm{d} \varepsilon \\
& J_{3 t}=\int_{d_{1}+1}^{i_{1}} 2 R_{\varepsilon} \varepsilon^{2} \mathrm{~d} \varepsilon \\
& J_{4 i}=\int_{i}^{1+1} 2 R_{\varepsilon} \varepsilon^{3} \mathrm{~d} \varepsilon
\end{aligned}
$$




$$
\begin{aligned}
& F_{1 i}=l_{0} I_{2 i}-I_{4 i} \\
& F_{2 i}=l_{0}^{2} I_{2 t}-2 l_{0} I_{4 t}+I_{6 t} \\
& G_{\mathrm{t}}=l_{0} I_{1,}-I_{3} \\
& G_{2 t}=l_{0}^{2} I_{1,}-2 l_{0} I_{3 i}+I_{5 i} \\
& H_{11}=l_{0} J_{1 i}-J_{21} \\
& H_{21}=l_{0}{ }^{2} J_{1},-2 l_{0} J_{2,}+J_{3 t} \\
& H_{3 \prime}=l_{0}{ }^{3} J_{11}-3 l_{0}{ }^{2} J_{2 i}+3 l_{0} J_{31}+J_{4}
\end{aligned}
$$

Thus the potential and cross-flow forces in the body-fixed Z-axis become:

$$
\begin{aligned}
& \left(Z_{p}\right)_{i}=-\left(k_{3}-k_{1}\right) \rho u_{i} \sqrt{\frac{V_{1}+u_{i}}{2 V_{i}}}\left[\left(w+p y_{i}\right) I_{1,}-G_{1}, q\right] \\
& \left(Z_{c}\right)_{i}=-\frac{\rho \eta C_{D_{c}}}{2} \sqrt{1+\left(\frac{v_{1}}{w_{1}}\right)^{2}} \operatorname{sgn}\left(w_{i}\right)\left[\left(w+p y_{i}\right)^{2} J_{1 i}-2\left(w+p y_{l}\right) H_{1} q+H_{2 i} q^{2}\right]
\end{aligned}
$$

Jones and DeLaurier [18] describe an additional normal force acting on the hull arising from inertia due to the accelerations and rotations of the hull, which is added to the potential and cross-flow forces. We will call this component the inertia term, and is described as follows:

$$
\mathbf{d F}_{\mathbf{1}}=-\left\{\left[\begin{array}{c}
\dot{u} \\
\dot{v} \\
\dot{w}
\end{array}\right] \cdot\left[\begin{array}{l}
k_{1}^{\prime} \\
k_{2}^{\prime} \\
k_{3}^{\prime}
\end{array}\right]+\left[\begin{array}{l}
p \\
q \\
r
\end{array}\right] \times\left(\left[\begin{array}{c}
u \\
v \\
w
\end{array}\right] \cdot\left[\begin{array}{c}
k_{1}^{\prime} \\
k_{2}^{\prime} \\
k_{3}^{\prime}
\end{array}\right]\right)\right\} \rho A d \varepsilon
$$

The time rate of change of the relative velocity in the body frame is calculated as

$$
\dot{\mathbf{v}}=\dot{\mathbf{v}}_{\mathrm{cm}}+\dot{\boldsymbol{\omega}} \times \mathbf{r}+\boldsymbol{\omega} \times \dot{\mathbf{r}}
$$


The last term is the convective acceleration that arises due to the fact that the fluid motion is Eulerian. Jones and DeLaurier [18] state that $\mathbf{r}$ must be regarded as the radius vector of a fluid particle that moves relative to the body frame and coincides with a fixed point at the instant considered. Thus in terms of the body motion relative to the fluid,

$$
\dot{\mathbf{r}}=-\mathbf{v}
$$

Hence,

$$
\left[\begin{array}{c}
\dot{u} \\
\dot{v} \\
\dot{w}
\end{array}\right]=\left[\begin{array}{c}
\dot{u}_{c m}+\dot{q} z-\dot{y}-q w+r v \\
\dot{v}_{c m}-\dot{p} z+\dot{r} x+p w-r u \\
\dot{w}_{c m}+\dot{p} y-\dot{q} x-p v+q u
\end{array}\right]
$$

Thus the inertia term in the body fixed $Z$ direction can be derived:

$$
\begin{aligned}
\mathrm{d} Z_{I} & =-\left(\dot{w} k_{3}^{\prime}+p v k_{2}^{\prime}-q u k_{1}^{\prime}\right) \rho A \mathrm{~d} \varepsilon \\
& =-\left[\left(\dot{w}_{c m}+\dot{p} y-\dot{q} x-p v+q u\right) k_{3}^{\prime}+p v k_{2}^{\prime}-q u k_{1}^{\prime}\right] \rho A \mathrm{~d} \varepsilon \\
& =-\left[\left(\dot{w}_{c m}+\dot{p} y-\dot{q} x\right) k_{3}^{\prime}+p v\left(k_{2}^{\prime}-k_{3}^{\prime}\right)+q u\left(k_{3}^{\prime}-k_{1}^{\prime}\right)\right] \rho A \mathrm{~d} \varepsilon
\end{aligned}
$$

Due to symmetry, $k_{2}=k_{3}$ and $k_{2}^{\prime}=k_{3}^{\prime}$. Also from Jones and DeLaurier [18],

$$
k_{3}^{\prime}-k_{1}^{\prime}=\left(k_{3}-k_{1}\right) \eta_{k}
$$

where $\eta_{k}$ is an efficiency factor accounting for the effect of the fins on the hull. We will assume that $\eta_{k}$ is equal to unity for our formulation, as generally the size of the fins are small compared to the size of the hull for AUVs. The apparent mass factors, $k_{i}$ and $k_{i}$,' are listed by Munk [9].

Thus the inertia term in the body-fixed $Z$-axis can be simplified to: 


$$
\mathrm{d} Z_{l}=-\left[\left(\dot{w}_{c m}+\dot{p} y-\dot{q} x\right) k_{3}^{\prime}+q u\left(k_{3}-k_{1}\right)\right] \rho A \mathrm{~d} \varepsilon
$$

Integrating, we can find the inertial force on each panel,

$$
\begin{aligned}
& \left(Z_{1}\right)_{1}=-\left[\left(\dot{w}_{c m}+\dot{p} y_{1}\right) k_{3}^{\prime}+q u_{1}\left(k_{3}-k_{1}\right)\right] \rho \int_{1}^{\prime \prime \prime \prime} A \mathrm{~d} \varepsilon+\rho k_{3}^{\prime} \dot{q}\left(l_{0} \int_{1,}^{\prime \prime \prime} A \mathrm{~d} \varepsilon-\int_{l_{1}^{\prime \prime 1}}^{l_{1}} \varepsilon A \mathrm{~d} \varepsilon\right) \\
& =-\left[\left(\dot{w}_{c m}+\dot{p} y_{1}\right) k_{3}^{\prime}+q u_{1}\left(k_{3}-k_{1}\right)\right] \rho I_{21}+\rho k_{3}^{\prime} \dot{q} F_{1}
\end{aligned}
$$

We can follow a similar method in the derivation of the potential, cross-flow, and inertial forces in the body-fixed $\mathrm{Y}$ direction:

$$
\begin{aligned}
\mathrm{d} Y_{r} & =-\frac{1}{2} \rho\left(k_{2}-k_{1}\right) V^{2} \sin (2 \alpha) \cos \frac{\alpha}{2} \frac{\mathrm{d} A}{\mathrm{~d} \varepsilon} \mathrm{d} \varepsilon \sin \Phi \\
& =-\frac{1}{2} \rho\left(k_{2}-k_{1}\right) V^{2}(2 \sin \alpha \cos \alpha) \sqrt{\frac{V+u}{2 V}} \frac{\mathrm{d} A}{\mathrm{~d} \varepsilon} \mathrm{d} \varepsilon \sin \Phi \\
& =-\rho\left(k_{2}-k_{1}\right) u v \sqrt{\frac{V+u}{2 V}} \frac{\mathrm{d} A}{\mathrm{~d} \varepsilon} \mathrm{d} \varepsilon
\end{aligned}
$$

Integrating and using a small panel approximation,

$$
\begin{aligned}
\left(Y_{p}\right)_{i} & =-\rho\left(k_{2}-k_{1}\right) \int_{i}^{i+1} u_{i} v_{i} \sqrt{\frac{V_{1}+u_{i}}{2 V_{i}}} \frac{\mathrm{d} A}{\mathrm{~d} \varepsilon} \mathrm{d} \varepsilon \\
& \approx-\rho\left(k_{2}-k_{1}\right) \sqrt{\frac{V_{i}+u_{i}}{2 V_{i}} \int_{i}^{\prime+1} u_{i} v_{i} \frac{\mathrm{d} A}{\mathrm{~d} \varepsilon} \mathrm{d} \varepsilon} \\
& =-\rho\left(k_{2}-k_{1}\right) u_{i} \sqrt{\frac{V_{i}+u_{i}}{2 V_{i}}} \int_{i+1}^{i+1}\left(v+r\left(l_{0}-\varepsilon\right)-p z_{i}\right) \frac{\mathrm{d} A}{\mathrm{~d} \varepsilon} \mathrm{d} \varepsilon \\
& =-\rho\left(k_{2}-k_{1}\right) u_{i} \sqrt{\frac{V_{i}+u_{i}}{2 V_{i}}}\left[\left(v+r l_{0}-p z_{i}\right) I_{1 i}-r I_{3 i}\right] \\
\mathrm{d} Y_{c} & =-\frac{1}{2} \rho V^{2} \eta C_{D c} \sin ^{2} \alpha\left(2 R_{\varepsilon}\right) \mathrm{d} \varepsilon \sin \Phi \\
& =-\rho v \sqrt{w^{2}+v^{2}} \eta C_{D c} R_{\varepsilon} \mathrm{d} \varepsilon
\end{aligned}
$$




$$
\begin{aligned}
& \left(Y_{c}\right)_{1}=-\rho \int_{l_{1}}^{l_{1+1}} v_{1} \sqrt{w_{1}{ }^{2}+v_{i}{ }^{2}} \eta C_{b_{c}} R_{\varepsilon} \mathrm{d} \varepsilon \\
& \approx-\rho \eta C_{D c} \sqrt{1+\left(\frac{v_{1}}{w_{1}}\right)^{2}} \int_{l_{1}}^{s_{1}+1} v_{1} w_{1} R_{\varepsilon} \mathrm{d} \varepsilon \\
& =-\rho \eta C_{i c_{c}} \sqrt{1+\left(\frac{v_{1}}{w_{1}}\right)^{2}} \int_{l_{1}}^{l_{1+1}}\left(v+r\left(l_{0}-\varepsilon\right)-p z_{1}\right)\left[w+p y_{1}-q\left(l_{0}-\varepsilon\right)\right] R_{\varepsilon} \mathrm{d} \varepsilon \\
& =-\frac{\rho \eta C_{D c_{c}}}{2} \sqrt{1+\left(\frac{v_{1}}{w_{1}}\right)^{2}}\left[\left(v-p z_{1}\right)\left(w+p y_{1}\right) J_{11}-q\left(v-p z_{1}\right) H_{1,}+\right. \\
& \left.r\left(w+p y_{i}\right) H_{1}-r q H_{2 i}\right]
\end{aligned}
$$

Thus

$$
\begin{gathered}
\left(Y_{r}\right)_{1}=-\rho\left(k_{2}-k_{1}\right) u_{1} \sqrt{\frac{V_{1}+u_{1}}{2 V_{1}}}\left[\left(v-p z_{1}\right) I_{1,}-r G_{11}\right] \\
\left(Y_{c}\right)_{1}=-\frac{\rho \eta C_{D_{c}}}{2} \sqrt{1+\left(\frac{v_{i}}{w_{1}}\right)^{2}}\left\{\left(v-p z_{1}\right)\left[\left(w+p y_{i}\right) J_{1,}-q H_{1,}\right]+\right. \\
\left.\quad r\left[\left(w+p y_{i}\right) H_{1,}-q H_{2 i}\right]\right\}
\end{gathered}
$$

The inertia term in the body-fixed Y direction becomes:

$$
\begin{aligned}
\mathrm{d} Y_{I} & =-\left(\dot{v} k_{2}^{\prime}-p w k_{3}^{\prime}+r u k_{1}^{\prime}\right) \rho A \mathrm{~d} \varepsilon \\
& =-\left[\left(\dot{v}_{c m}-\dot{p} z+\dot{r} x+p w-r u\right) k_{2}^{\prime}-p w k_{3}^{\prime}+r u k_{1}^{\prime}\right] \rho A \mathrm{~d} \varepsilon \\
& =-\left[\left(\dot{v}_{c m}-\dot{p} z+\dot{r} x\right) k_{2}^{\prime}+p w\left(k_{2}^{\prime}-k_{3}^{\prime}\right)+r u\left(k_{1}^{\prime}-k_{2}^{\prime}\right)\right] \rho A \mathrm{~d} \varepsilon \\
& =-\left[\left(\dot{v}_{c m}-\dot{p} z+\dot{r} x\right) k_{2}^{\prime}-r u\left(k_{2}-k_{1}\right)\right] \rho A \mathrm{~d} \varepsilon
\end{aligned}
$$

Integrating, we can find the inertial force on each panel, 


$$
\begin{aligned}
\left(Y_{1}\right)_{1} & =-\left[\left(\dot{v}_{c m}-\dot{p} z_{1}\right) k_{2}^{\prime}-r u_{1}\left(k_{2}-k_{1}\right)\right] \rho \int_{l_{1}}^{l_{1+1}} A \mathrm{~d} \varepsilon+\rho k_{2}^{\prime} \dot{r}\left(l_{0} \int_{l_{1}}^{l_{1+1}} A \mathrm{~d} \varepsilon-\int_{l_{1}}^{l_{1+1}} \varepsilon A \mathrm{~d} \varepsilon\right) \\
& =-\left[\left(\dot{v}_{c m}-\dot{p} z_{1}\right) k_{2}^{\prime}-r u_{1}\left(k_{2}-k_{1}\right)\right] \rho I_{2,}+\rho k_{2}^{\prime} \dot{r} F_{l_{1}}
\end{aligned}
$$

The pitching moments due to the normal forces on the hull for a single panel are obtained from

$$
\begin{aligned}
& \left(M_{y}\right)_{1}=-\int_{l_{1}}^{l_{1}+1}\left(l_{0}-\varepsilon\right)\left[\mathrm{d}\left(Z_{p}\right)_{i}+\mathrm{d}\left(Z_{c}\right)_{1}+\mathrm{d}\left(Z_{I}\right)_{l}\right] \\
& \left(M_{z}\right)_{l}=\int_{l_{1}+1}^{l_{1}}\left(l_{0}-\varepsilon\right)\left[\mathrm{d}\left(Y_{p}\right)_{l}+\mathrm{d}\left(Y_{c}\right)_{l}+\mathrm{d}\left(Y_{l}\right)_{l}\right]
\end{aligned}
$$

We can divide these equations into potential, cross-flow, and inertia components based on the $\mathrm{Y}$ and $\mathrm{Z}$ respective components:

$$
\begin{aligned}
& \left(M_{y p}\right)_{1}=-\int_{l_{1}}^{l_{1+1}}\left(l_{0}-\varepsilon\right) \mathrm{d}\left(Z_{p}\right)_{1} \\
& =\left(k_{3}-k_{\mathrm{\imath}}\right) \rho u_{\imath} \sqrt{\frac{V_{1}+u_{i}}{2 V_{1}}} \int_{l_{1}}^{l_{1}}\left(l_{0}-\varepsilon\right)\left[\left(w+p y_{1}-q l_{0}\right) \frac{\mathrm{d} A}{\mathrm{~d} \varepsilon} \mathrm{d} \varepsilon+q \varepsilon \frac{\mathrm{d} A}{\mathrm{~d} \varepsilon} \mathrm{d} \varepsilon\right] \\
& =\left(k_{3}-k_{1}\right) \rho u_{i} \sqrt{\frac{V_{1}+u_{i}}{2 V}}\left[\left(w+p y_{i}\right) G_{1,}-G_{2,} q\right] \\
& \left(M_{y c}\right)_{1}=-\int_{l_{i}}^{l_{1+1}}\left(l_{0}-\varepsilon\right) \mathrm{d}\left(Z_{c}\right)_{i} \\
& =\frac{\rho \eta C_{D c}}{2} \sqrt{1+\left(\frac{v_{i}}{w_{i}}\right)^{2}} \operatorname{sgn}\left(w_{i}\right) \int_{l_{i}}^{i_{1+1}}\left(l_{0}-\varepsilon\right)\left[\left(w+p y_{1}\right)^{2}\left(2 R_{\varepsilon} \mathrm{d} \varepsilon\right)-\right. \\
& \left.2\left(w+p y_{i}\right)\left(2 l_{0} R_{\varepsilon} \mathrm{d} \varepsilon-2 \varepsilon R_{\varepsilon} \mathrm{d} \varepsilon\right) q+\left(2 l_{0}^{2} R_{\varepsilon} \mathrm{d} \varepsilon-4 l_{0} \varepsilon R_{\varepsilon} \mathrm{d} \varepsilon+2 \varepsilon^{2} R_{\varepsilon} \mathrm{d} \varepsilon\right) q^{2}\right] \\
& =\frac{\rho \eta C_{D C}}{2} \sqrt{1+\left(\frac{v_{1}}{w_{i}}\right)^{2}} \operatorname{sgn}\left(w_{1}\right)\left[\left(w+p y_{1}\right)^{2} H_{1,}-2\left(w+p y_{1}\right) H_{2 l} q+H_{31} q^{2}\right]
\end{aligned}
$$




$$
\begin{aligned}
& \left(M_{y I}\right)_{1}=-\int_{I_{1}}^{l_{1}+1}\left(l_{0}-\varepsilon\right) \mathrm{d}\left(Z_{I}\right) \\
& =\int_{l_{1}}^{i_{1}}\left(l_{0}-\varepsilon\right)\left\{\left[\left(\dot{w}_{c \cdot m}+\dot{p} y_{1}\right) k_{3}^{\prime}+q u_{1}\left(k_{3}-k_{1}\right)\right] \rho A \mathrm{~d} \varepsilon+\rho k_{3}^{\prime} \dot{q}\left(l_{0} A \mathrm{~d} \varepsilon-\varepsilon A \mathrm{~d} \varepsilon\right)\right\} \\
& =\rho\left\{k_{3}^{\prime}\left[\left(\dot{w}_{c m}+\dot{p} y_{i}\right) F_{1,}-\dot{q} F_{21}\right]+q u,\left(k_{3}-k_{1}\right) F_{12}\right\} \\
& \left(M_{z p}\right)_{1}=\int_{l_{1}}^{l_{1+1}}\left(l_{0}-\varepsilon\right) \mathrm{d}\left(Y_{p}\right)_{1} \\
& =-\rho\left(k_{2}-k_{1}\right) u_{1} \sqrt{\frac{V_{1}+u_{1}}{2 V_{1}}} \int_{l_{1}}^{l_{1}}\left(l_{0}-\varepsilon\right)\left[\left(v+r l_{0}-p z_{1}\right) \frac{\mathrm{d} A}{\mathrm{~d} \varepsilon} \mathrm{d} \varepsilon-r \varepsilon \frac{\mathrm{d} A}{\mathrm{~d} \varepsilon} \mathrm{d} \varepsilon\right] \\
& =-\rho\left(k_{2}-k_{1}\right) u_{i} \sqrt{\frac{V_{1}+u_{i}}{2 V_{i}}}\left[\left(v-p z_{1}\right) G_{1,}+r G_{2 i}\right] \\
& \left(M_{z c}\right)_{i}=\int_{l_{1}}^{l_{1+1}}\left(l_{0}-\varepsilon\right) \mathrm{d}\left(Y_{c}\right)_{1} \\
& =-\frac{\rho \eta C_{L_{i}}}{2} \sqrt{1+\left(\frac{v_{1}}{w_{1}}\right)^{2}} \int_{i_{i}}^{l_{1+1}}\left(l_{0}-\varepsilon\right)\left\{( v - p z _ { 1 } ) \left[\left(w+p y_{1}\right)\left(2 R_{\varepsilon} \mathrm{d} \varepsilon\right)-\right.\right. \\
& \left.q\left(2 l_{0} R_{\varepsilon} \mathrm{d} \varepsilon-2 \varepsilon R_{\varepsilon} \mathrm{d} \varepsilon\right)\right]+r\left[\left(w+p y_{1}\right)\left(2 l_{0} R_{\varepsilon} \mathrm{d} \varepsilon-2 \varepsilon R_{\varepsilon} \mathrm{d} \varepsilon\right)-\right. \\
& \left.\left.q\left(2 l_{0}^{2} R_{\varepsilon} \mathrm{d} \varepsilon-4 l_{0} \varepsilon R_{\varepsilon} \mathrm{d} \varepsilon+2 \varepsilon^{2} R_{\varepsilon} \mathrm{d} \varepsilon\right)\right]\right\} \\
& =-\frac{\rho \eta C_{D c}}{2} \sqrt{1+\left(\frac{v_{i}}{w_{1}}\right)^{2}}\left\{\left(v-p z_{i}\right)\left[\left(w+p y_{i}\right) H_{1 i}-q H_{2 i}\right]+\right. \\
& \left.r\left[\left(w+p y_{i}\right) H_{2 i}-q H_{3 i}\right]\right\} \\
& \left(M_{z l}\right)_{i}=\int_{l_{1}}^{l_{i+1}}\left(l_{0}-\varepsilon\right) \mathrm{d}\left(Y_{c}\right)_{i} \\
& =-\int_{l_{i}}^{i_{i}+1}\left(l_{0}-\varepsilon\right)\left\{\left[\left(\dot{v}_{c m}-\dot{p} z_{i}\right) k_{2}^{\prime}-r u_{i}\left(k_{2}-k_{1}\right)\right] \rho A \mathrm{~d} \varepsilon+\rho k_{2}^{\prime} \dot{r}\left(l_{0} A \mathrm{~d} \varepsilon-\varepsilon A \mathrm{~d} \varepsilon\right)\right\} \\
& =-\rho\left\{k_{3}^{\prime}\left[\left(\dot{v}_{c m}-\dot{p} z_{i}\right) F_{1 i}+\dot{r} F_{2 i}\right]-r u_{i}\left(k_{2}-k_{1}\right) F_{1 i}\right\}
\end{aligned}
$$

The panel moments are then summed over the length of the hull to obtain the total moments on the vehicle. Additional moments arise due to a difference between the location of the longitudinal axis and the center of mass of the vehicle: 


$$
\begin{aligned}
& \left(M_{x}\right)_{H u l l}=y_{(\gamma:} Z_{H u l l}-z_{(\gamma:} Y_{H u l l} \\
& \left(M_{y}\right)_{H u l l}=\sum_{1}^{n}\left[\left(M_{y p}\right)_{1}+\left(M_{y c}\right)_{1}+\left(M_{y}\right)_{1}\right]+z_{1 \%} X_{H u l l} \\
& \left(M_{z}\right)_{\text {Hull }}=\sum_{1}^{\prime \prime}\left[\left(M_{z p}\right)_{1}+\left(M_{z c}\right)_{1}+\left(M_{z l}\right)_{1}\right]-y_{(\ell)} X_{H u l l}
\end{aligned}
$$

